\title{
Evolution and Mass Loss of Cool Ageing Stars: a Daedalean Story
}

\section{Leen Decin ${ }^{1,2}$}

1 Institute of Astronomy, KU Leuven, Celestijnenlaan 200D, B-3001 Leuven, Belgium; email: Leen.Decin@kuleuven.be

${ }^{2}$ University of Leeds, School of Chemistry, Leeds LS2 9JT, United Kingdom

Xxxx. Xxx. Xxx. Xxx. 2020. AA:1-71

https://doi.org/10.1146/((please add article doi))

Copyright (C) 2020 by Annual Reviews. All rights reserved

\section{Keywords}

evolved stars, stellar winds, stellar evolution, clumps, binaries, astrochemistry

\section{Abstract}

The chemical enrichment of the Universe; the mass spectrum of planetary nebulae, white dwarfs and gravitational wave progenitors; the frequency distribution of Type I and II supernovae; the fate of exoplanets ... a multitude of phenomena which is highly regulated by the amounts of mass that stars expel through a powerful wind. For more than half a century, these winds of cool ageing stars have been interpreted within the common interpretive framework of 1-dimensional (1D) models. I here discuss how that framework now appears to be highly problematic.

- Current 1D mass-loss rate formulae differ by orders of magnitude, rendering contemporary stellar evolution predictions highly uncertain.

These stellar winds harbour 3D complexities which bridge 23 orders of magnitude in scale, ranging from the nanometer up to thousands of astronomical units. We need to embrace and understand these 3D spatial realities if we aim to quantify mass loss and assess its effect on stellar evolution. We therefore need to gauge

- the 3D life of molecules and solid-state aggregates: the gas-phase clusters that form the first dust seeds are not yet identified. This limits our ability to predict mass-loss rates using a self-consistent approach.

- the emergence of 3D clumps: they contribute in a non-negligible way to the mass loss, although they seem of limited importance for the wind-driving mechanism.

- the 3D lasting impact of a (hidden) companion: unrecognised binary interaction has biased previous mass-loss rate estimates towards values that are too large.

Only then will it be possible to drastically improve our predictive power of the evolutionary path in 4D (classical) spacetime of any star. 


\section{Contents}

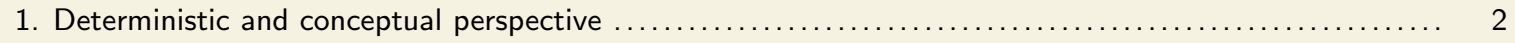

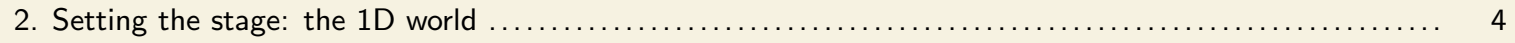

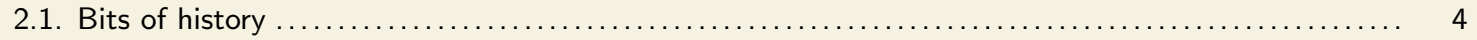

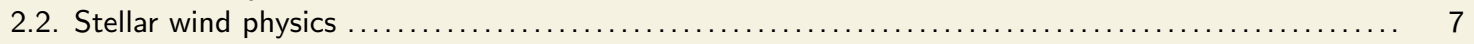

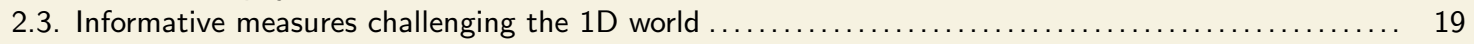

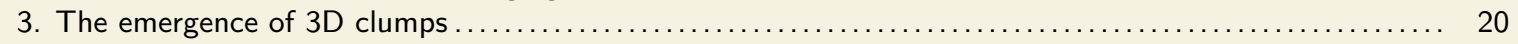

3.1. Weather map from cool ageing stars: dry with variable cloud cover $\ldots \ldots \ldots \ldots \ldots \ldots \ldots \ldots \ldots \ldots .22$

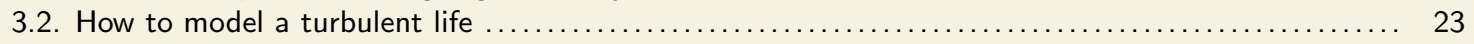

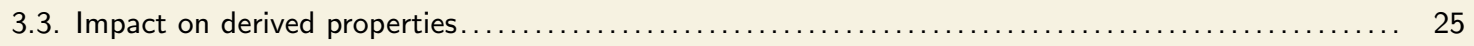

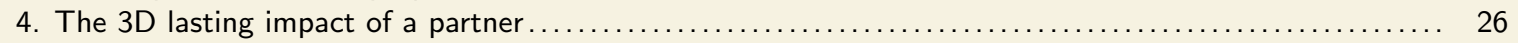

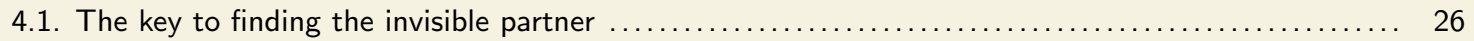

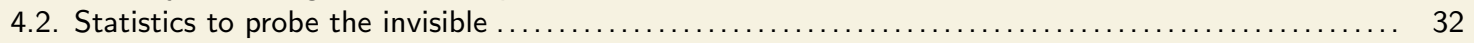

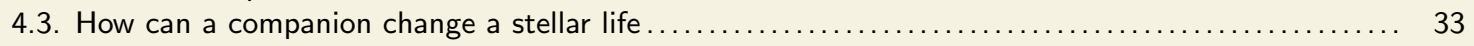

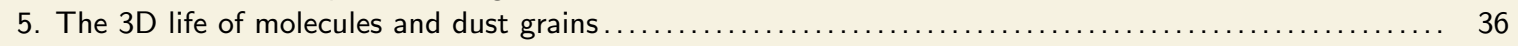

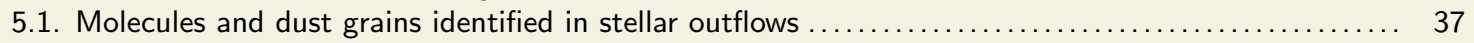

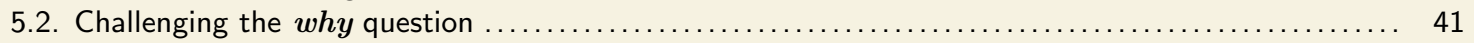

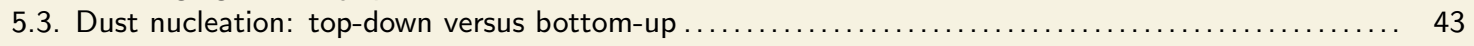

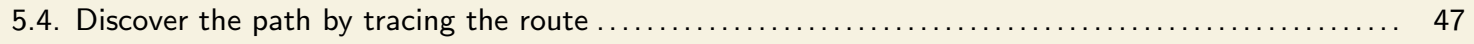

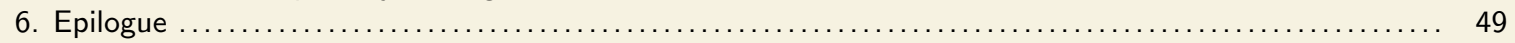

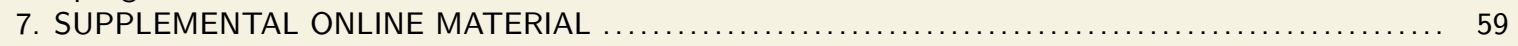

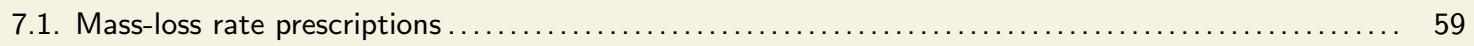

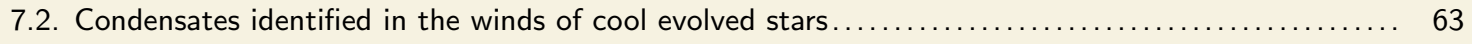

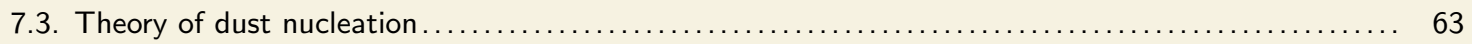

Low-and

intermediate mass

stars: stars that have an initial mass between $\sim 0.8-8 \mathrm{M}_{\odot}$ and end their life as white dwarfs if single

High-mass stars: stars with initial mass $M \gtrsim 8 \mathrm{M}_{\odot}$ that undergo core collapse at the end of their life to form a neutron star or a black hole

Stellar wind: flow of gas (and dust) particles ejected from the upper atmosphere of a star

Interstellar medium: matter and radiation that exist between the stars in a galaxy

\section{Deterministic and conceptual perspective}

Stars are born and die. Much of humankind's attention and imagination is directed towards the star-forming process, the phase during which new planets are formed around young stars that harbour the tantalising potential for new life forms to arise. Traditionally, the endphases of stellar evolution have received much less attention. Supernovae, neutron stars and black holes avoid the perception of being 'unglamorous old stars', but the research field focussing on the late stages of stellar evolution of low and intermediate mass stars is far less blessed by public excitement.

Cool ageing stars possess, however, some characteristics that turn them into key objects for both deterministic and conceptual questions in the broad field of astrophysics. The deterministic facet is linked to the chemical enrichment of our Universe. These stars are nuclear power plants that create new atoms inside their hot dense cores, including carbon, the basic building block of life here on Earth. Through their winds, they contribute 85\% of gas and $\sim 35 \%$ of dust to the total enrichment of the interstellar medium (ISM; Tielens 2005), and are the dominant suppliers of pristine building blocks of interstellar material. The deterministic aspect seeks to answer the how question — how and how much do cool ageing stars contribute to the galactic chemical enrichment? Given some initial conditions at birth, in what way can evolved stars determine the evolution of a galaxy? Key questions include: which atoms are created and in what quantity? What are the mechanisms that transport the newly created elements from the core to the outer atmosphere? Which conditions can lead to the formation of molecules and solid-state dust species? Under which circumstances 
can a stellar wind form, and what are their resulting wind velocities and mass-loss rates?

The conceptual aspect addresses the more general and overarching why question - why do cool ageing stars contribute to the galactic chemical enrichment? This question is posed in the sense of identifying the general physical and chemical laws, and their interaction. These laws are not only applicable to cool ageing stars, but to all of astrophysics. We are convinced of course that the laws of physics and chemistry are universal laws, but we must also admit that our knowledge of chemical processes is still very Earth-centric. As I will discuss below, cool ageing stars are unique laboratories which offer the exquisite possibility of teaching us about extraterrestrial, and hence universal chemistry. The how and why question inform one another and are interrelated. Ideally, we want to optimize our insight from both the deterministic and conceptual perspective so as to obtain as complete a picture as possible of the late stages of stellar evolution.

Understanding the crucial role of cool ageing stars, the Asymptotic Giant Branch (AGB) stars and their more massive counterparts the red supergiant (RSG) stars, at the level of the conceptual framework requires further explanation. Although not always recognized, these stars deserve this critical status exactly because they are thought to be 'simple'. Since the first identification of high luminosity stars by Maury in 1897, their subsequent classification as giant stars by Hertzsprung, somewhere between 1905 and 1911, and the first solid evidence of matter escaping from a red giant star by Deutsch in 1956, their atmospheric and wind structure were thought to have an overall spherical symmetry, and hence are described by one-dimensional (1D) equations. A large variety of chemical reactions occur in their wind, including unimolecular, 2- and 3-body reactions, cluster growth and grain formation. To date, more than 100 different molecules, and their isotopologues, and $\sim 15$ different solid-state species have been detected. The simple thermodynamical structure and chemically rich environment makes these stars ideal candidates for disentangling the physical and chemical processes, and unravelling the general laws governing not only these stars and their winds, but also those in other chemically rich astrophysical environments, including high-mass star-forming regions, young stellar objects, protoplanetary disks, exoplanets, novae, supernovae, and interstellar shocks.

However, recent findings complicate this picture. As I will discuss, the conceptual argument still remains valid, but its underlying reasoning gets sharpened, while essential aspects deeply grounded in the deterministic question will encounter a reformulation. Groundbreaking observations, theoretical insights, numerical simulations, and laboratory experiments bear ample evidence that our notion of spherical stars and winds was oversimplified. The cool ageing stars, that are the focus of this review, have an incredibly fascinating life and harbour 3D complexities that bridge 23 orders of magnitude in scale, from the nanometer up to thousands of astronomical units. The 3D life of molecules and solid-state aggregates, the emergence of 3D clumps, and the lasting 3D impact of a (hidden) companion offer a challenge against which our physical knowledge and chemical understanding need to be reevaluated. Just as Daedalus, these stars are a symbol of wisdom, knowledge, and power; fortunately the challenge that they pose is not a Gordian knot, but can be taken up successfully through an intensive collaboration with and access to modern observatories, state-of-the-art theoretical models, laboratory experiments, and high performance computation (HPC) facilities. Only then, will it be possible to better quantify the deterministic aspects of cool ageing stars in the cosmological context, and to have a more coherent picture of these stars for the conceptual framework.
AGB stars: cool stars, with luminosity between $\sim 2300-57500 \mathrm{~L} \odot$, that represent one of the last stages of evolution of low- to intermediate mass stars and exhibit strong stellar winds

RSG stars: cool massive stars, with luminosity of about $2000-300000 \mathrm{~L} \odot$, that evolve from stars with initial mass between $\sim 10-30 \mathrm{M}_{\odot}$ and develop powerful stellar winds

Isotopologues: molecules which differ only in their isotopic composition

Daedalus: craftsman and artist in the Greek mythology, father of Icarus, having built the paradigmatic Labyrinth for King Minos of Crete, and seen as symbol of wisdom, knowledge, and power

Gordian knot: metaphor for an intractable problem 
Circumstellar envelope: the region surrounding the star, encompassing the extended atmosphere, the stellar wind and the bow shock; see Figure 1

\section{Setting the stage: the 1D world}

Quantifying the contribution of the cool evolved AGB and RSG stars to the chemical enrichment of our Universe implies that we need to know the absolute rate by which these stars eject matter into the ISM — the mass-loss rate as a function of time, $\dot{M}(t)$ - and the relative fraction of atoms, molecules, and solid dust species in their stellar wind as functions of radial distance $r$ and time $t$. It was not until the mid seventies, that estimates of the mass-loss rate were within reach and Goldreich and Scoville presented the first detailed physical/chemical model of a circumstellar envelope (CSE) after the initial detections of circumstellar molecular line emission a few years earlier (Goldreich \& Scoville 1976). Some brief historical reminders of this enlightening period are presented in Section 2.1 Beginning with their seminal work, I introduce the reader to some basic theoretical ingredients of stellar wind physics in Section 2.2 In that section I mainly focus on the one crucial parameter of stellar evolution, which I foresee will experience major improvements in the next couple of years: the mass-loss rate during the end-phases of stellar evolution. Granted, our understanding of the relative contributions of atoms, molecules, and dust grains will see some major breakthroughs, but for the reasons I will describe below, I think that a quantitative understanding of these will take at least a decade.

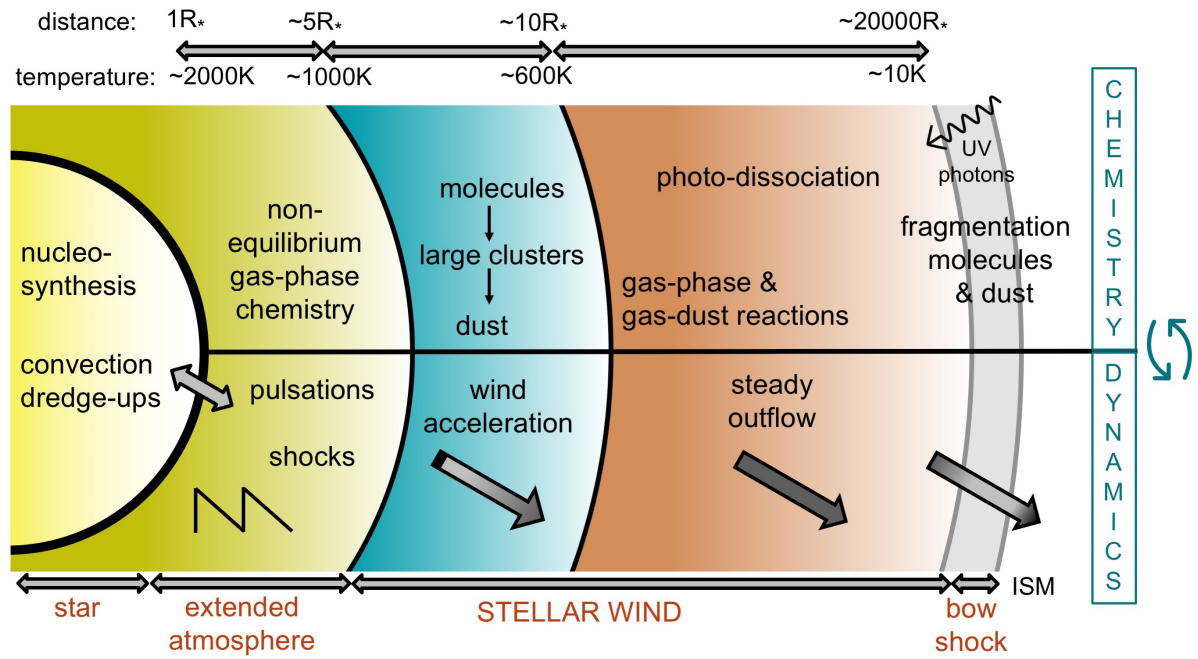

Figure 1

Schematic representation of an evolved AGB star and its circumstellar environment assuming spherical symmetry. The upper part illustrates the dominant chemical processes on length scales of nanometers to micrometers. The bottom part delineates the dynamical behaviour acting on scales of astronomical units (au). Physical and chemical processes are closely interrelated, including an intense interaction with the radiation field. Typical radial distances and temperatures are indicated at the top of the figure, where the stellar radius, $R_{\star}$, is of the order of $\sim 1 \mathrm{au}$.

\subsection{Bits of history}

The first theoretical study of dust condensation in cool stars was performed by Wildt (1933). Using thermochemical calculations, Wildt considered the possibility that dust may contribute to the opacity in cool stellar atmospheres and showed that some very refractory

4 Decin 


\section{Carbon- and oxygen-rich cool stars}

For stars in the early AGB or RSG phase, the chemical abundances reflect the chemical composition of the matter from which the star was formed. The galactic carbon-to-oxygen abundance ratio, $\mathrm{C} / \mathrm{O}$, is generally lower than 1 , with $\mathrm{C} / \mathrm{O} \sim 0.56$ in the solar vicinity and attaining lower values for lower metallicities (Chiappini et al. 2003, Akerman et al. 2004). This implies that at the start of the AGB or RSG phase, stars are still oxygen-rich $(\mathrm{O}$-rich, $\mathrm{C} / \mathrm{O}<1)$ and are categorized as M-type stars. During the AGB and RSG phase, carbon is fused in the stellar core owing to the triple- $\alpha$ process and is brought to the surface by convection. For AGB stars with an initial mass between $\sim 1.5-4 \mathrm{M}_{\odot}$ (Straniero et al. 1997), the $\mathrm{C} / \mathrm{O}$ ratio eventually becomes larger than 1 , leading to a carbon star. Due to the exceptionally high $\mathrm{C}-\mathrm{O}$ bond dissociation energy in $\mathrm{CO}$, the less abundant of the two atoms ( $\mathrm{C}$ or $\mathrm{O}$ ) is completely bound in $\mathrm{CO}$ and cannot partake in the formation of solids (Gilman 1969) and other molecules. However, recent observations have challenged this idea: molecules such as $\mathrm{CO}_{2}, \mathrm{CS}$, and $\mathrm{HCN}$ are detected in O-rich winds, and $\mathrm{H}_{2} \mathrm{O}$ and $\mathrm{SiO}$ are detected in C-rich winds (see Section 5.1).

solid compounds could be formed. But it took three decades until the far-reaching consequences of this work were realised. Motivated by the problem of identifying the origin of interstellar dust, Hoyle \& Wickramasinghe (1962) suggested that graphite grains can condense in carbon-rich cool stars and can then be driven out by radiation pressure. (See the sidebar titled Carbon- and oxygen-rich cool stars.) Meanwhile, Deutsch (1956) provided the first evidence that matter is escaping from the RSG star $\alpha$ Her: matter is flowing beyond the orbit of its $\mathrm{G}$ star companion at a speed of $\sim 10 \mathrm{~km} \mathrm{~s}^{-1}$, well above the escape velocity at that distance. The estimated mass-loss rate was around $3 \times 10^{-8}$ solar masses per year $\left(\mathrm{M}_{\odot} \mathrm{yr}^{-1}\right)$. Parker described and solved the momentum equation (see Section 2.2 for the solar wind in 1958 and introduced the term 'stellar wind' in 1960 (Parker 1958, 1960). Meanwhile, the ejection of matter was also detected for other red giants, such as the RSG star Betelgeuse (Weymann 1962b). The difficulty was explaining the apparently constant outflow of matter beyond 10 stellar radii with a speed of $\sim 10 \mathrm{~km} \mathrm{~s}^{-1}$, which is less than the escape velocity from the star (Weymann 1962a). Wickramasinghe et al. (1966) were the first to propose that radiation pressure on grains can push the grains and the gas out of the stellar gravitational potential owing to momentum exchange between the dust and the gas.

Direct evidence of late-type stars with wind mass-loss rates well above a few $10^{-8} \mathrm{M}_{\odot} \mathrm{yr}^{-1}$ came to light at the end of the $1960 \mathrm{~s}$, but before that several indirect arguments were put forward to prove that stars must lose a significant fraction of their mass during the final evolutionary phases. One such argument is due to Auer \& Woolf (1965) who found that the Hyades cluster contains about a dozen white dwarfs; each should have a mass below the Chandrasekhar limit of $1.4 \mathrm{M}_{\odot}$. But the Hyades cluster is a young group and stars with a mass of $2 \mathrm{M}_{\odot}$ are still on the main sequence. Hence, the white dwarf progenitors must have lost at least $0.6 \mathrm{M}_{\odot}$ during the post-main sequence phase, however they had not been observed at that time.

Ultimately, these mass-losing stars were detected in the late 1960s with the birth of infrared (IR) astronomy. Late M-type red giants were shown to often have an excess emission in the infrared, an effect that was attributed to circumstellar dust. In 1968/1969 Gillett
Refractory material: material resistant to decomposition by heat, pressure, or chemical attack, having a high melting point and maintaining its structural properties at high temperature

Graphite: crystalline form of the element carbon with the atoms arranged in a hexagonal structure

Late-type star: terminology used to indicate stars that are cool, here of spectral types $\mathrm{K}$ and M 


\section{Superwind}

The mass of the convective envelope decreases in time owing to both the stellar wind and nuclear burning $\left(4{ }^{1} \mathrm{H} \rightarrow 1{ }^{4} \mathrm{He}\right)$, the latter effect resulting in an increase in core mass and hence luminosity (the Paczyńskirelation; Paczyński 1970). The difference in mass between the four fusing hydrogen nuclei and the newly created helium nucleus is converted to energy according to Einstein's equation $E=m c^{2}$ (Einstein 1905). The energy production per gram by H-burning, $E_{H}$, is $6.45 \times 10^{18} \mathrm{erg} / \mathrm{g}$. It follows that the nuclear burning rate, $\dot{M}_{c}$, is given by $\dot{M}_{c}=\mathrm{L}_{\star} / E_{H}=1.02 \times 10^{-11} \mathrm{~L}_{\star} / \mathrm{L}_{\odot}\left(\right.$ in $\mathrm{M}_{\odot} \mathrm{yr}^{-1}$ ). For wind mass-loss rates above the nuclear burning rate, the associated timescale for stars to shed their envelope by a stellar wind is shorter than the nuclear burning timescale, such that mass loss determines the further evolution. Some authors denote this transition, occurring at a wind mass-loss rate of a few $10^{-7} \mathrm{M}_{\odot} \mathrm{yr}^{-1}$ (see Figure 5 in Section 2.2.4), as the superwind phase (Lagadec \& Zijlstra 2008, Zijlstra et al. 2009). However, we opt here to use the historical terminology of the word superwind, as first expressed by Renzini (1981), to indicate a mass-loss rate which greatly exceeds that prescribed by Reimers' law (see Eq. 11; Reimers 1975). We recently argued that the maximum mass-loss rate during the superwind phase is a few $10^{-5} \mathrm{M}_{\odot} \mathrm{yr}^{-1}$, and hence is around the single-scattering radiation pressure limit, indicating that the ratio of the wind momentum per second, $\dot{M} v_{\infty}$, to the photospheric radiation momentum, $L_{\star} / c$, is around 1 (Decin et al. 2019).

Maser: Microwave Amplification by Stimulated Emission of Radiation, typically visible in the micrometer and radio wavelength domain. $\mathrm{OH}$, the hydroxyl radical, is the first astronomical maser ever discovered (Weaver et al. 1965)

Planetary nebula: short $\left(\sim 10^{4}\right.$ yr $)$ evolutionary phase between the AGB and white dwarf phase; characterized by a hot central star that ionizes the gas ejected during the previous giant phase et al. (1968) and Woolf \& Ney (1969) identified silicate grains in oxygen-rich AGB and RSG stars. Gehrz \& Woolf (1971) derived the dust mass around a number of M-type stars, and using the expansion velocity from Deutsch, mass-loss rates between $10^{-7}-10^{-5} \mathrm{M}_{\odot} \mathrm{yr}^{-1}$ were obtained. The first circumstellar molecular rotational transition detected at radio wavelengths was the $\mathrm{OH}$ maser line at $1612 \mathrm{MHz}$ toward the RSG NML Cyg (Wilson \& Barrett 1968); the first thermally excited line was the CO v $=0 \mathrm{~J}=1-0$ transition detected a few years later toward the carbon star CW Leo (Solomon et al. 1971).

Using observations of similar RSG binary systems as Deutsch, Reimers (1975) was the first to derive an empirical mass-loss rate relation of the form

$$
\dot{M}=4 \times 10^{-13} \eta L / g R
$$

with $\dot{M}$ the mass-loss rate in units of $\mathrm{M}_{\odot} \mathrm{yr}^{-1}, \eta$ a unitless parameter of the order of unity, and the stellar luminosity $L$, gravity $g$, and radius $R$ in solar units. For $\eta \sim 1$, the AGB lifetime is of the order of one million years, and the maximum AGB mass-loss rate is a few $10^{-6} \mathrm{M}_{\odot} \mathrm{yr}^{-1}$ Renzini 1981). Renzini argued that a Reimers-like wind cannot explain the characteristics of planetary nebulae - the descendants of the AGB stars — and he suggested the existence of a superwind developing at the high luminosity tip of the AGB phase and with mass-loss rate of at least a few $10^{-5} \mathrm{M}_{\odot} \mathrm{yr}^{-1}$ (Renzini 1981). (See the sidebar titled Superwind.) In the same year, Glass \& Evans (1981) established the first linear relation between the $K$-band magnitude and the logarithm of the period in regularly pulsating Miratype AGB variables. (See the sidebar titled Variability and pulsation modes.) Pulsations are thought to be an essential ingredient for the wind driving in AGB stars: matter is levitated by shock waves induced by pulsations resulting in densities that are high enough at a few stellar radii for dust to condense and in sufficient momentum coupling between the gas and the grains. Observations indicate that the wind mass-loss rate ranges between $\sim 10^{-8}-10^{-4} \mathrm{M}_{\odot} \mathrm{yr}^{-1}$ and the expansion velocity between $\sim 5-30 \mathrm{~km} \mathrm{~s}^{-1}$. The discussions 


\section{Variability and pulsation modes}

Variability in brightness is a common feature of AGB stars and is mainly caused by pulsations. The classification of pulsating AGB stars into Miras, Semiregulars (SR) and Irregulars was originally based solely on the appearance of light curves, without an understanding of the physical process at work. Mira variables have regular, large amplitude variations (variation in the visible light $\delta V>2.5 \mathrm{mag}$ ); semiregular variables are of smaller amplitude $\delta V<2.5 \mathrm{mag}$ with some periodicity; and irregular variables show little periodicity although this is often due to a lack of detailed light curves. It turned out to be possible to trace the properties of variable stars through the period-luminosity $(P-L)$ diagram, in which stars form distinct sequences depending on the pulsation mode responsible for their variability (Wood et al.1999); see Fig. 1 in McDonald \& Trabucchi (2019). Pulsating stars are often multiperiodic, and normally only the period with the largest amplitude is used in the $P-L$ diagram. Mira variables are generally located on sequence $\mathrm{C}$, which is due to pulsations in the fundamental mode. Sequences B and $\mathrm{C}^{\prime}$ are due to pulsation in the first overtone mode, and sequences $\mathrm{A}$ and $\mathrm{A}^{\prime}$ to pulsation in the second and third overtone modes, respectively. Sequences D and E are, respectively, due to long secondary periods and binary stars (Wood 2015). The semiregular variables occupy sequence $\mathrm{A}$ and $\mathrm{B}$ and the lower half of sequence $\mathrm{C}$.

The energy transport, which determines the stability and growth rates of pulsation, is dominated by convection. Excitation of the pulsation modes in linear, non-adiabatic 1D models occurs through the $\mathrm{H}$ and first He ionization zones (the $\kappa$-mechanism). Most of the layers below the top of the $\mathrm{H}$ ionization zone with a temperature of $\sim 8000 \mathrm{~K}$ contribute to the determination of the period of the fundamental mode, whereas all layers (including the surface layers) contribute in determining the higher overtones modes (Fox \& Wood 1982). As a star evolves on the AGB, it rises on the $P-L$ diagram and traverses the $P-L$ sequences from left to right: specific overtone modes gradually become stable, and the primary mode shifts towards lower radial orders (Trabucchi et al. 2019, see Figure 2).

that took place during that period had a considerable impact on the field of stellar evolution modelling: in all calculations before, say, 1980 the assumption was made that the mass of a star did not change either by mass loss or mass accretion. Because the mass is the prime parameter determining the evolution and lifetime of a star, any modification to the stellar mass over time has large repercussions on its evolutionary path. A proper understanding of stellar evolution can thus not be achieved without a detailed understanding of wind mass-loss rates, and hence wind physics.

\subsection{Stellar wind physics}

A central goal of stellar wind research is to derive a relation between the mass-loss rate and fundamental stellar parameters, such as the Reimers' relation (see Eq. 1). Here I wish to address a fundamental issue in the inductive method used to derive that relation - i.e., the issue of forward versus retrieval approaches. As will become clear, the forward method tends more toward the reductionist approach in the sense that one tries to understand phenomena in terms of the interaction of the constituent parts, while the retrieval method is more inclined toward the detection of (unexpected) emergent properties and hence can be argued to be more holistic in its approach, i.e., 'the whole is more than the sum of its parts'. Both approaches are not mutually exclusive, rather they inform each other. Let me
Non-adiabatic: occurring with loss or gain of heat or mass between the thermodynamic system and its surroundings 

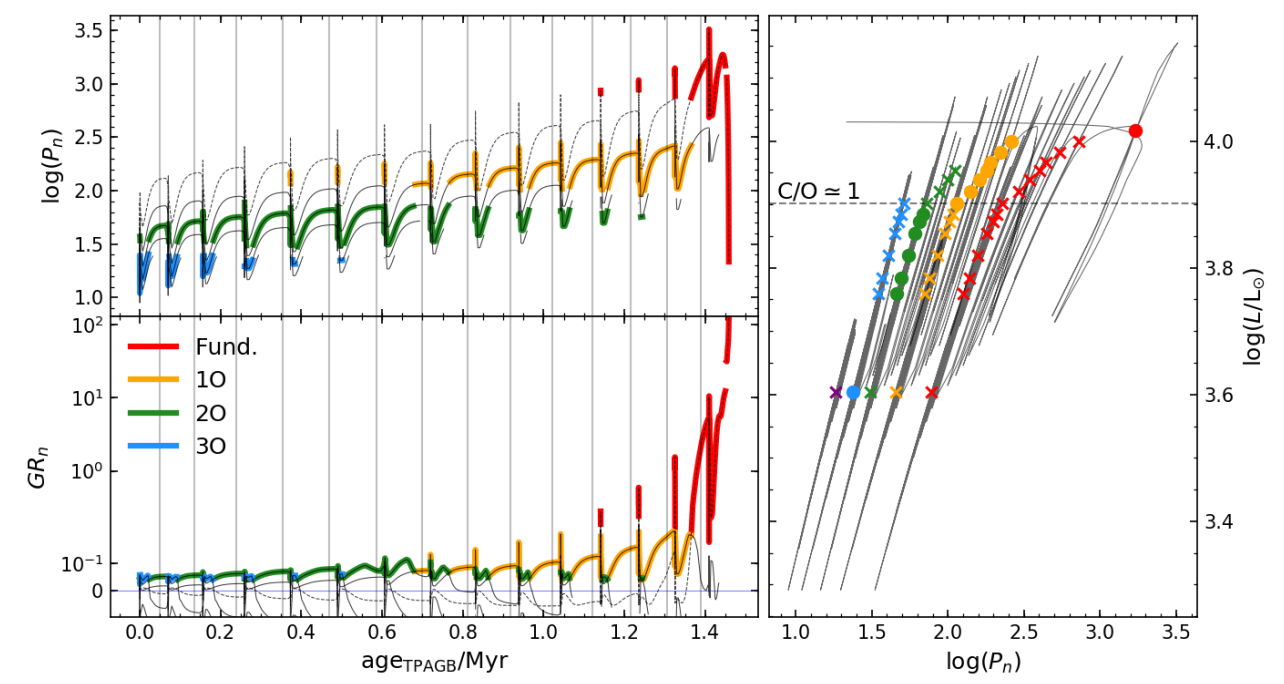

Figure 2

Pulsation periods, growth-rates, and period-luminosity diagram for a star evolving along the AGB phase. A linear, non-adiabatic $1 \mathrm{D}$ pulsation model is applied to an evolutionary track for a star with mass $M=2.6 \mathrm{M}_{\odot}$ and metallicity $Z=0.008$ at the beginning of the AGB phase. Left-hand panels: pulsation periods (top; in units of days) and growth rates (bottom) as a function of time elapsed since the beginning of thermally pulsing AGB (TP-AGB) phase. Radial pulsation modes are identified by their radial order $n$, with $n=0$ corresponding to the fundamental mode, $n=1$ to the first overtone (1O) mode, $n=2$ to the second overtone (2O) mode, and so on. For each mode $n$ with eigenfrequency $\omega_{n}=\omega_{R, n}+\mathrm{i} \omega_{I, n}$, the imaginary part of $\omega$ is the angular frequency of oscillation and the period of the $n$th mode is defined as $P_{n}=2 \pi / \omega_{I, n}$ with the amplitude growth rate calculated as $G R_{n}=\exp \left(2 \pi \frac{\omega_{R, n}}{\omega_{I, n}}\right)-1$. Dominant modes are highlighted by thick solid lines in colours. Modes other than the dominant are shown as thin solid lines, except for the fundamental mode which is shown as dashed thin lines to be more easily distinguishable. Vertical lines mark the point of maximum luminosity of quiescent evolution at each thermal pulse cycle. As time elapses, the dominant mode gradually shifts towards lower radial orders. Right-hand panel:

Radial pulsation: occurs when a star oscillates around the equilibrium state by changing its radius symmetrically over the whole surface

Thermal pulse: caused by a helium shell flash, occurring over periods of 10000 to 100000 years, lasting only a few hundred years theoretical period-luminosity diagram. Symbols correspond to quiescent evolutionary points, with the dominant mode represented by a filled circle. The quiescent evolutionary points bend towards longer periods as the luminosity increases, especially for the fundamental mode, owing to the effect of mass loss. This bending is emphasized by the models transitioning to C-rich - indicated by the horizontal grey dashed line - which causes an increase of radius with respect to O-rich models at the same luminosity. Image reproduced with permission from Trabucchi et al. (2019).

briefly describe these approaches, both in their benefits and their apparent shortcomings.

The forward approach is more mathematically oriented in that one seeks to describe all explicit and implicit relations between the quantities involved in a mathematically and physically consistent way. A self-consistent approach is even more restrictive and implies that all explicit input functions are the result of solving the system of basic equations of the problem, without introducing ad hoc assumptions. Resorting to these theoretically predicted mass-loss rates has the advantage of being able to study the explicit dependence on individual input parameters. However, the predictive power of any theoretical model is dictated by the level of description of the physical and chemical processes, and their interaction. In general, any realistic model should account for the thermodynamics, the 
hydrodynamics, the radiative transfer, and the chemistry including gas-phase and solidstate species; see Figure 3. Their combined action determines the local and global physical and chemical properties of the wind, and hence the mass-loss rate. Various interactions shown in Figure 3 are highly non-linear both with regard to the chemical and physical description, and the mathematical and numerical treatment. The forward approach can be highly demanding for the central processing unit (CPU), and hence a bottleneck for analysing large samples of observational data.

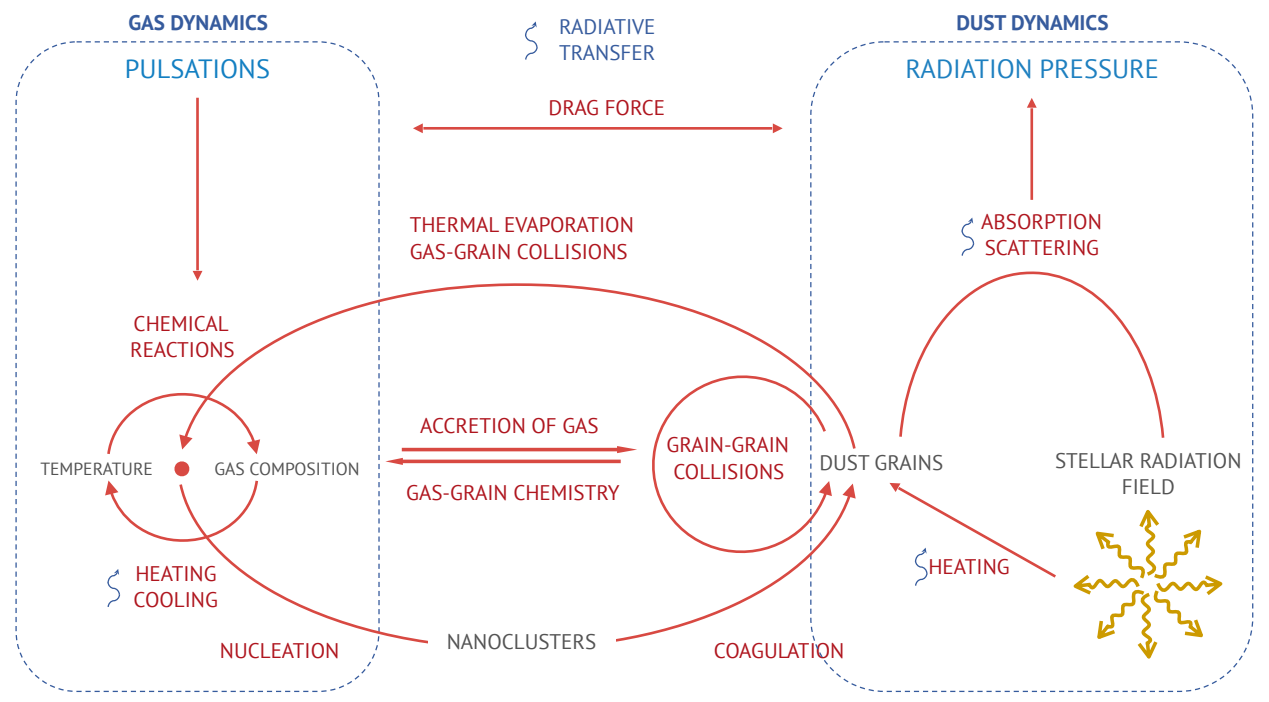

\section{Figure 3}

Schematic overview of the physical and chemical processes occurring in AGB winds. The two main driving forces are the pulsations of the star and the radiation pressure on dust grains. Both are self-consistently connected via the mechanisms depicted in the figure. Note that processes annotated with a curly blue arrow require the inclusion of radiative transfer calculations. Indicated in navy blue are wind driving forces, in black are physical and chemical quantities, in red are interactions, and in dark blue are different physics categories (dynamics and radiative transfer). This Figure is adapted from Boulangier (2019).

To model observed quantities, one often resorts to retrieval modelling. Retrieval modelling implies that one prescribes externally parameters that are basically internal parameters. These (often simplifying) parametrizations are informed by the outcome of detailed forward modelling, or by an astrophysical hypothesis informed by specific observations and their analyses. As I will demonstrate in Section 2.2.2 the solution to the momentum equation is often simplified by applying the $\beta$-velocity power law, and the solution to the energy equation by a temperature power law. The advent of new ground-based and space-borne missions has led to considerable progress in empirically derived mass-loss rate relations based on the use of retrieval methods. However, it turns out that these relations - which sometimes show dependencies on different fundamental parameters - are not always mutually consistent; see Section 2.2.4. The reasons for this discrepancy can be traced back to difficulties in determining highly accurate fundamental stellar parameters of AGB and RSG stars and the close entanglement of various of these parameters (such as luminosity, 
Spherical symmetry:

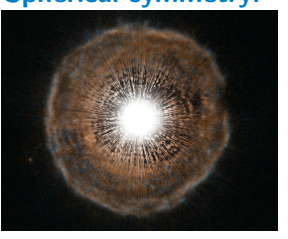

The spherical shell around the AGB star U Cam. Credit: ESA / Hubble / NASA / H. Olofsson

Drift velocity: difference between the dust and gas velocity mass, age, and pulsation period). In addition, the parametrizations inherent in the retrieval method can yield a systematic bias in the derived mass-loss rates, systematic selection effects on observed samples might induce an unrecognised bias, and it is well established that correlation does not imply causality. I will deal with some of these threats shortly.

The reductionist approach allows one to better demonstrate the various ingredients of stellar wind physics and chemistry. I therefore refer to that method in Section 2.2.1 As illustrated in Figure 1, the description of the CSE can be divided into three regions, and research groups tend to focus on the detailed description of one of them: (1) the extended atmosphere in which pulsation-induced shocks result in a chemistry that is not in thermodynamic equilibrium (e.g. Willacy \& Cherchneff 1998, Cherchneff 2006, Gobrecht et al. 2016, Höfner \& Olofsson 2018, Bladh et al. 2019), (2) the wind formation zone in which radiation pressure on newly formed dust grain leads to the onset of the stellar wind, hence initiating the mass loss (e.g. Dominik et al. 1993, Gail \& Sedlmayr 1999, Höfner \& Olofsson 2018, Bladh et al. 2019), and (3) the steady outflow zone in which the wind is freely expanding and is interacting with the surrounding interstellar radiation field resulting in photo-dissociation of molecules and further modification of the grain spectrum (e.g. Willacy \& Millar 1997, Patzer 1998, Glassgold 1999, Agúndez et al. 2010, Li et al. 2014, Van de Sande et al. 2018b). While time dependency is inherent in the description of region 1, the other two regions are often modelled using a stationary approach.

2.2.1. Standard CSE model. For our discussion, it is sufficient to remind the reader of the general conservation laws describing the stellar wind structure under the assumption of (i) stationarity, because it allows us to obtain an insight in the complex interplay between various physical and chemical processes, and (ii) spherical symmetry, because this is suggested by various observations of extended circumstellar envelopes. In this situation, the hydrodynamics as expressed in the equations of mass and momentum conservation can be written as (e.g. Goldreich \& Scoville 1976)

$$
\begin{gathered}
\frac{\mathrm{d} M(r)}{\mathrm{d} t}=\dot{M}(r)=4 \pi r^{2} \rho(r) v(r), \\
v(r) \frac{\mathrm{d} v(r)}{\mathrm{d} r}=(\Gamma(r)-1) \frac{G M_{\star}}{r^{2}},
\end{gathered}
$$

where $\dot{M}(r)$ refers to the mass-loss rate of the gas at a radial distance $r$ from the star, $\rho(r)$ is the gas density, $v(r)$ the gas velocity, $M_{\star}$ the stellar mass, $G$ the gravitational constant, and $\Gamma(r)$ the ratio of the radiation pressure force on the dust to the gravitational force which can be written as (Decin et al. 2006)

$$
\Gamma(r)=\frac{3 v(r)}{16 \pi \rho_{s} c G M_{\star} \dot{M}(r)} \iint \frac{Q_{\lambda}(a, r) L_{\lambda} \dot{M}_{d}(a, r)}{a\left[v(r)+v_{\mathrm{drift}}(a, r)\right]} \mathrm{d} \lambda \mathrm{d} a,
$$

with $\rho_{s}$ the specific density of dust, $c$ the speed of light, $\dot{M}_{d}$ mass-loss rate of the dust, $v_{\text {drift }}(a, r)$ the drift velocity of a grain of size $a, Q_{\lambda}(a, r)$ the dust extinction efficiency, and $L_{\lambda}$ the monochromatic stellar luminosity at wavelength $\lambda$.

From the first law of thermodynamics expressing the conservation of energy, the perfect gas law, and the equation of mass conservation, the thermal structure of the gas is governed by the relation (Goldreich \& Scoville 1976)

$$
\frac{1}{T(r)} \frac{\mathrm{d} T(r)}{\mathrm{d} r}=-\frac{4}{3 r}\left(1+\frac{1}{2} \frac{\mathrm{d} \ln v(r)}{\mathrm{d} \ln r}\right)+\frac{2}{3} \frac{H(r)-C(r)}{k T(r) v(r) n_{\mathrm{H}_{2}}(r)\left(1+f_{\mathrm{H}}(r)\right)}
$$


with $H(r)$ and $C(r)$ the total heating and cooling rate per unit volume, respectively, $n_{\mathrm{H}_{2}}(r)$ the number density of $\mathrm{H}_{2}$, and $f_{\mathrm{H}}(r)$ the number fraction of atomic to molecular hydrogen. The first term on the right hand side of Eq. (5) represents the cooling due to adiabatic expansion in case of constant mass loss. The second term, which represents the balance of the heating and collision-driven radiative cooling processes, needs a proper treatment of the radiative transfer and the radial abundance profile of molecules such as $\mathrm{H}_{2}, \mathrm{H}_{2} \mathrm{O}, \mathrm{CO}$ and HCN and of the grains to calculate $C(r)$ and $H(r)$; see for example Decin et al. (2010).

The balance of radiative energy gain and radiative energy loss is used to calculate the temperature of an individual grain of dust, $T_{d}$,

$$
\int_{0}^{\infty} \sigma_{d, \nu}^{\mathrm{abs}}\left[J_{\nu}(r)-B_{\nu}\left(T_{d}(a, r)\right)\right] \mathrm{d} \nu=0
$$

with $\sigma_{d, \nu}^{\text {abs }}$ the absorption opacity of the dust grain at frequency $\nu, J_{\nu}(r)$ the local mean radiation intensity, and $B_{\nu}$ the Planck function. At temperatures higher than the condensation temperature, the grain will sublimate.

For the purpose of radiation hydrodynamics, the treatment of the radiative transfer is often simplified. A well-known method has been proposed by Mihalas \& Hummer (1974) and is based on the zero- and first-order moment transport equations

$$
\begin{aligned}
& \frac{\mathrm{d} H_{\nu}\left(\tau_{\nu}\right)}{\mathrm{d} \tau_{\nu}}=J_{\nu}\left(\tau_{\nu}\right)-S_{\nu}\left(\tau_{\nu}\right) \\
& \frac{\mathrm{d} K_{\nu}\left(\tau_{\nu}\right)}{\mathrm{d} \tau_{\nu}}=H_{\nu}\left(\tau_{\nu}\right),
\end{aligned}
$$

with $\tau_{\nu}$ the optical depth, $S_{\nu}$ the source function, $H_{\nu}$ the flux, and $K_{\nu}$ being related to the radiation pressure $p_{\nu}=(4 \pi / c) K_{\nu}$. Eqs. $7-8$ is a system of coupled integro-differential equations that can be mathematically closed by defining the Eddington factor

$$
f_{\nu}(r)=\frac{K_{\nu}(r)}{J_{\nu}(r)}
$$

with $f_{\nu}(r)$ approaching $1 / 3$ for an isotropic radiation field.

The chemical evolution of the composition of a closed system is dictated by a set of chemical formation and destruction reactions. Mathematically, this is a set of coupled ordinary differential equations where the change in number density of the $i$ th species is given by

$$
\frac{\mathrm{d} n_{i}}{\mathrm{~d} t}=\sum_{j \in F_{i}}\left(k_{j} \prod_{r \in R_{j}} n_{r}\right)-\sum_{j \in D_{i}}\left(k_{j} \prod_{r \in R_{j}} n_{r}\right) .
$$

The first term, within the summation, represents the rate of formation of the $i$ th species by a single reaction $j$ of a set of formation reactions $F_{i}$. The second term is the analogue for a set of destruction reactions $D_{i}$. Each reaction $j$ has a set of reactants $R_{j}$, where $n_{r}$ is the number density of each reactant and $k_{j}$ the rate coefficient of this reaction. For chemistry in thermodynamic equilibrium, Eq. 10, involving both gaseous and dust species, reduces to the well-known law of mass action (Gail \& Sedlmayr 2013)

$$
\prod_{\substack{\mathrm{i} \\ \text { all gases }}}\left(\frac{p_{i}}{p_{0}}\right)^{\nu_{i}} \prod_{\substack{\mathrm{i} \\ \text { all solids }}}\left(a_{i}^{c}\right)^{\nu_{i}}=\mathrm{e}^{-\Delta G / R T},
$$


Stoichiometric coefficient: number written in front of each compound in a chemical reaction to balance the number of each element on both the reactant and product sides of the equation
Dust condensation radius: radial distance at which the first solid-state species are formed

Terminal wind velocity: at large distance from the star, the velocity asymptotically approaches the terminal wind velocity with $\nu_{i}$ the stoichiometric coefficients, the activity $a_{i}^{c}$ of species $i$ defined as $a_{i}^{c}=p_{i} / p, p$ the pressure, $p_{i}$ the partial pressure of species $i, p_{0}$ the standard pressure of 1 bar, $R$ the gas constant in units as used for the data of $\Delta G$, and $G$ the Gibbs function so that

$$
\Delta G=\sum_{i} \nu_{i} G_{i}\left(p_{0}, T\right)
$$

with $G_{i}(p, T)$ the partial free enthalpy of 1 mole of species $i$ at temperature $T$ and pressure $p$. Chemical equilibrium is established if the chemical reaction time scales are small compared with other competing time scales governing the considered concentrations, so that Eq. 11 . is only dependent on temperature.

Together with suitable boundary and initial conditions, the resulting mathematical system $\mathcal{M}$ described in Eqs. 2 - 12 constitutes a complete and well-posed set of coupled equations given a set of independent fundamental stellar parameters. The solution provides a theoretical prediction of the physical and chemical quantities, including the mass-loss rate and the spectral appearance. By choosing the stellar mass, temperature, luminosity, and abundance composition $\left\{M_{\star}, T_{\star}, L_{\star},\left\{\epsilon_{X}\right\}\right\}$ as independent stellar parameters, we can express this formally as (Gail \& Sedlmayr 2013)

$$
\left\{M_{\star}, T_{\star}, L_{\star},\left\{\epsilon_{X}\right\}\right\} \stackrel{\mathcal{M}}{\Longrightarrow} \dot{M}
$$

Admittedly, the chemical abundances $\left\{\epsilon_{X}\right\}$ are genuinely free parameters only for stars in the early AGB phase. In principle the elemental abundances result from stellar evolution - i.e., in particular nucleosynthesis and convection-induced dredge-up processes - so the stellar mass and chemical abundances are not independent. However, the time scales involved with stellar evolution are much larger than those of the physical and chemical processes considered here. This implies that mass and chemical abundances only vary on secular time scales and can be considered as independent stellar parameters.

2.2.2. Outcome of stationary 1D model predictions. Given the assumption of stationarity, any model prediction applies for a genuinely dust-driven wind; but see Section 2.2.3. The radial structure for a low-mass carbon-rich star at the tip of the AGB phase is shown in Figure 4 Efficient dust nucleation around 3 stellar radii enables a dust-driven wind. The gas pressure, and hence also the density $\rho$, decreases approximately exponentially near the photosphere, with $\rho \propto r^{-2}$ further out due to the condition of mass conservation. The gas expansion velocity, $v$, exceeds the local escape velocity, $v_{\text {esc }}$, around 3 stellar radii and the wind becomes gravitationally unbound reaching a terminal wind velocity, $v_{\infty}$, of around $22 \mathrm{~km} \mathrm{~s}^{-1}$. In retrieval modelling, this particular behaviour of the wind acceleration is often approximated by the so-called $\beta$-type velocity law (Lamers \& Cassinelli 1999)

$$
v(r)=v_{0}+\left(v_{\infty}-v_{0}\right)\left(1-\frac{R_{\text {dust }}}{r}\right)^{\beta},
$$

with $r$ the distance to the star and $v_{0}$ the velocity at the dust condensation radius $R_{\text {dust }}$. Low values for $\beta$ describe a situation with a high wind acceleration. In the same vein the gas and dust temperature structure are often approximated by a power law

$$
T(r)=T_{\star}\left(\frac{R_{\star}}{r}\right)^{\zeta}
$$



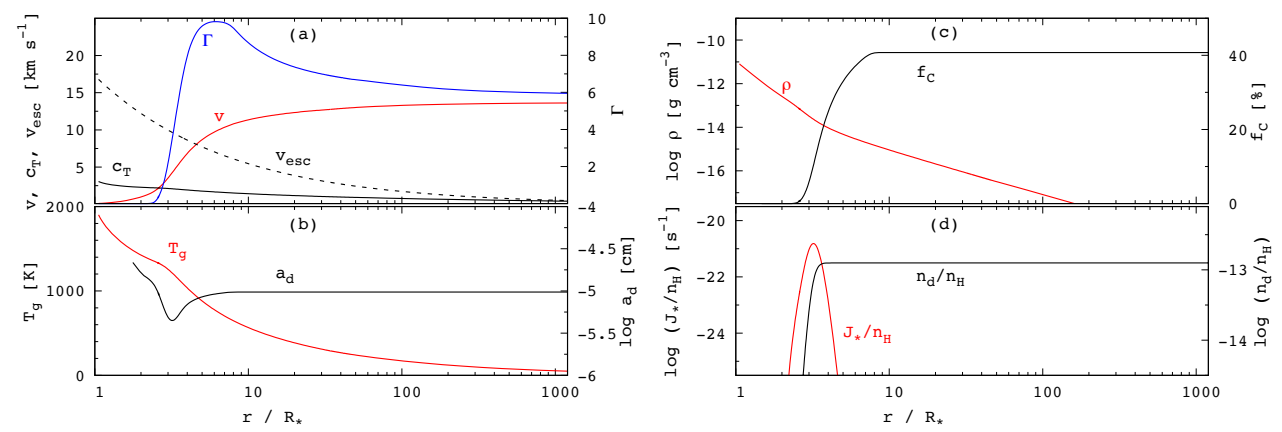

Figure 4

Radial structure for a stationary dust-driven wind with independent fundamental parameters $\mathrm{M}_{\star}=0.7 \mathrm{M}_{\odot}, \mathrm{L}_{\star}=2.4 \times 10^{4} \mathrm{~L}_{\odot}, \dot{M}=8 \times 10^{-5} \mathrm{M}_{\odot} \mathrm{yr}^{-1},\left\{\epsilon_{X \neq C}\right\}_{\odot}, \epsilon_{\mathrm{C}} / \epsilon_{\mathrm{O}}=1.4$ and assuming homogeneous nucleation with $\mathrm{C}_{1}$ as basic monomer. (a) gas velocity $v$, isothermal sound speed $c_{T}$, escape velocity $v_{\text {esc }}$, and radiative acceleration in units of the gravitational acceleration $\Gamma$;

(b) radiative equilibrium gas temperature $T_{g}$ and dust grain size $a_{d}$; (c) mass density $\rho$ and degree of condensation, that is the fraction $f_{c}$ of the amount of carbon condensate relative to the condensible carbon present in the gas phase; $(d)$ nucleation rate $J_{\star}$ and grain particle density $n_{d}$, both quantities normalized to the number density of hydrogen nuclei $n_{\mathrm{H}}$. The nucleation rate $J_{\star}$ corresponds to the formation rate of the gas-phase cluster that is the least abundant, and hence will dictate the dust formation rate; see Section 5.3 Figure courtesy H.-P. Gail.

with $\zeta \approx 0.5-0.6$.

Stationary wind models typically predict terminal wind velocities between $5-20 \mathrm{~km} \mathrm{~s}^{-1}$, and mass-loss rates between $\sim 5 \times 10^{-8}-3 \times 10^{-4} \mathrm{M}_{\odot} \mathrm{yr}^{-1}$ for carbon-rich winds, where the maximum mass-loss rate is a factor of a few lower for oxygen-rich winds (De Beck et al. 2010, Gail \& Sedlmayr 2013 Decin et al. 2019). A higher luminosity and lower temperature induce higher mass-loss rates and expansion velocities due to the larger radiation pressure on the dust grains and the potential for more efficient dust nucleation and growth, respectively. The mass-loss rate is very sensitive to the stellar mass via its effect on the gravity and hence $\Gamma(r)$ : a reduction of the stellar mass by a factor of 2 leads to an increase of the mass-loss rate by a factor 3 to 100 (Gail \& Sedlmayr 2013). This effect might hence be a natural candidate to explain the superwind scenario proposed by Renzini (1981), since the AGB stellar mass will be reduced significantly by the preceding mass loss.

For RSG stars the role of grains close to the star remains unresolved, and radiation pressure on molecular lines, turbulent pressure, acoustic waves and Alfvén waves have been proposed as alternative mechanism (Josselin \& Plez 2007, Bennett 2010, Scicluna et al. 2015. Montargès et al. 2019, Kee et al. 2020). In general, these alternative processes might also support the AGB stellar wind, although their role in driving the wind is very much debated (Wood 1990, Gustafsson \& Höfner 2003).

2.2.3. Limitations of the stationary approach. The standard 1D CSE description (Section 2.2.1 is time independent, and hence does not treat the extended atmosphere in which pulsations induce shock waves that levitate the gas to distances where dust can form. It is generally accepted that pulsations are a key ingredient of AGB mass loss (Bowen 1988, McDonald \& Zijlstra 2016, McDonald \& Trabucchi 2019, See the sidebar titled Variability and mass-loss rate), however the nature of the pulsations and their impact on the density

Turbulent pressure: pressure caused by small-scale motions of stochastic nature; $P_{\text {turb }}=\zeta \rho v_{\text {turb }}^{2}$ with $v_{\text {turb }}$ the turbulent velocity and $\zeta$ equal to 1 in the case of isotropic turbulence

Acoustic wave: mechanical and longitudinal wave resulting from $3 \mathrm{D}$ fluctuations in the pressure field

Alfvén wave: a transverse electromagnetic wave propagating along the magnetic field lines of a plasma and resulting from an interaction of the magnetic field and the electric currents within it (Alfvén 1942) 


\section{Variability and mass-loss rates}

For Mira-type variable stars with a luminosity above $\sim 2000 \mathrm{~L}_{\odot}$ and pulsation period $P$ between $\sim 300$ - 800 days, a linear relation exists between the period and the logarithm of the mass-loss rate $\left(\sim 10^{-7} \mathrm{M}_{\odot} \mathrm{yr}^{-1}<\dot{M}<3 \times 10^{-5} \mathrm{M}_{\odot} \mathrm{yr}^{-1}\right)$, suggesting that the corresponding increase in luminosity causes the radiation pressure on dust to be more effective. Semiregular variables with $P \lesssim 200$ days cover essentially the same mass-loss rate regime as the Mira variables with period between $200-400$ days, while a maximum mass-loss rate of a few $10^{-5} \mathrm{M}_{\odot} \mathrm{yr}^{-1}$ seems to be reached for $P \gtrsim 800$ days (Vassiliadis \& Wood 1993, De Beck et al. 2010). Between $\sim 60$ and $\sim 300$ days, an approximately constant mass-loss rate of $\sim 3.7 \times 10^{-7} \mathrm{M}_{\odot} \mathrm{yr}^{-1}$ is found, while for $P<60$ days the mass-loss rate is a factor of $\sim 10$ smaller. This rapid increase of mass-loss rate and dust production when the star first reaches a pulsation period of $\sim 60$ days coincides approximately with the point when the star transitions to the first overtone pulsation mode, while the second rapid mass-loss-rate enhancement at $P \sim 300$ days coincides with the transition to the fundamental pulsation mode. This indicates that stellar pulsations are the main trigger for the onset of the AGB mass loss and are significant in controlling the mass-loss rate (McDonald \& Zijlstra 2016, McDonald \& Trabucchi 2019).

Non-radial pulsation: some parts of the stellar surface are moving inwards, while others move outwards at the same time scale height continues to be a source of debate. The stellar interior where these variations originate is an optically thick region dominated by convection that has proven difficult to model. Linear, non-adiabatic 1D pulsation models have been successful in predicting the overtone modes expected to occur in early-type AGB semiregular variables, but poor agreement is found for the fundamental mode Mira-type pulsators (Trabucchi et al. 2017, 2019 ).

A global 3D radiation-hydrodynamics approach of the convection, and related pulsations, has recently been explored by Freytag et al. (2017). Due to computational constraints, the models only reach up to $\sim 2 \mathrm{R}_{\star}$. Irregular structures with convection cells dominate in the interior part and propagating shocks in the outer atmosphere. The models develop radial and non-radial pulsations, but with a different frequency between the inner and outer part of the model. For models in which the radial fundamental mode dominates, the pulsation periods range between $\sim 300-630$ days, in good agreement with observations for Mira-type variables. The exact mechanism for the mode excitation is, however, not yet fully understood, and possibilities such as stochastic excitation by convection, excitation by the $\kappa$-mechanism, and acoustic noise are explored by the authors.

Owing to their complex nature various researchers have treated the effects of pulsations in a 1D parametrized way following the piston approach (Bowen 1988, Gauger et al. 1990, Höfner et al. 1995). These wind models typically have an inner boundary situated just below the photosphere of the star where the radius and luminosity are assumed to have sinusoidal variations characterized by the pulsation period $P$ and velocity amplitude $\Delta v_{p}$. The pulsation period can be derived from the period-luminosity $(P-L)$ relation. The velocity amplitude, $\Delta v_{p}$, typically ranges between $2-4 \mathrm{~km} \mathrm{~s}^{-1}$, corresponding to shock amplitudes of $\sim 15-20 \mathrm{~km} \mathrm{~s}^{-1}$ in the inner atmosphere (Bladh et al. 2019). This standard inner boundary condition is meant to describe the pulsation properties of Mira variables pulsating in the fundamental mode, but a similar simplified approach for semiregular variables has not yet been pursued. Formally, we can express the 1D piston models as (Gail \& 


$$
\left\{M_{\star}, T_{0}, L_{0},\left\{\epsilon_{X}\right\}, P, \Delta v_{p}\right\} \stackrel{\mathcal{M}}{\Longrightarrow} \dot{M},
$$

with $T_{0}=\mathrm{T}_{\star}(t=0)$ and $L_{0}=\mathrm{L}_{\star}(t=0)$. The predicted wind velocities and mass-loss rates show no significant differences compared to more complex models in which (the mean of) the dynamical properties predicted by the 3D radiation-hydrodynamic models are used as inner boundary condition for the dust-driven wind (Liljegren et al. 2018).

Using the piston approach, model grids for carbon- and oxygen-rich winds have been published by Arndt et al. (1997), Eriksson et al. (2014) and Bladh et al. (2019), respectively. The grid of 48 models by Arndt et al. (1997) self-consistently calculates the dust nucleation by assuming chemical equilibrium $(\mathrm{CE})$ and homogeneous nucleation with $\mathrm{C}_{1}$ as the basic monomer, while the more extensive grids of Eriksson et al. (2014) and Bladh et al. (2019) assume the presence of dust seeds that can act as further building blocks for grain growth. More on these two different approaches can be found in Section 5. Arndt et al. (1997) have presented a linear multivariate regression analysis by means of a multidimensional maximum-likelihood method to derive an explicit mass-loss rate formula for the implicit mass-loss relation of pulsation-enhanced dust-driven winds, with best-fit formula being

$$
\begin{aligned}
\log \dot{M}_{\mathrm{fit}}= & -4.95-2.8 \log \left(\mathrm{M}_{\star}\left[\mathrm{M}_{\odot}\right]\right)+1.65 \log \left(\frac{L_{0}\left[\mathrm{~L}_{\odot}\right]}{10^{4}}\right)-9.45 \log \left(\frac{T_{0}[\mathrm{~K}]}{2600}\right) \\
& +0.470 \log \left(\frac{\epsilon_{\mathrm{C}} / \epsilon_{\mathrm{O}}}{1.8}\right)-0.146 \log \left(\frac{P[\text { days }]}{650}\right)+0.449 \log \left(\frac{\Delta v_{p}\left[\mathrm{~km} \mathrm{~s}^{-1}\right]}{2}\right), 17 .
\end{aligned}
$$

with $\dot{M}_{\text {fit }}$ in units of $\mathrm{M}_{\odot} \mathrm{yr}^{-1}$. From the regression coefficients it is clear that $\dot{M}_{\text {fit }}$ is strongly influenced by $T_{0}, \mathrm{M}_{\star}$, and $L_{0}$ and is only weakly dependent on $\Delta v_{p}, \epsilon_{\mathrm{C}} / \epsilon_{\mathrm{O}}$, and $P$. This outcome renders the possibility of a reduced fit, with an equally high correlation coefficient,

$$
\log \dot{M}_{\text {fit }}=-4.93-2.88 \log \left(\mathrm{M}_{\star}\left[\mathrm{M}_{\odot}\right]\right)+1.53 \log \left(\frac{L_{0}\left[\mathrm{~L}_{\odot}\right]}{10^{4}}\right)-8.26 \log \left(\frac{T_{0}[\mathrm{~K}]}{2600}\right) .
$$

The results published by Bladh et al. (2019) allow for a similar linear multivariate regression analysis, yielding

$$
\log \dot{M}_{\mathrm{fit}}=-5.26-3.82 \log \left(\mathrm{M}_{\star}\left[\mathrm{M}_{\odot}\right]\right)+3.17 \log \left(\frac{\mathrm{L}_{\star}\left[\mathrm{L}_{\odot}\right]}{10^{4}}\right)-6.47 \log \left(\frac{\mathrm{T}_{\text {eff }}[\mathrm{K}]}{2600}\right) .
$$

While Eqs. 17 - 18 refer to the temperature and luminosity at time $t=0$ where the piston position takes its mean value over the pulsation period and is moving outward with maximum speed, Eq. 19] uses the effective temperature and stellar luminosity of the hydrostatic dust-free model that was used as starting structure for the calculations. Although any impact of the pulsation practically cancels in Eqs. 18 -19, the pulsation quantities have an important implicit influence. The reason is that pulsations increase the density scale height allowing for an efficient condensation and growth of dust species. This outcome explains why empirically derived mass-loss rate formula, such as the one proposed by Reimers, can be expressed in terms of fundamental stellar parameters without notion of the pulsation characteristics; for Reimers' law being $L_{\star}, R_{\star}$, and $M_{\star}$ (see Eq. 1 in Section 2.1).

2.2.4. Theoretical versus empirical mass-loss rate relations. The similar dependence between the mass-loss rate and some of the fundamental stellar parameters identified by the
Seed particle: tiny

solid particle, predicted using nucleation theory or assumed to pre-exist and typically chosen to consist of 1000 monomers or to have a radius of $1 \mathrm{~nm}$ 
forward and the retrieval approaches has nurtured the idea that empirically derived massloss rate relations could provide an alternative approach for understanding the essence, if not the detail, of the process by which mass loss occurs. The advent of new observing facilities resulted in tremendous progress in the field of observational astrophysics. Molecular lines and dust emission have been used to retrieve the mass-loss rate of the stars under study (Höfner \& Olofsson 2018, and references therein). Circumstellar CO rotational and $\mathrm{OH}$ maser emission are the molecular diagnostics most often used to estimate the gas mass-loss rates (e.g. Baud \& Habing 1983. Schöier et al. 2002, Decin et al. 2006, Ramstedt et al. 2008, De Beck et al. 2010). The benefit of analysing molecular lines is that one obtains the expansion velocity. The disadvantages are that the observation of emission from thermally excited lines is typically limited to nearby stars within $\sim 2 \mathrm{kpc}$ from the Sun (although the sensitivity of ALMA is now opening up the field to larger distances, such as the Large Magellanic Cloud; Groenewegen et al. 2016), the unknown fractional abundance of the molecule, and the fact that the analysis often requires a non-local thermodynamic equilibrium (non-LTE) radiative transfer analysis which can be quite CPU intensive. The latter aspect implies that sample studies seldom exceed $\sim 50$ stars (Danilovich et al. 2015); whereas the calculation of dust spectral features - and the related analysis of the spectral energy distribution (SED) - are readily applied to large samples, owing to the inherently simpler radiative transfer calculations. However, the identification of dust features is more ambiguous, and a reliable estimate of the mass-loss rate of the dust can only be achieved if several dust features with differing optical depths are combined. In addition, one needs to assume a dust expansion velocity and a gas-to-dust mass ratio to convert the derived dust densities into gas mass-loss rates (e.g., Heras \& Hony 2005, Verhoelst et al. 2009, Groenewegen et al. 2009).

Supported by the incredible increase in computational power during the last two decades, a whole family of mass-loss rate relations has been derived. Without any attempt for completeness, I have summarized some of these theoretical, empirical and semi-empirical relations in the Supplemental Text, where I focus on those relations which have an explicit dependence on two of the main fundamental stellar parameters: the luminosity and the effective temperature. For an AGB star of stellar mass $2 \mathrm{M}_{\odot}$ or with an effective temperature of $2800 \mathrm{~K}$, these mass-loss rate relations are shown in the upper panels of Figure 5 . for a RSG star of mass $15 \mathrm{M}_{\odot}$ or with an effective temperature of $3500 \mathrm{~K}$, the mass-loss prescriptions are shown in the bottom panels of Figure 5 more detailed information is provided in the Supplemental Text. The large diversity between prescriptions is remarkable, with differences up to 2 orders of magnitude or higher. To be fair, not all mass-loss rate formula are applicable to all classes of cool ageing stars, so sometimes we might be comparing apples with oranges. But even within the class of the 'apples', it seems that we are having different cultivars in the same basket; let's call them the medium-sized Golden Russets which make extraordinary cider and the large red-coloured Haralsons which are an excellent choice for pies. Both are different genomic expressions of the malus pumila and in the same vein the relations we witness in Figure 5 are different expressions of an 'emergent property': the mass-loss rate. This statement deserves further explanation.

Indeed, while the various mass-loss rate proposals seem already disappointingly incompatible with huge differences, there is still another underlying conceptual complication. Given these differences, it is to be expected that the impact of a particular choice of massloss rate on stellar evolution calculations will be significant. This is illustrated in Figure 6 where a set of simplified evolutionary tracks is shown for stars with an AGB mass at the 

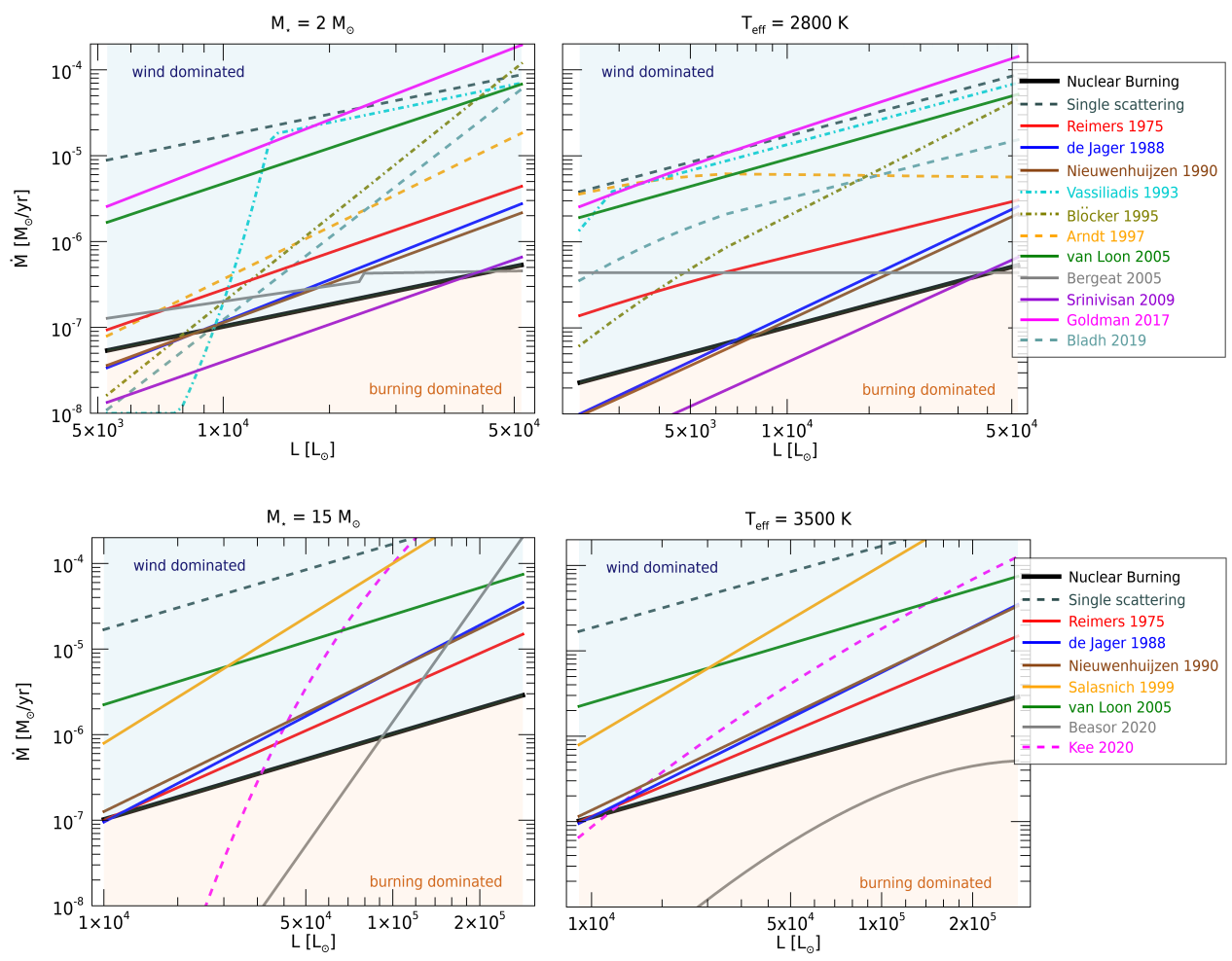

Figure 5

Mass-loss rate as function of luminosity. The upper two panels show mass-loss rate prescriptions for AGB stars, at a fixed stellar mass of $2 \mathrm{M}_{\odot}$ (left) and fixed effective temperature of $2800 \mathrm{~K}$ (right); the bottom two panels apply to RSG stars, at a fixed stellar mass of $15 \mathrm{M}_{\odot}$ (left) and fixed effective temperature of $3500 \mathrm{~K}$ (right). Empirical mass-loss rate relations are displayed with a full line, semi-empirical relations with a dash-dotted line, and theoretical relations with a dotted line. The rate at which hydrogen is consumed by nuclear burning, $\dot{M}_{c}=1.02 \times 10^{-11} \mathrm{~L}_{\star}$, is shown as thick black line; the single-scattering radiation pressure limit for an expansion velocity of $12 \mathrm{~km} \mathrm{~s}^{-1}$ is shown as dashed dark grey line. Stellar mass loss rules the evolution of the AGB and RSG stars if the wind mass-loss rate exceeds the nuclear burning rate, as indicated by the light-blue region; the hydrogen-burning dominated region is indicated by the light-orange region. The change in the slope for the Vassiliadis \& Wood (1993) description is caused by the stellar mass exceeding the limit of $2.5 \mathrm{M}_{\odot}$ (see Eqs. $27-28$ ); the jump for the Bergeat \& Chevallier (2005) description in the left panel is due to the effective temperature getting below $2900 \mathrm{~K}$ (Eqs. 37-38). More detailed information is provided in the Supplemental Text.

first thermal pulse between $0.8-3 \mathrm{M}_{\odot}$ (see the Supplemental Text for more information). For the Blöcker (1995) and Vassiliadis \& Wood (1993) mass-loss rate relations which have a strong dependence on luminosity, the stars evolve on a nearly horizontal track where the mass remains approximately constant — until the star reaches the locus where $\mathrm{d} \log \mathrm{M}_{\star} / \mathrm{d} t=\mathrm{d} \log \mathrm{L}_{\star} / \mathrm{d} t$ (referred to as the 'cliff' by Willson 2000, see filled red circles in Figure 6. For the set of models shown in Figure 6. the mass-loss rate at the 'cliff' is around $0.5-1.7 \times 10^{-6} \mathrm{M}_{\odot} \mathrm{yr}^{-1}$. Passing beyond that 'cliff' implies that the evolution is further ruled by the wind mass-loss rate, which for the Blöcker (1995) and Vassiliadis \& Wood (1993) relations implies an asymptotic downhill behaviour. This particular behaviour 

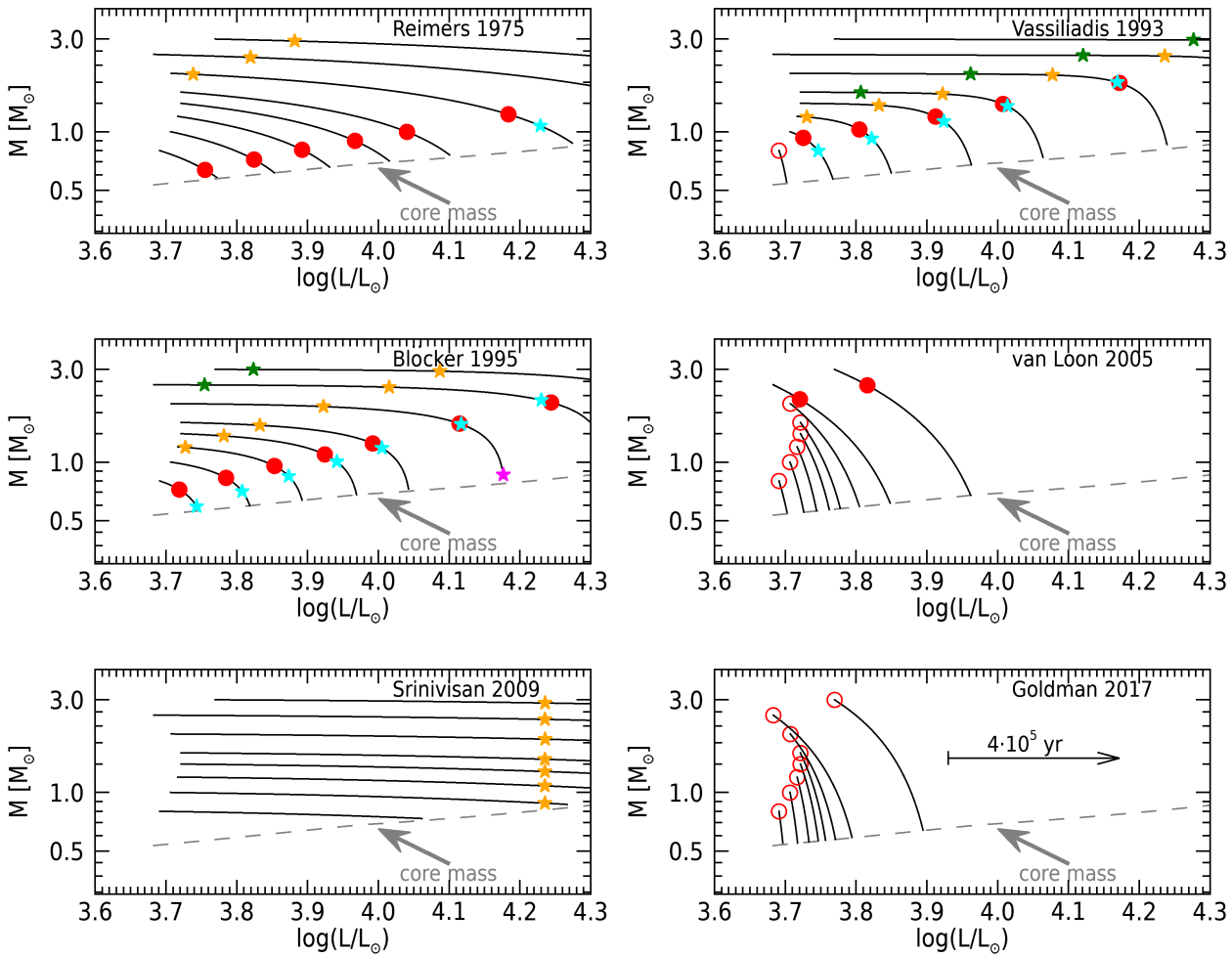

Figure 6

Evolution in mass and luminosity for stars with a solar composition for different mass-loss rate prescriptions. The different tracks show the stellar evolution for AGB stars with a mass at the first thermal pulse of $(0.8,1.0,1.2,1.4,1.6,2.0,2.5,3) \mathrm{M}_{\odot}$. In each panel, a different mass-loss rate prescription has been used, as indicated in the top right corner. The core-mass-luminosity relation is displayed by the dashed gray line. The filled red circles indicate the locus where $\mathrm{d} \log \mathrm{M}_{\star} / \mathrm{d} t=\mathrm{d} \log \mathrm{L}_{\star} / \mathrm{d} t$; an open red circle implies $\mathrm{d} \log \mathrm{M}_{\star} / \mathrm{d} t$ is larger than $\mathrm{d} \log \mathrm{L}_{\star} / \mathrm{d} t$ at the start of the calculation. The coloured stars specify the locus where $\dot{M}$ is equal to $10^{-8}$ (green), $10^{-7}$ (orange), $10^{-6}$ (cyan), $10^{-5}$ (magenta) $\mathrm{M}_{\odot} \mathrm{yr}^{-1}$. The rate of change of the star's abscissa in this plot is $\mathrm{d} \log \mathrm{L}_{\star} / \mathrm{d} t=0.605 \mathrm{Myr}^{-1}$, which is indicated by the black arrow in the lower right panel.

let Willson (2000) identify a strong selection effect concerning stars for which the mass-loss rate is measurable. Stars not yet near the cliff will have low mass-loss rates that are difficult to detect or to measure, while stars beyond the cliff will be short-lived causing a scarcity in the detection rates. This implies a selection bias towards stars near the cliff. Thus, the empirical mass-loss laws 'tell us the parameters of the stars that are losing mass, and not the dependence of the mass-loss rates on the parameters of any individual star' (Willson 2000). Although the 1-dex width in mass-loss rate around the 'cliff' is not necessarily that narrow - all depend on the particular mass-loss rate behaviour over time - Willsons conclusion remains valid: empirical mass-loss rate formulae 'tell us which stars are losing mass rather than how stars are losing mass over time'.

This conclusion by Willson brings us back to the earlier discussion of the benefits (but 
also pitfalls) of the reductionist approach. At the risk of oversimplification, this approach is linked to that termed 'hierarchical reduction'; the idea that phenomena at one hierarchical level can be explained by using concepts from a lower hierarchical level. Thus we conventionally express astrophysical phenomena in terms of chemical principles, and chemical phenomena in physical terms, and physical phenomena in a mathematical language; or as the Nobel Prize physicist Steven Weinberg succinctly expressed 'explanatory arrows always point downward' (Weinberg 1994). Empirical mass-loss rate prescriptions do not follow the arrow downward, but are holistic expressions of an emergent property, and hence take a 'top-down' approach in their attempt to unravel stellar evolution. Indeed, quite confusingly, 'top-down' does not imply 'point downward' in Weinberg's words. The risk of an 'emergent property' is that it raises the expectation that the behaviour is understood, but that is not necessarily true. In this particular case, I would not recommend using empirical massloss rate relations in stellar evolution models, since the seemingly logical relation might be causally wrong. In contrast the forward theoretical approach is 'bottom-up' (and points downward), and there are a number of factors that argue persuasively that the bottom-up approach will change the landscape of mass-loss rates considerably in the next few years. These 'winds of change' come from different cardinal points, each of them inherently linked to the 3D reality of a stellar wind. They are steered by recent progress in quantum chemistry and astrophysical observations, and can now build up momentum thanks to the latest developments in supercomputing capabilities.

I do not want to leave this section with the reader having the feeling that these empirical laws are deceptive or useless. On the contrary! If systematic biases in the retrieval approach can be avoided, the observations tell us the real rates of mass loss. Only after detailed theoretical descriptions are found that reproduce the retrieved rates and relations, may the models be used to extrapolate to populations not presently available for study, such as low-metallicity populations in the early Universe. For only then, we will have greater faith in our predictions of the maximum luminosity achieved by AGB stars; the mass spectrum of planetary nebulae and white dwarfs; the frequency of type I and type II supernovae, and possibly the masses of their progenitors; and the fate of stellar and planetary companions residing close to the mass-losing red giant primary star. But even if theoretical and empirical mass-loss rate relations agree, we must be extremely vigilant against any confirmation bias in our theoretical efforts.

\subsection{Informative measures challenging the 1D world}

Our earlier discussion has identified the explicit dependence of the mass-loss rate on fundamental parameters as the nut that needs to be cracked. From the discussion of Figure $\mathbf{5}$ it is clear that the theoretical and empirical mass-loss rate prescriptions appear to be similar, but they are in fact intrinsically different. There are challenges ahead of us for there to be a simple gateway between both types of relations, but recent progress in observations and quantum chemistry leads us to believe that the gap between them is not cavernous, and a bridge is gradually but assuredly coming into view. The central pillar of that bridge is the incorporation of a $3 \mathrm{D}$ view in all our measures of mass loss, either in the forward or the retrieval approach. As a first step, we need to question whether the retrieved mass-loss rates and relations are reliable. Here recent progress in observational techniques indicate that there is an elephant in the room - well actually two elephants - a smaller and a bigger one, which are caught in the act owing to the incredible capacities of novel high spatial
Type I or Type II supernova: a supernova is classified as type II if the spectrum displays the hydrogen Balmer lines, otherwise it is Type I

Type la supernova: when a white dwarf is triggered into a runaway nuclear fusion, caused by the accretion of matter from a binary companion or a stellar merger

Type Ib/c and Type II supernova: caused by the gravitational collapse of the core of a massive star, resulting in a black hole or neutron star 
resolution observing capabilities. On the one hand, there are flow instabilities induced by convection that result in the formation of granulation cells on the surface of the giant stars, and of small-scale density structures in the stellar wind, with sizes of between $\sim 1-50$ au (see Figure 7). On the other hand - and encompassing much larger geometrical scales - there is mounting (indirect) evidence that most evolved cool giant stars with measurable mass-loss rates are surrounded by at least one stellar or planetary companion. The companion will perturb the structure of the stellar wind (see Figure 7), and will under some circumstances induce an increase in mass-loss rate. As I will discuss in Section 4.3 . neglecting the $3 \mathrm{D}$ structural complexities in retrieval approaches can lead to mass-loss rates that are incorrect by an order-of-magnitude, which — as we have seen in Figure 6 — has a huge impact on the outcome of stellar evolution models.

This discussion on the emergence of 3D clumps and the impact of a (hidden) companion provides insight into the deterministic perspective of cool ageing stars and their contribution to the chemical enrichment of the Universe. However, there is an additional approach to the how question that could potentially provide useful information for the why question as well. By probing what we believe are the early beginnings of a stellar wind, we might be able to grasp the essence of mass loss. To do that, we have to strip the outer layers of complexity, the large 3D structures with scales greater than $1 \mathrm{au}$, to uncover what lies beneath. We need to reverse the time axis and dive deeply into the smallest $3 \mathrm{D}$ scales that matter. Doing so, we reach the nanometer-sized domain where dust nucleation is happening (see Figure 7). Arriving there, we will discover that, here as well, the bottom-up approach has recently been very instructive in providing new insights in the intricacies of this phase transition, but it requires us to embrace the complexities of the $3 \mathrm{D}$ structures of molecules, large gas-phase clusters and dust grains. Once we arrive at that scale, we may begin to understand why cool ageing stars contribute to the galactic chemical enrichment and what are the driving forces for the complex physicochemical processes governing the Universe. However, it also will become clear that untangling the tangled web of Schrödinger's equations - describing the transition from molecules to dust - still lies in the distant future.

\section{The emergence of 3D clumps}

The first unambiguous observation of structure on the stellar surface of a star other than the Sun was reported in 1990, when Buscher et al. presented the detection of a bright surface feature on the surface of the red supergiant Betelgeuse. Betelgeuse has one of the largest apparent angular sizes in the night sky (with a measured angular diameter of $\sim 44.2$ mas in the infrared, Dyck et al. 1992), and is an ideal candidate for spatially resolving its surface, not only in the optical, but also in the UV and sub-millimeter wavelength domain (Gilliland \& Dupree 1996, O'Gorman et al. 2017). Gilliland \& Dupree (1996) interpreted the observed feature as resulting from magnetic activity, atmospheric convection, or global pulsations and shock structures that heat the chromosphere. Recently, another profound realisation in observational astronomy occurred through the first image of large granulation cells on the surface of a (much smaller) AGB star, $\pi^{1}$ Gru; our Sun might show some resemblance to $\pi^{1}$ Gru once it becomes a red giant star in about 7.7 billion years. While the surface of the Sun is covered with about two million convective cells whose typical size is around $2000 \mathrm{~km}$ across, $\pi^{1}$ Gru was shown to have only a few granules with a characteristic horizontal size of about $1.2 \times 10^{8} \mathrm{~km}$, or $\sim 27 \%$ of the stellar diameter (Paladini et al. 2018). These observations are consistent with the historical prediction by Schwarzschild 


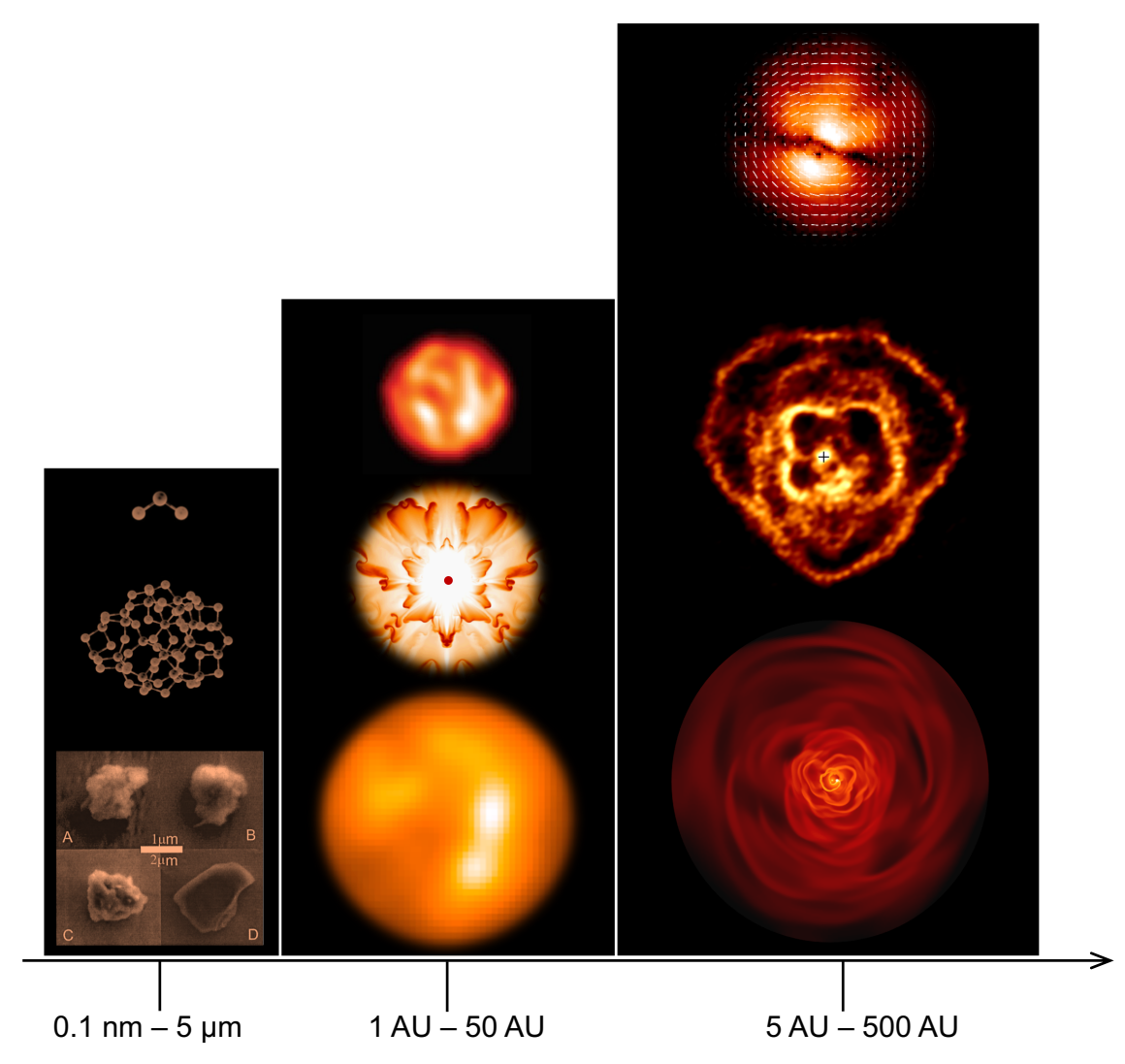

Figure 7

3D structures of relevance for stellar wind research. Compilation based on observations, theoretical calculations, modelling, and laboratory experiments. First column: the 3D life of molecules and dust grains: $T o p-\mathrm{TiO}_{2}$ proposed as candidate for the first dust condensate in O-rich winds (Jeong et al. 2003, Lamiel-Garcia et al. 2017); Middle - global minima candidate for $\left(\mathrm{TiO}_{2}\right)_{n}$ with $n=24$ obtained within a bottom-up approach by using a global optimisation algorithm searching the complex multidimensional potential energy surface (Lamiel-Garcia et al. 2017); Bottom presolar corundum grain with AGB origin, image obtained using a scanning electron microscope by Choi et al. (1998). Second column: the emergence of 3D clumps. Top - due to convection in the stellar atmosphere large granulation cells form on the surface of the O-rich AGB star $\pi^{1}$ Gru and are now seen thanks to the PIONIER instrument mounted at the Very Large Telescope Interferometer (VLTI; Paladini et al. 2018); Middle - flow instabilities arising in a 2D model for a dust-driven wind of a carbon star (Woitke 2006a), the red dot represents the AGB star; Bottom $2.166 \mu \mathrm{m}$ image of the carbon star CW Leo obtained with NACO, mounted at the Very Large Telescope (VLT), showing the presence of small-scale structures (clumps) embedded in the stellar wind (Menut et al. 2007). Third column: the 3D lasting impact of a companion. Top - detection of an equatorial dust lane in the wind of the carbon star CW Leo using scattered light data obtained by ExPo mounted on the William Herschel Telescope (Jeffers et al. 2014); Middle - a rose-like spiral structure detected in the stellar wind of the O-rich AGB star R Hya using ALMA (Decin et al. 2020); Bottom - 3D hydrodynamical simulation for a binary system containing a mass-losing AGB star (El Mellah et al. 2020). 
(1975) that the surface of cool giants might be covered by a relatively small number of giant convection shells. Schwarzschild came to his hypothetical conclusion on the basis of the working hypothesis that the pressure or density scale-heights determine the size of the convective elements. Moreover he addressed the question of whether mass ejection could be triggered by photospheric convection. He argued in favour of that suggestion on the basis of observations which provided substantial evidence for non-spherical circumstellar dust clouds in the neighbourhood of red giant and supergiant stars, in which the polarized light signal shows variability on the same time scale (of $\sim 200$ days) as the irregular brightness variations caused by the giant convective cells. The cooler regions of the large-scale surface convective elements might enhance the production of dust grains resulting in an uneven distribution of dust and hence of the polarization signal. With this section revolving around the deterministic question and hence aspects of mass loss, we seek to answer whether, in principle, Schwarzschild's argument is settled, and if and how these non-spherical circumstellar dust clouds can be used to trace the mass-loss mechanism.

\subsection{Weather map from cool ageing stars: dry with variable cloud cover}

The conjecture of Schwarzschild that there is a potential causal link between the dynamical, inhomogeneous stellar atmosphere driven by convective flows and the formation of clumpy clouds in the CSE can be checked against observational evidence. In the early seventies there was only indirect evidence of non-spherical dust clouds in the close vicinity of cool ageing stars (Shawl 1972, Schwarzschild 1975), but the new revolution in observational techniques allowed for high-spatial resolution (reconstructed) images and provided direct observational evidence that the CSEs harbour small and large-scale inhomogeneities (for a discussion of large correlated density structures, see Section 4). The CSE of the carbon-rich AGB star CW Leo (at a distance of $\sim 150 \mathrm{pc}$ ) has long been known to be quite complex and continually evolving (see Figure 7). As the IR brightest AGB star experiencing high mass loss, it has been exhaustively studied by many observational techniques. Fine structure on sub-arcsec scale was detected within $\sim 30$ stellar radii from the central star (Weigelt et al. 1998, Haniff \& Buscher 1998). The fragmentation of the shell in distinct clumps was suggested to be caused by inhomogeneous mass loss potentially induced by large-scale surface convection cells. However, the stellar surface and inner CSE of this star are obscured by the optically thick envelope of carbon dust, hindering the identification of the observed clumpy features with processes occurring at the stellar surface, in the shock-dominated inner CSE, and in the dust-formation region. Even for carbon stars with a more modest mass loss, IR images can be difficult to interpret, as was shown recently by the example of R Scl (Wittkowski et al. 2017).

The envelopes of O-rich giants tend to be more transparent in the visual and infrared wavelength regime than C-rich giants (see also Section 5), thereby providing better access to the surface and innermost CSE layers and hence allowing for a more detailed evaluation of Schwarzschild's conjecture. Observations of scattered light of nearby O-rich AGB and RSG stars establish there are dust clouds as close as $\sim 0.5 R_{\star}$ to the star, whose size is $\lesssim 20 R_{\star}$ and which change in morphology on time scales of weeks to months (Khouri et al. 2016, Ohnaka et al. 2017a, Adam \& Ohnaka 2019, Kamiński 2019, Cannon et al. 2020). The dynamical time scale implied by changes in the circumstellar morphology are in good agreement with the characteristic time scales for convection; and the observed spatial scales (both the size of the clouds and the distance from the stellar surface) compare well with recent $3 \mathrm{D}$ radiation 
hydrodynamic models simulating the outer convective envelope and dust-forming region (Höfner \& Freytag 2019, see also Section 3.2 for some side notes on this claim). Thanks to these results, we have increased confidence that the Schwarzschild's conjecture actually might reflect reality. However, the recent two-dimensional mapping of the velocity field over the surface and inner CSE of the nearby red supergiant Antares throws a spanner in the works, because the maps reveal vigorous upwelling and downdrafting motions of several huge clumps of gas with velocities ranging from about -20 to $+20 \mathrm{~km} \mathrm{~s}^{-1}$ in the inner CSE up to $\sim 1.7 \mathrm{R}_{\star}$ (Ohnaka et al. 2017b). Convection alone cannot explain the observed turbulent motions and atmospheric extension, suggesting that a process which has not yet been identified is operating in the extended atmosphere (Arroyo-Torres et al. 2015, Ohnaka et al. 2017b). Admittedly, this result does not rule out Schwarzschild's conjecture, but it reveals that the apparent analogy and the correlation between the surface granulation pattern and the gas and clumps in the extended atmosphere and inner CSE does not in itself signify a causal relation and conceivably other processes might be important.

\subsection{How to model a turbulent life}

Whether we consider either a small Sun or a giant star, modelling the turbulent dynamical process of convection and its interaction with other physical, chemical and radiative processes, is a major challenge. This is not only due to the complexity of the problem, but is also due to the huge CPU demand dictated by the numerical resolution required for a proper sampling of the time-dependent small- and large-scale fluctuations in density, temperature, velocity, and brightness. Detailed radiation hydrodynamics (RHD) simulations can help to understand qualitatively these processes and to model quantitatively the dynamical layers in and around these stars (Freytag et al. 2019). Modelling convection and surface granulation in sun-like stars is only possible using local 3D RHD simulations owing to the huge disparity in spatial and time scales: for a global simulation of a sun-like star one would need a spatial resolution of at least a fraction of the photospheric pressure scale height of $\sim 150 \mathrm{~km}$ to cover the Sun (diameter of about $1400000 \mathrm{~km}$ ), and a time resolution of about a second for at least several rotation periods of about a month or, even better, several magnetic cycles, each spanning 22 years (Freytag et al. 2019). In contrast, global 3D RHD models covering the entire convective surface of cool giants are within the realm of possibility, because of Schwarzschild's prediction that only a few giant convection cells cover their surfaces. The first global 3D RHD simulations for a red supergiant were presented in 2002 (Freytag et al. 2002), the domain of the smaller AGB stars was reached in 2017 (Freytag et al. 2017). These models encompass part of the atmosphere with the outer boundary situated at $\sim 2 \mathrm{R}_{\star}$. The model dynamics are governed by the interaction of long-lasting giant convection cells, short-lived surface granules, and radial fundamentalmode pulsations (see also Section 2.2.3. The models did not yet include dust formation and therefore no wind driving. Recently, a global model for an oxygen-rich AGB star was presented that incorporates both the outer convective atmosphere and the dust-forming region up to $\sim 2.8 \mathrm{R}_{\star}$ (Höfner \& Freytag 2019). The current models do not yet describe wind acceleration and the kinetic treatment of grain growth does not account for nucleation, but assumes the presence of seed particles. I will expand on the challenge of dust nucleation shortly (Section 5), but for the time being it is sufficient to realize that we are still speculating as to the specific steps of seed formation. In the models grain growth is hypothesized to take place under the most optimum circumstances (sticking coefficient assumed to be 
Supersaturation: a gas-solid or gas-liquid chemical system which is in a non-equilibrium state such that there are too many gaseous molecules for the present temperaturepressure conditions one; see also Section 5.3.1.

Although the 3D RHD models including dust growth still have some limitations, already they offer great insight into the essence of mass loss at least for stars on the upper AGB and in the RSG phase where stars have the lowest surface gravities, and hence largest pressure scale heights and largest granules relative to the stellar radius. The large-scale convective flows and pulsations generate atmospheric shock waves: the shorter-wavelength disturbances cause a complex small-scale network of shocks in the innermost layers, while the fundamental-mode pulsation causes a more or less spherical shock front that is able to travel further away from the stellar surface. In the dense wake of the shock, gas is temporarily lifted to distances where dust formation may occur (Freytag et al. 2017). Remarkably and importantly, the temperature shows a rather smooth, almost spherical pattern, in contrast to the gas densities, which are strongly affected by the local 3D dynamics and show a pronounced 3D clumpy morphology (Höfner \& Freytag 2019). Only when the temperature falls below a critical value does the gas become supersaturated. Under these circumstances the molecules are more prone to leave the gas than to rejoin it, so they become deposited on the surface of the solid particles and grain growth is triggered. Since the temperature acts as a threshold for the onset of grain growth, while the gas densities determine the grain growth efficiency, the models show an almost ring-like dust number density distribution conditioned by the local temperature. For a $2 \mathrm{D}$ slice through the center of the grid, the dust layers appear narrow in the radial direction due to the rapidly decreasing densities with increasing distance. Even then, smaller patches of dust clouds appear where both the density and radius of the grains vary in response to the local gas density and velocity. This behaviour is not only characteristic for O-rich environments, but $2 \mathrm{D}$ carbon-rich wind models also show a similar pattern (Woitke 2006a see Figure 7).

Given the fact that the models of Freytag et al. (2002, 2017) and Höfner \& Freytag (2019) do not include the radiation pressure on dust grains, they do not directly address the speculation by Schwarzschild that mass ejection could be triggered by photospheric convection. However, given the results just described I argue here that clumps generated by convective motions are not the cause or the essence of mass loss, rather they are the consequence of the local 3D atmospheric conditions. This argument is based on i) examination of the isotherms, which are set by non-local radiative processes, being nearly perfect spheres and ii) the consideration that not only the onset of the dust growth, but also, even more elementally, dust nucleation is dictated by the local temperature (see Fig. 8 in Woitke 2006a and also Section 5.3.2. Convection-induced 3D cloudy structures are of second order for wind driving in the sense that grain growth can be locally of higher or lower efficiency due to a change in density, but as long as the gas is not supersaturated no force can be generated which overcomes the stellar gravitational attraction. This argument rests on the common idea of a dust-driven outflow, which is more appropriate for AGB than RSG winds. For RSG stars with their very low surface gravities the situation might be the reverse. A recent study shows that inferred atmospheric turbulent velocities yield turbulent pressure high enough to initiate mass loss even in the absence of circumstellar dust (Kee et al. 2020). In this regard, and for these fluffy RSG stars, Schwarzschild's conjecture still holds — but is not yet proven - and photospheric convection seems to be a viable mechanism to trigger mass ejection. 


\subsection{Impact on derived properties}

Clumps show up not only in the inner CSE, but they are also present at much larger distances from the star - i.e., up to the bow shock, where the stellar wind collides with the ISM (Cox et al. 2012, Decin et al. 2016, 2018b, Montargès et al. 2019, Kamiński 2019). At present, it is still not understood which mechanism prevents clumps that are formed in the inner CSE, from dissipating during their travel through the huge CSE. Potentially, clumps can cool very efficiently inducing a reduction of the internal pressure. But even without understanding the process, we are in principle able to quantify the impact of $3 \mathrm{D}$ clumps on mass-loss rate retrieval efforts by carefully measuring the amount of mass in the clumps and the surrounding smooth envelope. However, the need for highly performant $3 \mathrm{D}$ radiative transfer analysis often steers scientists towards simplistic analytic estimates. A small mistake in the optical depth, however, might have a substantial impact on the mass estimates because the optical depth enters in the exponent of the intensity estimate $\left(I_{\nu} \propto S_{\nu} \exp \left(-\tau_{\nu}\right)\right)$. This leaves us with only a few examples, all RSG stars, to guide us in this exercise. These analyses indicate that clumps contribute from a few up to $\sim 25 \%$ of the total mass loss (Ohnaka 2014, Montargès et al. 2019, Cannon et al. 2020), the only potential exception is the extreme RSG VY CMa for which the derived dust mass in the clumps (of $0.47 \mathrm{M}_{\odot}$ ) seems unrealistically high to be compatible with a current stellar mass of $17 \mathrm{M}_{\odot}$ (Kamiński 2019). With the exception of VY CMa, this suggests that the mass loss in ejected clumps contributes non-negligibly to the total mass loss, but also that the clumps do not represent the main mass-loss mechanism. This conclusion reinforces the final statement in the preceding section: convective-induced turbulent pressure might invoke mass ejection for RSG stars, but in all its generalities that mechanism only depends on the turbulent velocities and has no significant explicit dependence on any 3D clump characteristic.

Given this (tentative) outcome and the substantial struggle for deriving reliable massloss rate relations (as described in Section 2.2.4), it seems fair to conclude that the story of $3 \mathrm{D}$ clumps only bears limited relevance for both the deterministic and conceptual question. However, this is too short-sighted for several reasons. First, forward theoretical models predict a considerably different molecular abundance pattern whether clumps are included or excluded (Agúndez et al. 2010, Van de Sande et al. 2018b), where notable examples include the formation of water $\left(\mathrm{H}_{2} \mathrm{O}\right)$ molecules in a carbon-rich wind and hydrogen cyanide $(\mathrm{HCN})$ in O-rich envelopes at sufficiently high abundances to be detected with very sensitive telescopes. These predictions are not only consistent with contemporary observations (e.g., Lombaert et al. 2016, Van de Sande et al.2018a), but they also convey the message that our estimate of the chemical enrichment of the ISM by cool ageing stars is at best preliminary.

Second, stars do not stand still. Instead stars travel through the ISM, where a striking testimony is the appearance of bow shocks that are so beautifully imaged with the Herschel Space Observatory (Cox et al. 2012). The difference in velocity and density between both media invoke the growth of Rayleigh-Taylor and Kelvin-Helmholtz instabilities, and kinetic temperatures are estimated to reach up to $10000 \mathrm{~K}$, depending on the shock velocity $v_{S}$ (van Marle et al. 2011, Decin et al. 2012). This might foster the dramatic idea that the collision with the ISM can destroy any relic of the chemical processes occurring in stellar winds, because molecules can break up (dissociate) and grains can be destroyed. However, this is only true for the most violent collisions and even then the larger grains are shown to survive (van Marle et al. 2011), leaving us with a grain size distribution modified toward larger grains. For low-velocity collisions $\left(v_{S} \leq 5 \mathrm{~km} \mathrm{~s}^{-1}\right)$, most of the kinetic energy dissipates via magnetic compression and through the rotational emission of molecules such as CO 
Post-AGB star: when the envelope mass of the AGB star constitutes less than $\sim 1 \%$ of the stellar mass, the star becomes a post-AGB star; a phase which only takes a few thousand years before transiting to the $\mathrm{PN}$ phase and $\mathrm{H}_{2} \mathrm{O}$ or the fine structure lines of $\mathrm{C}^{+}$and $\mathrm{O}$ (Godard et al. 2019). As the velocity increases, $\mathrm{H}_{2}$ becomes the dominant coolant over a wide range of shock velocities and gas densities (Lesaffre et al. 2013, Flower \& Pineau des Forêts 2015). The general outcome is a higher fraction of ionized atoms, excited molecules, and sputtered grains. Returning to the persistent clumps and their importance for the deterministic aspect, if material is embedded within higher density clumps it will be less affected by the collisions which occur in the bow shock, by the harsh interstellar UV field, and by the possible entwinement of radiative and mechanical energies. In other words, clumps not only help us diagnose the genuine wind chemistry, they are also a key ingredient for quantifying the galactic chemical evolution and are, as such, of fundamental importance for the deterministic question.

\section{The 3D lasting impact of a partner}

So while the smaller elephant (a.k.a. the 3D clumps) seems part of the furniture with limited repercussion on mass-loss rate estimates - but with a profound effect on chemical processes - there is still that other, bigger elephant, which came into view owing to the detection of large correlated density structures in stellar winds, and which exposes the scientific quandary that modern astrophysics has been contending with in recent years. It has been more often than not that unexplained phenomena in observational astrophysics were either 'justified' or conversely 'neglected' by using the phrasal idiom '. . . binarity which is not within the scope of this paper (or conference talk)'; and magnetic fields suffer the same fate. Our Sun with its eight planets has no (known) stellar companion. So akin to social psychology, where it is well known that humans tend to hire job candidates on the basis of similarities to themselves (the 'similarity attraction bias'), a general thesis that formed the basis for stellar evolution models took shape, namely that solar-like stars live their lives alone (and the planets are inconsequential for the late stages of evolution). In retrospect that thesis now appears to be problematic, especially if we are pondering the fate of solar-like stars experiencing substantial mass loss on the AGB. I will venture the idea that a planetary or stellar companion impacts the wind morphology of almost all AGB and RSG stars with a detectable mass-loss rate $\left(\dot{M} \gtrsim 10^{-7} \mathrm{M}_{\odot} \mathrm{yr}^{-1}\right)$. Moreover, in a fraction of stars the companion induces a change in expansion velocity and mass-loss rate. This implies that most empirically derived mass-loss rates are obtained from samples containing a large fraction of stars that experience binary interaction with a (sub-)stellar companion. Therefore, our knowledge of the mass-loss rate is biased by the impact that companions can have on the strength of the mass loss and on the observed diagnostics from which mass-loss rate values are retrieved. It goes without saying that this viewpoint touches directly on the deterministic question, and - unlike the small elephant - this time we will not be bogged down in the minutiae of detail; a partner changes your life once and for all.

\subsection{The key to finding the invisible partner}

Binary companions orbiting mass-losing AGB stars somehow managed to escape the scientific picture. Every so often the presence of companion was invoked to explain the metamorphosis from an overall spherical AGB wind to the aspherical morphologies seen in their descendants, the post-AGB stars and the planetary nebulae (PNe). Indeed, already the first photographic atlas of $\mathrm{PNe}$, published in 1918, showed an astonishingly wide range of PNe morphologies and led Curtis to question whether 'it is possible to postulate any general form or forms, which shall be mechanically plausible, and to which the planetaries, 
or a considerable proportion of the planetaries, will more or less closely conform?'. Since then, the quest for the primary mechanical cause shaping PNe has been open. Whereas $\sim 80 \%$ of AGB stars have a wind with overall spherical symmetry (Neri et al. 1998), less than $20 \%$ of PNe possesses a circular symmetry (Parker et al. 2006. Sahai et al. 2011). Naming only a few prominent examples imaged by, for example, the Hubble Space Telescope (HST): the inner structure of the Eskimo Nebula, a bipolar double-shell PN, shows a complex 'roselike' structure (O'Dell et al. 2002), while the Helix Nebula is a bipolar PN with an 'eye'-like morphology (O'Dell et al. 2004, Su et al. 2007). Biconical shapes are seen in various postAGB stars and PNe, including the post-AGB star IRAS 17150-3224 which also has a highly equatorially enhanced shell (Ueta et al. 2007) and the Owl Nebula (NGC 3587), a PN which has a barrel-like structure in its inner shell caused by bipolar cavities (Guerrero et al. 2003). The Red Rectangle is an O-rich post-AGB binary system which has a Keplerian (rotating) disk and an outflow, the latter mainly being formed of gas leaving the disk (Bujarrabal et al. 2016); in its outer halo regularly spaced arcs embedded in a bipolar outflow are detected (Cohen et al. 2004).

For a long time, the development of these nonspherical structures during the postAGB and PNe phase has been a matter of debate. Several contending theories attempted to explain this morphological metamorphosis, including rapid stellar rotation and strong magnetic fields in single-star models (García-Segura et al. 1999), and binary models with a particular focus on short-period binary systems formed after a common-envelope phase (orbital period $P_{\text {orb }} \lesssim 10$ days; Soker 1998, Miszalski et al. 2009). Even for the first two mechanisms, the presence of a binary companion is sometimes called upon to sustain the mechanism (Soker \& Harpaz 1992). In principle, the material ejected during the AGB phases does not have enough angular momentum to form Keplerian disks, which should only appear around binary stellar systems, as these systems have the necessary angular momentum stored in their orbital movement. Axial structures in the form of collimated fast winds have then been proposed to be associated with such rotating disks, from which material would fall onto the star or a companion during early post-AGB phases, powering very fast and collimated stellar jets (Bujarrabal et al. 2001, Soker 2001, Balick \& Frank 2002). Most of these proposed PN shaping processes act on the AGB star itself and each of them is thought to operate over a short time, either during the final few hundred years of the AGB phase or during the early post-AGB phase. The short lifetime of the post-AGB and $\mathrm{PN}$ phases, the strong observational bias toward detecting short-period binary postAGB stars and PNe, and the high mass-loss rates at the end of the AGB phase leading to an obscuration of the inner workings made the identification of the shaping mechanism and its time of occurrence observationally challenging.

Aiming to understand some of the observable characteristics of axisymmetric or bipolar post-AGB stars and PNe, Mastrodemos \& Morris (1999) were the first to perform pioneering hydrodynamical simulations of dusty red giant winds in detached binary systems $\left(P_{\text {orb }} \gtrsim\right.$ $1 \mathrm{yr})$, in which the effects of the companion are manifested on the wind of the red giant rather than the star itself. (See the sidebar titled Binary interaction.) In these simulations, the primary star was a mass-losing red giant star and the companion's mass ranged between $0.25-2 \mathrm{M}_{\odot}$ with orbital separation between $3-24 \mathrm{R}_{\star}$. They derived a range of envelope geometries encompassing bipolar, elliptical and quasi-spherical geometries characterized by a continuously decreasing density contrast between the equatorial plane and the poles. The last category manifested a novel type of hydrodynamic wind solution in the form of a spiral shock caused by the reflex motion of the mass-losing AGB star. Depending on the system's 
parameters and the inclination angle of the system, the two-dimensional projection of these spirals in the plane of the sky appears as an Archimedes spiral or as a series of rings in the wind. The year 1999 was also when the first-ever multiple, incomplete, concentric shells were detected in the envelope of the carbon star CW Leo $\left(\dot{M} \sim 1.5 \times 10^{-5} \mathrm{M}_{\odot} \mathrm{yr}^{-1}\right)$ using the HST (Mauron \& Huggins 1999). Probably unaware of the results by Mastrodemos \& Morris (1999), Mauron \& Huggins attributed the shells to mass-loss modulations with a time scale of $\sim 200-800 \mathrm{yr}$, caused by an undefined episodic process intrinsic to the star. The impact of a binary companion was tentatively suggested, but was deemed insufficient to explain the structure of the shells farther out. Searching for the cause of the episodic process, Simis et al. (2001) suggested that an intricate non-linear interplay between gasgrain drift, grain nucleation, radiation pressure, and envelope hydrodynamics can result in gas and dust density shells that occur at irregular intervals of a few hundred years for a single-star model. However, the predicted shell density variations were much larger than the observed ones (Decin et al. 2011).

It took an additional seven years before the same authors, Mauron \& Huggins, reported in 2006 the detection of the first conspicuous Archimedes spiral pattern in the wind of a carbon-rich AGB star (AFGL 3068, $\dot{M}=4.2 \times 10^{-5} \mathrm{M}_{\odot} \mathrm{yr}^{-1}$ ). This discovery was, and still is, highly significant and led to the unequivocal conclusion that binary interaction shapes the stellar wind during the AGB phase, and as such plays a role in carving out planetary nebulae. However, despite presenting this and analogous results (see below) to the scientific community, there still remains a fraction of skeptical astronomers because the companion remains invisible to current instrumentation. As a renowned German astrophysicist told me after my lecture on 7 February 2020, 'You know, Leen, it all looks so fantastic, the observations are so fascinating, the current state-of-the-art models seem to do a pretty good job for interpreting the data, but in the end shouldn't we only believe what we actually can see?'. I remember that fraction of a second during which the old saying of Edgar Allan Poe (1845) jumped into my mind 'Believe nothing you hear, and only one half that you see', but finally found myself arguing that much of astrophysics remains in the realm of conjectures, even Einstein s theory of general relativity (1915) or, to bring it closer to my own field of expertise, even the fact that we believe that AGB stars are the ancestors of planetary nebulae. In a few exceptions, such as o Ceti or W Aql, the binary companion is actually seen (Karovska et al. 1993, Ramstedt et al. 2011), but the high luminosity outshines the faint companion for most sources. The Gaia parallax' measurements can potentially give us some light at the end of the tunnel if corrections can be made for the turbulent AGB and RSG atmospheres (Kervella et al. 2019).

The opening of the Atacama Large Millimeter/submillimeter Array (ALMA) in 2012 heralded a leap forward in the understanding of wind morphologies. Observational evidence was steadily accumulating that AGB and RSG winds exhibit large correlated density structures - including arcs, shells, bipolar structures, tori, rotating disks, and spirals embedded in a smooth, radially outflowing wind (see Figure 7 , Maercker et al. 2012 , Ramstedt et al. 2014, Kim et al. 2015, Decin et al. 2015, Kervella et al. 2016, Wong et al. 2016, Guélin et al. 2018, Homan et al. 2018b a, Bujarrabal et al.|2018, Ramstedt et al. 2018, Decin et al. 2019, Randall et al. 2020). At the same time, we saw a revival of hydrodynamical simulations for binary systems in which the primary is a mass-losing AGB star. Depending on the parameters of the system - such as binary separation, mass ratio, eccentricity, rotation and pulsations of the AGB star, mass-loss rate, wind velocity, ... - a wide variety of morphologies is predicted, including: (1) a spiral structure caused by the orbital 


\section{Binary interaction}

While this is not the place to present a detailed physico-chemical description of binary interaction, the key point is easy to state: the gravitational potential of the companion alters the equation of motion and if the companion is UV-active, which is likely if it has lost its envelope, it can impact the chemistry of the surrounding envelope. The concept of Roche-lobe overflow (RLOF) has proven powerful in the description of binary evolution. The critical equipotential surface in the Roche potential, passing through the inner Lagrangian point $L_{1}$, defines two Roche lobes surrounding each star. The volume averaged radius of the Roche lobe can be approximated to an accuracy of better than 1\% following Eggleton's formula (1983):

$$
\frac{R_{L 1}}{a}=\frac{0.49 q^{2 / 3}}{0.6 q^{2 / 3}+\ln \left(1+q^{1 / 3}\right)},
$$

where $q$ is the mass ratio $M_{1} / M_{2}$, and $a$ the orbital separation. Mass-transfer interaction from the primary to the secondary can be classified into four types in increasing order of interaction: Bondi-Hoyle-Lyttleton (BHL) accretion (Hoyle \& Lyttleton 1939, Bondi \& Hoyle 1944), wind-Roche lobe overflow (WRLOF; Podsiadlowski \& Mohamed 2007), Roche lobe overflow (RLOF) when the Roche lobe surface is connected (Paczyński 1971), and common envelope evolution (CEE; Ivanova et al. 2013). It is believed that for wide binary systems $(a \geq 2 \mathrm{au})$ the mass transfer does not occur through RLOF, but via WRLOF in which case wind material from the mass-losing AGB star fills the giant's Roche lobe and is transferred to the companion through a compressed channel which generally does not pass through $L_{1}$.

motion of the mass-losing AGB star around the common center-of-mass, or by the accretion wake of the companion owing to a Bondi-Hoyle-Lyttleton (BHL) flow (see Figure 8, that can be bifurcated for a non-circular orbit; (2) a circumbinary disk; (3) an accretion disk around the secondary; (4) a bipolar outflow, that can display a ripple-like structures if the AGB pulsations are included; (5) an equatorial density enhancement (EDE), with a regular (Keplerian) or complex velocity vector field; (6) and even 'spider' or 'rose-like' structures (see Figure 7, Kim \& Taam 2012, Chen et al. 2017, Liu et al.| 2017, Saladino et al. 2018, Saladino \& Pols 2019, Kim et al. 2019, El Mellah et al. 2020, Chen et al. 2020). In general, smaller values of the orbital separation or wind velocity and larger companion masses induce stronger interaction. These outcomes can be formally expressed in a similar way as Eq. 13. or Eq. 16, although it is obviously seen that the number of independent parameters is far larger. Therefore, the amount of studies in which the model sensitivities can be tested for a large grid of parameters is still very restricted by the huge demand on CPU time and memory required.

The resemblance between the observed and theoretical morphologies supports the claim that binary interaction is a key architect of AGB and RSG wind shaping. However, it also should be acknowledged that the observations are often more complex than any model prediction, owing to simplification in the models and to the fact that other mechanisms, such as magnetic fields, might contribute to an initial wind anisotropy. Generally, deducing the model functions from the behaviour of a complex system is an inverse problem that is difficult to solve, but I contend that this approach will see a significant growth in the coming decade thanks to increasing CPU power and, in particular, the use of artificial intelligence; see for example the paper by de Mijolla et al. (2019). 
Hydrodynamical model
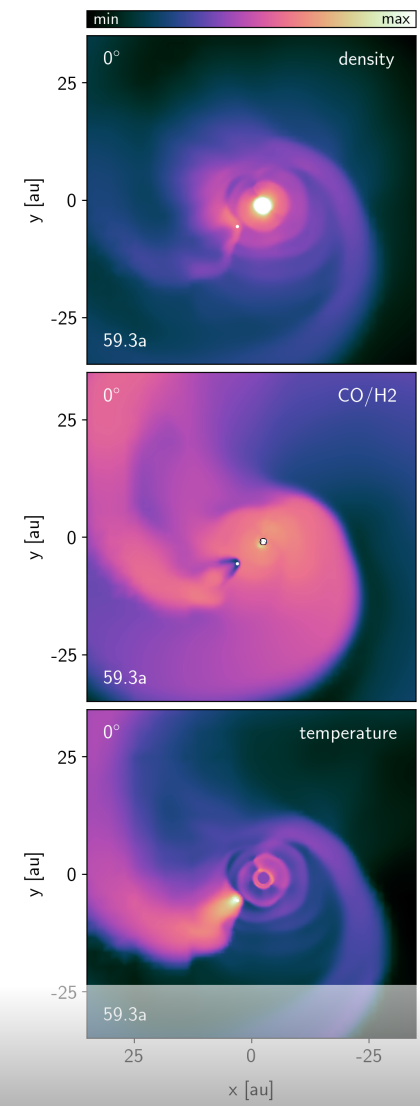

Synthetic observations

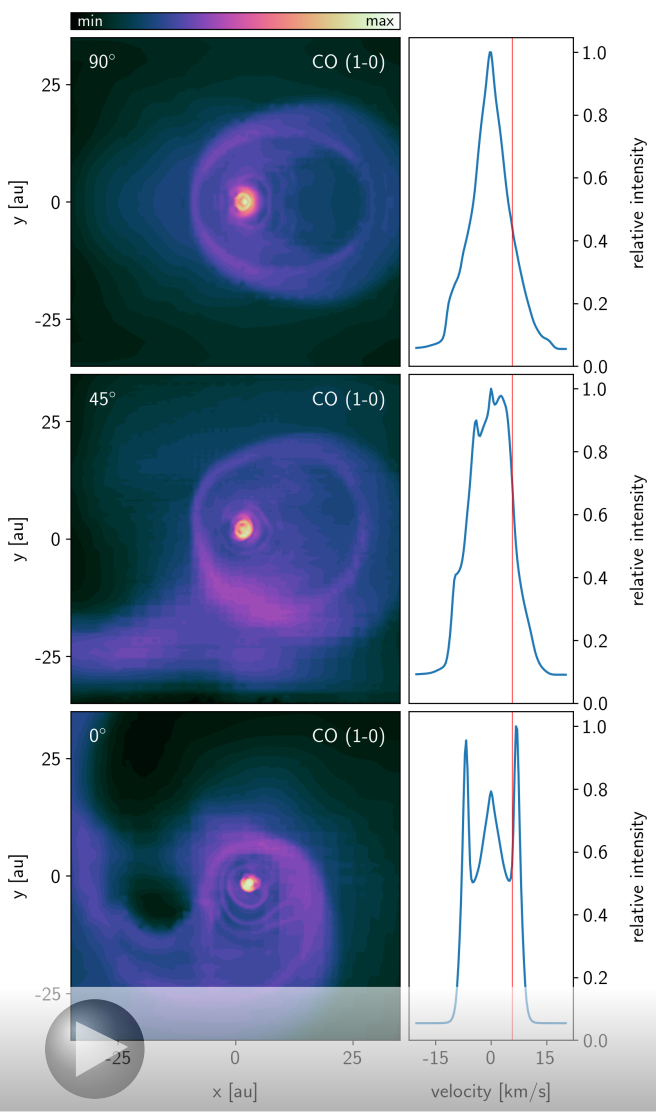

Figure 8

3D hydro-chemical simulation for a binary system containing a mass-losing AGB star. The left panels are a video showing the total density (top panel), $\mathrm{CO} / \mathrm{H}_{2}$ number density (middle panel), and temperature (bottom panel) for a binary system model of which the AGB star has a mass of $1 \mathrm{M}_{\odot}$, effective temperature of $2900 \mathrm{~K}$, a radius of $0.9 \mathrm{au}$, and a pulsation period of 1 year. The companion has a mass of $0.5 \mathrm{M} \odot$ and resides at a circular orbit with separation of $10 \mathrm{au}$. The simulation time runs for $59.3 \mathrm{yr}$. Owing to dust formation occurring in the region where the temperature is lower than $1500 \mathrm{~K}$, a wind is initiated with mass-loss rate of $4.7 \times 10^{-6} \mathrm{M}_{\odot} \mathrm{yr}^{-1}$. The formation of two types of spiral structures can readily be seen, one structure being caused by the gravity wake near the companion, the other one owing to the reflex motion of the AGB star. Both spiral structures merge at larger distances from the AGB star. The small ripples in the close vicinity of the AGB star are relics of the pulsation pattern. The same setup for the AGB star not having a companion yields a mass-loss rate of $7.6 \times 10^{-7} \mathrm{M}_{\odot} \mathrm{yr}^{-1}$ (Bolte et al. in prep.). For a simulation time of $59.3 \mathrm{yr}$, the hydrodynamical quantities are then displayed for viewing angles of the system ranging between $0^{\circ}$ (edge-on view) and $90^{\circ}$ (face-on view). The right panels show the corresponding $\mathrm{CO} \mathrm{v}=0 \mathrm{~J}=1-0$ emission map and line profile at $t=59.3 \mathrm{yr}$ for three different viewing angles (at $90^{\circ}$ - top panel, at $45^{\circ}-$ middle panel, and at $0^{\circ}$ - bottom panel) in the observer's frame calculated using the MAGRITTE 3D radiative transfer solver (De Ceuster et al. $2020 \mathrm{~b}$ a). The video slices through the velocity channel map between -20 to $+20 \mathrm{~km} \mathrm{~s}^{-1}$. Figure courtesy F. De Ceuster and J. Bolte. 


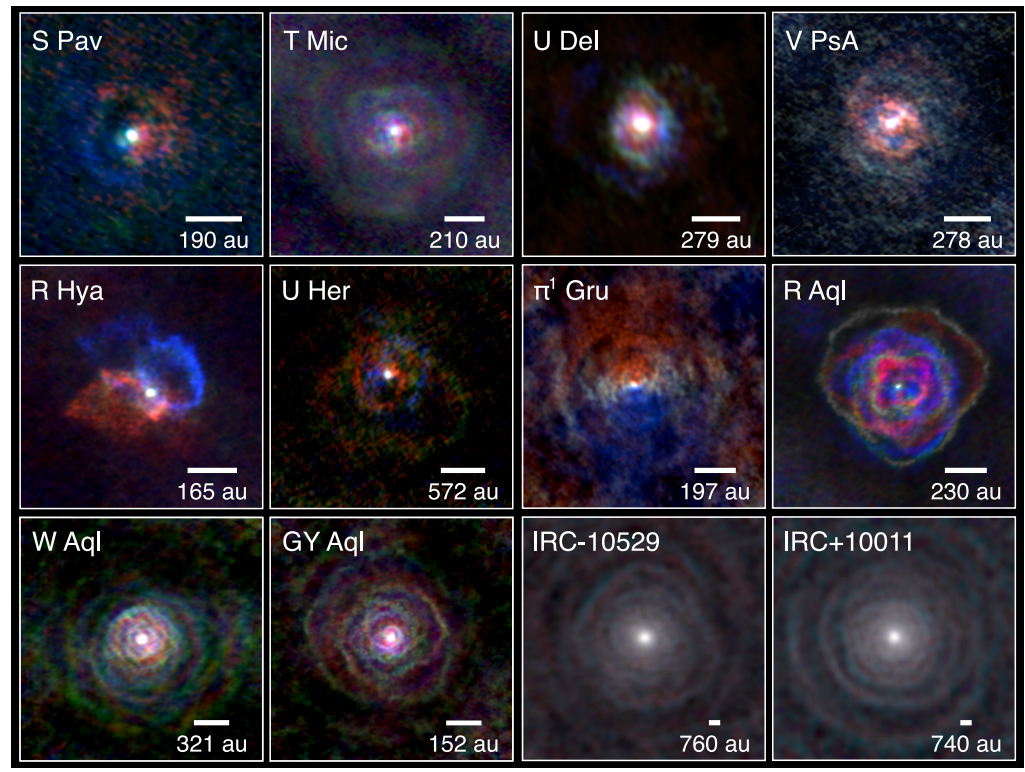

Figure 9

Gallery of AGB winds. Emission maps of 12 AGB stars are shown, derived from the ATOMIUM ${ }^{12} \mathrm{CO} v=0 \mathrm{~J}=2-1$ data. For each star, emission that is redshifted with respect to the local standard of rest velocity is shown in red, blueshifted emission is in blue, and rest velocity is in white. The scale bars have an angular extent of $1^{\prime \prime}$. Sources are ordered by increasing mass-loss rate, from left to right, and from top to bottom. Figure based on Figure 1 of Decin et al. (2020).

Not only will a systematic approach elucidate the intricacies of binary interactions based on theoretical predictions, the same truism holds for observations. In 2018 the ALMA ATOMIUM ${ }^{1}$ Large Programme was granted substantial time on the telescope and a well-selected sample of oxygen-rich AGB and RSG stars was observed in a systematic and unbiased way with the specific aim of understanding the thermodynamical, chemical, and morphological properties of their stellar winds (Decin et al. 2020, Gottlieb et al. 2020). The ATOMium data revealed that all AGB winds observed exhibit distinct aspherical geometries (see Figure 9, which have morphological counterparts in the PNe. This led to the inference that the same physics is key in shaping both AGB winds and PNe. The ATOMIUM data catch the windshaping mechanism in the act and constrain the moment in time when AGB morphologies are being transformed into nonspherical geometries. A strong statistical correlation emerges between the AGB mass-loss rate and the prevailing geometry (Decin et al. 2020): a dynamically complex EDE is often observed for oxygen-rich AGB stars with low mass-loss rates $\left(\dot{M} \lesssim 10^{-7} \mathrm{M}_{\odot} \mathrm{yr}^{-1}\right.$; defined there as 'Class 1 '), a bipolar structure tends to be dominant for stars with medium mass-loss rates ('Class 2'; $10^{-7} \mathrm{M}_{\odot} \mathrm{yr}^{-1} \lesssim \dot{M} \lesssim 10^{-6} \mathrm{M}_{\odot} \mathrm{yr}^{-1}$ ), while the wind of high mass-loss rate stars preferentially exhibit a spiral-like structure ('Class 3'; $\left.\dot{M} \gtrsim 10^{-6} \mathrm{M}_{\odot} \mathrm{yr}^{-1}\right)$. This correlation and the observed characteristics are readily explained by binary interaction. The results allowed Decin et al. (2020) to propose an evolutionary

\footnotetext{
${ }^{1}$ ATOmium: ALMA Tracing the Origins of Molecules In dUst-forming oxygen-rich M-type stars; https://fys.kuleuven.be/ster/research-projects/aerosol/atomium/atomium.
} 
scenario for AGB wind morphologies in which early-type AGB stars will often be characterized by an EDE, with complex flow patterns (Class 1), and the wind of late-type high mass-loss rate AGB stars will mainly be shaped by spiral structures (Class 3). The proposed evolutionary scheme for AGB wind morphologies can explain various AGB, post-AGB, and PNe phenomena, including for instance why post-AGB star binaries can have nonzero eccentricities (Decin et al. 2020). The binary scenario gets support from an analysis of the kinematic wind properties (Gottlieb et al. 2020). Moreover, it was shown that early-type AGB stars with a low mass-loss rate are prime candidates for detecting planets. This prediction aligns with the detection that Jupiter-sized companions reside in the near vicinity of the low mass-loss rate AGB stars L 2 Pup and R Dor (Kervella et al. 2016, Homan et al. 2018a).

In any case, regardless of the classification proposed by Decin et al. (2020), it should be emphasized that the same set of observations may on occasion be interpreted in different ways and may lead to the recognition of different categories. An immediate example might be that instead of the mass-loss rate, the wind acceleration is of greater importance for dictating the morphological outcome (see the discussion in Section S3.3 in Decin et al. 2020). When a system can be categorized in more than one way the question as to which category is better may depend on the particular phenomenon under study. The mapping is probably not simple, and the sample size needs to be significantly expanded before we can investigate the statistical aspects in detail.

\subsection{Statistics to probe the invisible}

The following logical proposition $(\models \mathcal{S}: A \vee \neg A)$ is a clear tautology of propositional logic as introduced by the great twentieth-century philosopher Ludwig Wittgenstein (1921) in his famous Tractatus. This logical tautology can be paraphrased by saying 'a star has either at least one companion, or a star lives its life alone'. I am of the view that this tautology might vanish in the foreseeable future with only the first part remaining — not only for massive stars, for which it has been established that binary interaction dominates their evolution (Sana et al. 2012); but also for the low and intermediate-mass stars, which are the focus of this review. It is quite obvious that this statement is inspired by one of the most significant developments in modern astronomy that we seem to find planets around stars almost everywhere we look. To assess this hypothesis, we consider another discipline in mathematics: statistics.

We therefore summarize the current state of knowledge concerning the occurrence rate of stellar and planetary companions around low and intermediate mass stars. To do so, I borrow heavily from Section S5 in the Supplementary Material of Decin et al. (2020) and references therein. For convenience, I here reproduce Table $\mathbf{1}$ which focusses on stars with initial mass, $M_{\text {ini }}$, between $0.8-8 \mathrm{M}_{\odot}$. It is well established that the binary fraction increases for higher initial masses, in this case for the more massive stars with $M_{\text {ini }} \gtrsim 8 \mathrm{M}_{\odot}$ that will evolve to the RSG stage, and that some fractions listed in Table $\mathbf{1}$ might be lower limits owing to limitations in detection techniques.

Table 1 is constructed on the arguments that we are looking at those binary systems for which the orbital separation (and hence orbital period $P_{\text {orb }}$ ) and the companion mass are such that hydrodynamical simulations indicate a perturbation of the smooth envelope structure. This leads to the conservative estimate of $\log P_{\text {orb }}$ (days) $<6.5$ and $\log P_{\text {orb }}$ (days $)>2.7$ (or 3 ) in the case of planets (or respectively, stars). Using these boundaries, we assess the 
Table 1 Summary of main-sequence (sub-)stellar multiplicity fraction.

\begin{tabular}{ll|cccc}
\hline & $\begin{array}{c}\text { Stellar } \\
\text { companions }\end{array}$ & $\begin{array}{c}\text { Brown } \\
\text { dwarfs }\end{array}$ & $\begin{array}{c}\text { Planets } \mathbf{M}>\mathbf{5} \mathbf{M}_{\text {Jup }} \\
\mathbf{a}-\mathbf{1 0} \mathbf{a u}\end{array}$ & $\mathbf{a = 1 0 - 1 0 0 ~ a u}$ \\
\hline $0.8<M_{\mathrm{ini}}<1.5 \mathrm{M}_{\odot}$ & $\mathrm{FGK}$ & $\sim 30-37 \%$ & $\sim 0.8 \%$ & $\sim 7 \%$ & $\sim 9 \%$ \\
$1.5<M_{\mathrm{ini}}<8 \mathrm{M}_{\odot}$ & $\mathrm{AB}$ & $\sim 52-60 \%$ & $\sim 0.8 \%$ & $\sim 7 \%$ & $\sim 40 \%$ \\
\hline Notes: The first and second columns give the main-sequence initial mass and related spectral type \\
on the main-sequence. Columns 3-6 list the (sub)-stellar multiplicity fraction for companions with \\
$\log P_{\text {orb }}$ (days) $<6.5$ and $\log P_{\text {orb }}$ (days) $>2.7$ (or 3) in the case of planets (respectively, stars).
\end{tabular}

main-sequence stellar and sub-stellar multiplicity factor (see Table 1 Moe \& Di Stefano (2017), Nielsen et al. (2019), Fulton \& Petigura (2018), Fulton (2019)). In a final step, one should account for the evolution of the (sub-)stellar binary fraction from the main-sequence to the AGB (and RSG) phase. A rough estimate is that the binary fraction will decrease by $\sim 10-20 \%$ as stars evolve toward and on the AGB/RSG phase (Decin et al. 2020).

A last realisation is adopted from population statistics. Based on stellar evolution models, it can be argued that the majority of AGB (and RSG) stars with a mass-loss rate above $10^{-7} \mathrm{M}_{\odot} \mathrm{yr}^{-1}$ are of mass above $1.5 \mathrm{M}_{\odot}$ (Decin et al. 2020). Stars of lower mass will only have a very short period in their AGB phase during which the mass-loss rate is greater than $10^{-7} \mathrm{M}_{\odot} \mathrm{yr}^{-1}$ before they transit into the $\mathrm{PN}$ phase, limiting their detection probability. Combining this outcome with Table 1 leads to the specific conjecture that most AGB stars with mass-loss rate above $10^{-7} \mathrm{M}_{\odot} \mathrm{yr}^{-1}$ have masses above $\sim 1.5 \mathrm{M}_{\odot}$ and hence have, on average, $\gtrsim 1$ companion(s) with masses above $\sim 5$ Jupiter masses. Hence (sub)stellar binary interaction is the prime wind shaping agent of the majority of cool ageing stars for which the mass-loss rate exceeds the nuclear burning rate $\left(\dot{M} \gtrsim 10^{-7} \mathrm{M}_{\odot} \mathrm{yr}^{-1}\right)$, in which case mass loss rules stellar evolution (Decin et al. 2020).

In conclusion, it took us some time to realize that roses flourish during the end stages of stellar evolution (see Figure 7), but resorting to one of the best-selling and most translated books ever published 'C'est le temps que tu as perdu pour ta rose, qui fait ta rose si importante' ('It's the time you spent on your rose that makes your rose so important'; de Saint-Exupéry 1943). The question then is how that rose impacts on our deterministic insight in the mass-loss rate of AGB and RSG stars.

\subsection{How can a companion change a stellar life}

Indeed, the stellar and planetary companions not only serve to beautify the stellar winds during the end stages a star's life, they can also have a direct impact on the star's evolution. For close binary systems, a stellar companion or a massive planet can enhance the massloss rate by depositing angular momentum into the envelope and by reducing the effective gravity of the mass-losing star. Stars that are born single or binary stars isolated from angular momentum deposition hence might suffer from a lower mass-loss rate than stars prone to angular momentum deposition (Sabach \& Soker 2018); for the example shown in Figure 8 this effect is a factor of $\sim 6$. This conclusion has a serious repercussion on any empirically retrieved mass-loss rate relation discussed in Section 2.2.4 Building on the discussion in Section 4.2 we conjecture that most empirically retrieved mass-loss rates yield mass-loss rate measures that are too high for application in single-star evolution models, since samples of stars will be flawed by a large fraction of stars that experience binary interaction (Gottlieb et al. 2020). This brings us back to one of the pitfalls of retrieval 
Type IIP supernovae: Type II supernovae can be further sub-classified into II-P (plateau light curves), II-L (linear decline light curves), IIn (narrow emission lines) and some peculiar events, generically labelled II-pec approaches outlined in Section 2.2, and the caution expressed there about unrecognised bias effects in sample selections and the elemental difference between correlation and causal effects.

There is a second no less fundamental problem with empirically retrieved mass-loss rates in the case of unrecognised binary interaction. For a mass-losing AGB/RSG star in a binary system, the material will have a directional preference towards the orbital plane, and an equatorial density enhancement (EDE) will form. The density contrast between the equator and the pole increases for smaller orbital distance, lower wind velocity and higher companion masses (El Mellah et al. 2020), with density contrasts up to an order of magnitude. However, dust mass-loss rates - which in a next step are converted to gas mass-loss rates - are most often derived from the analysis of near to mid-infrared spectral energy distributions (SEDs) that mainly trace warm dust residing close to the (primary) star, hence in the EDE (Decin et al. 2019). Therefore, the analysis of dust spectral features with a simplified 1D approach reflects the higher density in the EDE created by the binary interaction, but not the actual mass-loss rate which will be lower by up to an order of magnitude (Wiegert et al. 2020). This deduction amplifies the resolution of the previous paragraph, and leads to the inference that scientists modelling single-star evolution by applying empirically retrieved mass-loss rate formulae should be very cautious because the rates might be seriously overestimated. It is readily seen that this conclusion directly impacts any estimate of the chemical enrichment of the ISM by cool ageing stars. In what follows, I will make some suggestions and predictions that flow directly from the above conclusions.

The only prescriptions currently devoid of a binary-induced bias are the theoretical and — to a large extent - the semi-empirical mass-loss rate formulae (see Figure 5). However, these mass-loss rate prescriptions for AGB stars are only meant to describe the properties of Mira variables, while the only RSG theoretical mass-loss prescription from Kee et al. (2020) hinges on knowledge of the turbulent velocity which is barely constraint from observations and which does not yet account for any radial or tangential change. Supported by almost a billion CPU hours at HPC facilities for theoretical modelling purposes and the provision of specialised computing facilities for data analysis, several groups are now collaborating with the aim to provide the community with improved mass-loss rate prescriptions accounting for this $3 \mathrm{D}$ binary perspective.

4.3.1. The red supergiant problem. The preceding discussion will assist us in addressing the 'red supergiant problem', which concerns the question as to why the observed upper limit of $\sim 16 \mathrm{M}_{\odot}$ on the masses of Type IIP SN progenitors appears to be significantly lower than the maximum mass of $\sim 30 \mathrm{M}_{\odot}$ for stars expected to explode while they are red supergiants (Smartt et al. 2009). Numerous explanations have been considered including: a steeper initial mass function (Smartt et al. 2009); the loss of the loosely bound hydrogen envelope of the most massive RSG stars prior to core collapse (Yoon \& Cantiello 2010); the proposition that enhanced mass loss might limit the RSG mass to $\sim 20 \mathrm{M}_{\odot}$ (Groh et al. 2013); or the fact that massive stars collapse to black holes with optically dark or faint 'failed' SNe (Woosley \& Heger 2012). Another direction was to argue that for stars whose mass is greater than $\sim 15 \mathrm{M}_{\odot}$, the mass-loss rate should be higher than that currently considered in stellar evolution codes (Ekström et al. 2012); as cause for this increase in massloss rate, it was suggested that some of the most external layers of the stellar envelope might 
exceed the Eddington luminosity. Each of these explanations is grounded on uncertainties in the theoretical models, with the latter proposal of Ekström et al. (2012) seeming mutually incompatible with the conjecture formulated at the start of Section 4.3

However, the puzzle that red supergiants with masses $\sim 16-30 \mathrm{M}_{\odot}$ have not been identified as progenitors of Type IIP supernovae can also be examined from the perspective of retrieval analyses. An insufficient circumstellar dust correction for the pre-SN mass loss could bias the progenitor mass estimates to lower values (Walmswell \& Eldridge 2012). Although the SED analysis of Walmswell \& Eldridge (2012) was criticized by Kochanek et al. (2012), the underlying rationale can be reviewed in the light of the scientific discourse embarked upon at the start of Section 4.3. Admittedly, the following discussion is narrowly confined to this one characteristic of retrieval modelling, and other potentially influential characteristics are not considered.

A first point of consideration deals with the binary hypothesis, and the well-established issue that the binary fraction increases with stellar mass. The larger the stellar mass of the primary star, the larger the fraction of companions that will inspiral owing to the larger gravitational attraction by the primary star (Decin et al. 2020). For a binary system with a decreasing orbital distance, the EDE will get more pronounced. Higher densities in the EDE promote the formation of (crystalline) dust grains (Decin et al. 2019). Hence, it is to be expected that stars of higher initial mass can have a higher dust mass in their circumstellar envelope, so the circumstellar dust correction is weighted higher in the final estimate of the pre-SN mass loss. A second manner of reasoning touches upon the issue of $3 \mathrm{D}$ clumps. Following the argument of Schwarzschild (1975), the size of the convection bubbles for stars of higher mass (and larger radius) will be larger. Using the same diameter-to-depth ratio of 3 as Schwarzschild (1975), this implies that the depth — and corresponding dust optical depth - might be larger for more massive stars so the impact of a proper circumstellar dust correction is larger. These assertions permit the extreme hypothesis that the red supergiant problem is potentially not so severe as currently considered, and the real solution can only be formulated under the condition of a detailed 3D analysis of the pre-SN mass loss. Of course this is not a Solomon-like resolution, but only one step in a holistic approach of tackling this dilemma. Recent studies even argue that the upper limit cut-off is likely to be higher and has large uncertainties $\left(M=19_{-2}^{+4} \mathrm{M}_{\odot}\right)$, implying that the statistical significance of the RSG problem is less than $2 \sigma$ (Davies \& Beasor 2020ba).

4.3.2. Survival rate of (exo)planets. The future of our own planet, the Earth, seems far from smooth sailing. Cogent modelling by Schröder \& Smith (2008) suggests that the Earth will be swallowed by the time that the Sun is near its tip of the red giant branch (RGB) evolution, $\sim 7.6$ Gyr from now. At that moment, the solar radius will reach $\sim 1.2$ au, while the Earth's orbit will hardly ever exceed 1 au by a significant amount. This is caused by a competition between orbital widening induced by the solar RGB wind reducing the Sun's mass, and orbital tightening owing to dynamical drag with the lower chromosphere and to orbital angular momentum loss due to tidal interaction with the giant Sun. Tidal interaction is dominant so the planet Earth cannot escape engulfment. Although not all parameters are invariably rigorously defined - in particular the tidal friction coefficient it seems that doomsday is unavoidable. There is a future beyond the Sun's RGB evolution only for planets whose current orbital separation is greater than $\sim 1.15 \mathrm{au}$.

A larger RGB mass-loss rate would delay the engulfment and lead to smaller orbital separations for planets still able to survive. In their modelling approach, Schröder \& Smith
Eddington

luminosity: maximum luminosity a star can achieve for there to be a balance between the radiation force acting outward and the gravitational force acting inward

Solomon-like resolution: named after King Solomon of Israel (Hebrew Bible), a famous practitioner of dispute resolution

RGB phase: follows the main-sequence phase; stars have an inert helium core surrounded by a shell of hydrogen fusion 
(2008) used an adapted form of Reimers' law (1975) to describe the cool wind - not driven by dust but presumably by (magneto)acoustic processes - which was physically motivated by a consideration of global chromospheric properties and wind energy requirements

$$
\dot{M}=4 \times 10^{-13} \eta_{\mathrm{SC}} \frac{\mathrm{L}_{\star} \mathrm{R}_{\star}}{\mathrm{M}_{\star}}\left(\frac{T_{\mathrm{eff}}}{4000 \mathrm{~K}}\right)^{3.5}\left(1+\frac{g_{\odot}}{4300 g_{\star}}\right),
$$

with $\eta_{\mathrm{SC}}=0.2$ and $g_{\odot}$ the solar surface gravitational acceleration. Following Eq. 20, the mass-loss rate of the giant Sun would be around $4 \times 10^{-8} \mathrm{M}_{\odot} \mathrm{yr}^{-1}$ at the tip of the RGB. The feeble RGB mass loss is difficult to constrain from observations. The first detection of rotational CO line emission arising from an RGB wind was announced in 2014, the derived mass-loss rate is around a few $10^{-9} \mathrm{M}_{\odot} \mathrm{yr}^{-1}$ (Groenewegen 2014). A promising way to measure the RGB mass loss is to obtain a difference in stellar mass between two points in its evolution, and use this to determine a scaling parameter such as $\eta$ in Eq. 1 or $\eta_{\mathrm{SC}}$ in Eq. 20. (McDonald \& Zijlstra|2015). This can be achieved in globular clusters, where one can probe the individual stars with accurately known distances, metallicities, abundances and ages. Using 56 well-studied global clusters, McDonald \& Zijlstra (2015) derived as median values $\eta=0.477 \pm 0.070$ and $\eta_{\mathrm{SC}}=0.172 \pm 0.024$, with very little metallicity dependence. This provides support to a Reimers-like law being a good RGB mass-loss model and to the model predictions of Schröder \& Smith (2008).

Given the conjecture that AGB mass-loss rates for single stars or stars isolated from angular momentum deposition might be overestimated, the follow-up question then deals with the impact of that conjecture on the survival rate of planets. At first sight, this question might seem quite semantic, given the current lack of directly imaged planets in the close vicinity of cool ageing stars and remembering the critical remark on that matter (see Section 4.1. But to quote the old maxim: 'Absence of evidence is not evidence of absence'. Given the large amount of exoplanets currently detected and expected to be present in the Universe, and our current knowledge of stellar and planetary evolution, this inquiry on the survival potential of (exo)planets gets more substantial. For a reduced AGB/RSG mass-loss rate the predictions are, however, not at all auspicious since the change of orbital separation, $\mathrm{d} a / \mathrm{d} t$, scales with the mass-loss rate. The lower mass-loss rate implies a lower rate of orbital widening, if occurring, and hence more (exo)planets experiencing a catastrophic encounter with their giant mother star due to tidal interaction. Therefore escaping the doomsday scenario is more challenging than previously suggested.

\section{The 3D life of molecules and dust grains}

To comprehend the essence of mass loss requires an understanding of the driving forces that dictate the nature of the phenomenon. Delving into the why question will provide feedback to the how question, and vice-versa. Our view of the essential processes has gradually been enriched thanks to the enormous capacities of modern telescopes and space observatories designed for optical, IR, and microwave investigations of cool astronomical objects. Moreover, a new window for exploring dust formed in stellar outflows commenced in 1987 with the detection of small particles in the matrix material of certain meteorites which originated from stars whose lives ended before the formation of our Solar system: the presolar grains (Nittler \& Ciesla 2016). Long before this detection, Cameron (1973) speculated about 'Interstellar grains in museums?' and concluded that primitive carbonaceous chondrites may harbour presolar grains, but it was Lewis et al. (1987) who stated 'Interstellar dust contains 
diamonds' in the final phrase in the abstract of their paper in Nature. Before you consider any space exploration, the diamonds were tiny — i.e., only about $\sim 10 \AA$.

This is where we reach the other spectrum of astrophysics, the regime where the small pieces make up the big picture. These small pieces seldom bear any resemblance with smooth spherical particles, not even for the micrometer-sized dust grains (see Figure 7 ). For chemists, micrometer-sized dust grains are 'macroscopic'. Even when staying within the realm of solid-state physics, it is well established that further size reduction from the macroscopic to length scales of only a few nanometers can lead to dramatic changes in a material's properties, including its atomic ordering, quantum, and surface effects (e.g., Lamiel-Garcia et al. 2017, Gobrecht et al. 2017). Wondering then how solid-state species form, we enter the world of atoms and molecules which undergo a sequence of collisional association and recombination reactions to form polyatomic molecules of ever-increasing size, the gas-phase clusters (see Figure 7 and Supplemental Text). Under favourable conditions the clusters condense, and form the first dust seeds consisting of 10 to several hundred atoms. The seed nuclei become the substrate to which molecules are added, resulting in further growth of the condensed phase which eventually can reach the macroscopic regime. By the end of this process, the macroscopic dust species involve the accumulation of typically $10^{6}-10^{9}$ atoms in a single grain.

\subsection{Molecules and dust grains identified in stellar outflows}

5.1.1. Molecules. Observatories that operate in the micrometer and millimeter-wave bands such as the Infrared Space Observatory (ISO), the James Clerk Maxwell Telescope (JCMT), the IRAM-30m telescope, the Plateau de Bure Interferometer (PdBI), and ALMA have boosted the detection of cosmic molecules via their rotational and vibration-rotation spectroscopic fingerprints. The wavelength resolution of most telescopes in the mid and near-IR is moderate, $\Delta \lambda / \lambda \sim 10^{-4}$, compared with gas-phase spectra whose frequencies can be determined to an accuracy of about 1 part in $10^{7}$ or better in narrow line sources with heterodyne receivers on radio telescopes. In his excellent review, Olofsson (2005) provided a table listing the detection of 63 molecules in AGB winds. Table 2 gives an update fifteen years later: currently 105 molecules have been detected in stellar outflows. This is roughly $50 \%$ of all molecules currently discovered in outer space: the extensive review by McGuire (2018) lists 204 molecules, to which 6 other ones can be included (MgCCH, $\mathrm{MgC}_{3} \mathrm{~N}, \mathrm{MgC}_{4} \mathrm{H}, \mathrm{CaNC}$ and potential detections of NCCP and $\mathrm{FeO}$; Agúndez et al. 2014 , Cernicharo et al. 2019a b, Decin et al. 2018a). I opted to include in Table 2 the polycyclic aromatic hydrocarbon molecules (PAH) since their characteristic $\mathrm{C}-\mathrm{H}$ and $\mathrm{C}-\mathrm{C}$ stretching and bending modes have been detected in AGB stars (Boersma et al. 2006, Smolders et al. 2010), although the precise carriers have not been determined.

In addition, there is the obvious difference between being and seeing. Given the current detections, our knowledge of quantum-chemical selection rules, reaction kinetics, and the thermodynamical wind properties, we have no doubt about the presence of some molecular species, but they remain hidden from our telescopes. The most obvious and important example is molecular hydrogen, $\mathrm{H}_{2}$, the most abundant molecule in stellar winds. This nonpolar light molecule has no permanent electric dipole moment and is not easily observed under the conditions prevalent in AGB CSEs. However, $\mathrm{H}_{2}$ is detected in $2 \mu \mathrm{m}$ spectra of AGB variables (Hinkle et al. 2000), but it is not always straightforward to determine whether an infrared line has a circumstellar origin or whether it emerges from the (extended)
Reaction kinetics: or chemical kinetics, dealing with understanding the rates of chemical reactions 


\begin{tabular}{|c|c|c|c|c|c|c|}
\hline \multirow[t]{5}{*}{ 2-atoms } & $\mathrm{AlCl}$ & $\mathrm{AlF}$ & $\mathrm{AlO}$ & $\mathrm{C}_{2}$ & CN & $\mathrm{CO}$ \\
\hline & $\mathrm{CP}$ & CS & $\mathrm{FeO}(?)$ & $\mathrm{HCl}$ & $\mathrm{HF}$ & $\mathrm{KCl}$ \\
\hline & $\mathrm{NO}$ & NS & $\mathrm{NaCl}$ & $\mathrm{OH}$ & PN & $\mathrm{PO}$ \\
\hline & SO & $\mathrm{SiC}$ & $\mathrm{SiN}$ & $\mathrm{SiO}$ & $\mathrm{SiS}$ & $\mathrm{TiO}$ \\
\hline & $\mathrm{VO}$ & & & & & \\
\hline \multirow[t]{5}{*}{ 3-atoms } & AlNC & $\mathrm{AlOH}$ & $\mathrm{C}_{2} \mathrm{H}$ & $\mathrm{C}_{2} \mathrm{~S}$ & $\mathrm{C}_{3}$ & $\mathrm{CCN}$ \\
\hline & $\mathrm{CCP}$ & $\mathrm{CO}_{2}$ & $\mathrm{CaNC}$ & $\mathrm{FeCN}$ & $\mathrm{H}_{2} \mathrm{O}$ & $\mathrm{H}_{2} \mathrm{~S}$ \\
\hline & $\mathrm{HCN}$ & $\mathrm{HCP}$ & $\mathrm{HNC}$ & $\mathrm{KCN}$ & $\mathrm{MgCN}$ & $\mathrm{MgNC}$ \\
\hline & $\mathrm{NaCN}$ & $\mathrm{SO}_{2}$ & $\mathrm{SiC}_{2}$ & $\mathrm{SiCN}$ & $\mathrm{SiCSi}$ & SiNC \\
\hline & $\begin{array}{l}\mathrm{TiO}_{2} \\
c-\mathrm{C}_{3} \mathrm{H}\end{array}$ & $l-\mathrm{C}_{3} \mathrm{H}$ & $\mathrm{C}_{2} \mathrm{H}_{2}$ & $\mathrm{C}_{3} \mathrm{~N}$ & $\mathrm{C}_{3} \mathrm{O}$ & $\mathrm{C}_{3} \mathrm{~S}$ \\
\hline \multirow{2}{*}{ 4-atoms } & $\mathrm{H}_{2} \mathrm{CO}$ & $\mathrm{H}_{2} \mathrm{CS}$ & $\mathrm{HC}_{2} \mathrm{~N}$ & $\mathrm{HMgNC}$ & $\mathrm{MgCCH}$ & $\operatorname{NCCP}(?)$ \\
\hline & $\mathrm{NH}_{3}$ & $\mathrm{PH}_{3}$ & $\mathrm{SiC}_{3}$ & & & \\
\hline \multirow[t]{2}{*}{ 5-atoms } & $c-\mathrm{C}_{3} \mathrm{H}_{2}$ & $\mathrm{C}_{4} \mathrm{H}$ & $\mathrm{C}_{5}$ & $\mathrm{CH}_{2} \mathrm{CN}$ & $\mathrm{CH}_{2} \mathrm{NH}$ & $\mathrm{CH}_{4}$ \\
\hline & $\begin{array}{l}\mathrm{H}_{2} \mathrm{CCC} \\
\mathrm{SiH}_{4}\end{array}$ & HCCCN & HCCNC & $\mathrm{HNC}_{3}$ & $\mathrm{MgC}_{3} \mathrm{~N}$ & $\mathrm{SiC}_{4}$ \\
\hline \multirow[t]{2}{*}{ 6-atoms } & $\mathrm{C}_{2} \mathrm{H}_{4}$ & $\mathrm{C}_{5} \mathrm{H}$ & $\mathrm{C}_{5} \mathrm{~N}$ & $\mathrm{C}_{5} \mathrm{~S}$ & $\mathrm{CH}_{3} \mathrm{CN}$ & $\mathrm{H}_{2} \mathrm{CCCC}$ \\
\hline & $\mathrm{HC}_{4} \mathrm{~N}$ & $\mathrm{MgC}_{4} \mathrm{H}$ & $\mathrm{SiH}_{3} \mathrm{CN}$ & & & \\
\hline \multirow[t]{2}{*}{$\geq 7$-atoms } & $\mathrm{C}_{6} \mathrm{H}$ & $\mathrm{C}_{7} \mathrm{H}$ & $\mathrm{C}_{8} \mathrm{H}$ & $\mathrm{CH}_{2} \mathrm{CHCN}$ & $\mathrm{CH}_{3} \mathrm{CCH}$ & $\mathrm{CH}_{3} \mathrm{SiH}_{3}$ \\
\hline & $\mathrm{H}_{2} \mathrm{C}_{6}$ & $\mathrm{HC}_{5} \mathrm{~N}$ & $\mathrm{HC}_{7} \mathrm{~N}$ & $\mathrm{HC}_{9} \mathrm{~N}$ & PAH & \\
\hline Ions & $\begin{array}{l}\mathrm{C}_{3} \mathrm{~N}^{-} \\
\mathrm{HCO}^{+}\end{array}$ & $\mathrm{C}_{4} \mathrm{H}^{-}$ & $\mathrm{C}_{5} \mathrm{~N}^{-}$ & $\mathrm{C}_{6} \mathrm{H}^{-}$ & $\mathrm{C}_{8} \mathrm{H}^{-}$ & $\mathrm{CN}^{-}$ \\
\hline
\end{tabular}

Notes: (?) indicates a tentative identification

atmosphere. The same argument holds for other molecules such as $\mathrm{CH}$ (Matsuura et al. 2007).

Of the 105 molecules listed in Table 2, only 27 do not contain carbon and most are diatomic species. This is due to the reactive nature and unique bonding properties of the carbon atom, but also because the nearest $(D \sim 150 \mathrm{pc})$ and best known carbon star, CW Leo, has a high mass-loss rate $\left(\dot{M} \sim 1.5 \times 10^{-5} \mathrm{M}_{\odot} \mathrm{yr}^{-1}\right.$, De Beck et al. 2012 , Cernicharo et al. 2015). Just for comparison, the nearest O-rich AGB star R Dor resides at a distance of $\sim 59 \mathrm{pc}$ and has a mass-loss rate of only $\sim 1.5 \times 10^{-7} \mathrm{M}_{\odot} \mathrm{yr}^{-1}$ (Danilovich et al. 2016), while the O-rich supergiant VY CMa has an enormous mass-loss rate of $\sim 2 \times 10^{-4} \mathrm{M}_{\odot} \mathrm{yr}^{-1}$, but resides at a distance of $\sim 1150$ pc (Decin et al. 2006, Zhang et al. 2012).

In all CSEs the dominant molecule apart from $\mathrm{H}_{2}$ is $\mathrm{CO}$, which forms at local thermodynamic equilibrium (LTE) in the stellar photosphere and consumes all of the remaining atomic $\mathrm{C}$ or $\mathrm{O}$ - i.e., whichever is the least abundant of the two. (See the sidebar titled Carbon- and oxygen-rich cool stars.) For a long time, it was therefore thought that O-rich winds are very deficient in other carbon-bearing molecules, while C-rich ones are deficient in other oxygen-bearing molecules, but this picture has gradually been amended. First of all, there was the detection of HCN, HNC, and CS in O-rich CSEs which were explained on the assumption of an active photochemistry in the outer CSE layers owing to the penetration of harsh interstellar UV photons (Bujarrabal et al. 1994). While this process can explain the formation of 'atypical' molecules in the outer envelope, it cannot resolve the unanticipated detection of $\mathrm{CO}_{2}$ in the inner wind of O-rich AGB stars and $\mathrm{H}_{2} \mathrm{O}, \mathrm{OH}, \mathrm{H}_{2} \mathrm{CO}$, and $\mathrm{SiO}$ in carbon-rich winds (Justtanont et al. 1998, Ryde et al. 1998, Melnick et al. 2001, 
Ford et al. 2003, 2004, Schöier et al. 2006, Decin et al. 2008, Schöier et al. 2013, Velilla Prieto et al. 2015). Little by little, it became clear that we should abandon the convenient idea of LTE, and need to acknowledge that the chemistry is more complex than inferred by the assumption of thermodynamic equilibrium and instead is best described as 'nonequilibrium chemistry'. I say 'little by little', since that change in mindset didn't happen silently and overnight. It was not that there was a disregard of unwelcome evidence, but non-equilibrium calculations imply solving the full set of rate equations for the detailed reaction kinetics in the rapidly expanding and cooling gas. These calculations are not only CPU expensive, the challenge here is also to know the rate coefficients, which commonly depend on the temperature and often also on the gas density or on external sources such as the ISM radiation field or cosmic rays. Not only can chemical reactions relevant for astrophysics rarely be extracted from industrial resources, in addition the gas number densities in CSEs are generally lower than in the laboratory by 10 decades. One should also realize that only $\sim 15 \%$ of the reaction rates listed in astrochemical databases are experimentally obtained at room temperature $(\sim 300 \mathrm{~K})$, only $\sim 2 \%$ have rate constants at temperatures below $200 \mathrm{~K}$ and less than $0.5 \%$ at temperatures below $100 \mathrm{~K}$. For some reaction rates, the results of theoretical quantum-chemical calculations are available. However, the complexity of the electronic structures often complicates a reliable theoretical prediction of the rate coefficients. Accurate rates are in particular critical for the rate-limiting steps (see Section 5.3.1.

Photo-induced processes in the outer CSE are not the only cause for out-of-equilibrium chemistry. In general, the low particle densities in CSEs imply that reaction conditions can change considerably before a reaction has run to completion, a particular example is the extended atmosphere which is subject to pulsation-induced shocks (Cherchneff 2011, Gobrecht et al. 2016). In addition, the material is subject to chromospheric UV fields (notably for RSG stars) and ionizing radiation from decaying radioactive nuclei. We now think that photo-induced processes are not only relevant in dictating the outer-envelope chemistry: given the fact that contemporary observations reveal the envelopes to be nonhomogeneous (see Section 3), recent theoretical models incorporating the emergence of 3D clumps indicate an enhanced penetration depth of interstellar photons resulting in a vast difference in predicted molecular abundance structures compared with the smooth envelope case (Agúndez et al. 2010, Van de Sande et al. 2018b, Van de Sande \& Millar 2019).

In addition, we can get an extra glimpse of the molecular content of the winds of AGB stars, by studying the circumstellar environment of their immediate successors, the young post-AGB stars, where the increased UV flux and the presence of shocks can affect the chemistry. Good examples include the envelopes of the C-rich objects AFGL 618 and

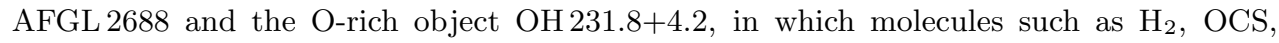
$\mathrm{HNCO}, \mathrm{HNCS}, \mathrm{CH}_{3} \mathrm{OH}, \mathrm{H}_{3} \mathrm{O}^{+}, \mathrm{SO}^{+}, \mathrm{CO}^{+}, \mathrm{CH}^{+}, \mathrm{N}_{2} \mathrm{H}^{+}, \mathrm{H}_{2} \mathrm{CO}, \mathrm{HC}_{4} \mathrm{H}, \mathrm{HC}_{6} \mathrm{H}, \mathrm{CH}_{3} \mathrm{C}_{2} \mathrm{H}$, $\mathrm{CH}_{3} \mathrm{C}_{4} \mathrm{H}$, and $\mathrm{C}_{6} \mathrm{H}_{6}$ have been detected.

These molecules and atoms form the building blocks of the solid-state species which can form if density and temperature are of the right order for dust formation to take place. The process of solidification is modelled under the common interpretive framework of nucleation, a process during which the nano-to-microscale transition of nuclei form; see also Section 5.3 (See the sidebar titled Nucleation.)

5.1.2. Condensates. Condensates are characterised by their mineralogical structure (monocrystalline, polycrystalline, or amorphous), their chemical composition (homoge- 


\section{Nucleation}

Gas does not spontaneously go over to the solid bulk phase because there exists an energy barrier between the metastable and stable phases that makes a global phase change highly improbable. An energetically more favourable pathway is the formation of nano-to-microscale density transition nuclei, called the cluster model of the nucleation process. During this process, atoms and molecules undergo a sequence of collisional association reactions to form polyatomic molecules of ever increasing size. Clusters are generally represented by the symbol $M_{N}$, which is an assemblage of a (small) number $N$ of atoms or molecules, denoted here as monomer $M$. Obviously, the term small is ambiguous, and distinguishing between a small gas-phase cluster and a larger particle can be difficult. A common practice is to define a small cluster as a system that still has a discrete electronic structure (as in an atom), and therefore differs from a larger system which has electronic bands. The properties of clusters change drastically with their size including optical, electronic, magnetic and chemical properties. Generally, nucleation is a very inefficient process and only a small fraction of the collisions between the gas phase atom or molecule and a small cluster will lead to cluster growth.

neous, but most often heterogeneous and layered), and their geometric shape. In contrast to molecules, the analysis of IR dust spectral features does not always provide sufficient insight into the mineralogical, chemical, and geometric properties. This stems from the fact that dust spectral features often originate in radiatively excited functional groups of atoms constituting the grain, such as the stretching vibration of the Si-O bond (around $9.5 \mu \mathrm{m}$ ) and the bending vibrations of the O-Si-O group (around $18.5 \mu \mathrm{m}$ ) within the $\mathrm{SiO}_{4}$ tetrahedron of silicates. As such, it is often impossible to unequivocally determine the exact grain properties from the analysis of IR spectra since, for example, two different amorphous silicate-type grains can each display the broad emission pattern in their mass absorption coefficient around 9.5 and $18.5 \mu \mathrm{m}$ (Jaeger et al. 1998, Molster et al. 2002a). This is also the reason why the PAH molecules are sometimes analysed in a similar way as dust species, since also for these macroscopic PAHs we witness the behaviour of the functional $\mathrm{C}-\mathrm{H}$ and C-C groups.

In particular the Infrared Space Observatory was ground-breaking for detecting new dust species (Tielens et al. 1998, Waters et al.|2000, Henning|2010); an overview of the dust grains identified in the winds of AGB and RSG stars can be found in Table 3. Instruments such as the ESO VLTI-MIDI and VLT-SPHERE interferometers were crucial for determining the location of the grains (Ohnaka et al. 2006, Norris et al. 2012, Karovicova et al. 2013). In this context, a very interesting yet unexpected discovery was the spectroscopic identification of pure crystalline silicate grains (pure forsterite, $\mathrm{Mg}_{2} \mathrm{SiO}_{4}$, and enstatite, $\mathrm{MgSiO}_{3}$, the $\mathrm{Mg}$ rich end members of olivine and pyroxene; Molster et al. 2002a). Given an expanding and cooling envelope, grains are predicted to be amorphous and additional processes were sought for a further crystallisation of the grains (see also Section 5.2 . Some low-temperature crystallization process of unknown origin acting in circumstellar disks has been proposed (Molster et al. 1999b). However, our recent finding that the majority of cool ageing stars with a measurable mass-loss rate might not live their lives alone, but have a companion offers an alternative explanation (Section 4): in O-rich winds with low wind acceleration properties the companion can quite easily induce the formation of an equatorial density enhancement (El Mellah et al. 2020), which offers the perfect conditions for amorphous 
grains to be annealed into crystalline minerals owing to shock formation with an associated increase in temperature in the orbital plane (Decin et al. 2019).

What is really fascinating is that physical samples of these dust grains can be assembled here on Earth: these microscopic stardust grains are found in meteorites (see Figure 7). After their journey through the ISM, these grains survived the formation of the Solar System, where they were trapped inside asteroids, the parent bodies of the meteorites. These presolar grains have isotopic compositions indicating an AGB or RSG origin (Lodders \& Amari 2005, Nittler et al. 2008, Davis 2011, Nittler \& Ciesla 2016). A common property is that they are chemically stable and for this reason have survived their journey to Earth. The grain's morphology, mineralogy, and composition reflect the formation conditions in the CSE, and hence complement the studies of the IR spectral bands. The cosmochemical studies of these extraterrestrial rocks even allowed the identification of AGB and RSG dust grains that escape identification via infrared spectral features (see bottom part of Table 3 . Typical grain sizes range between a few nanometer up to $1 \mu \mathrm{m}$ (Goderis et al. 2016). One should realize, however, that the laboratory techniques induce a bias in species detected due to the fact that some species might be dissolved out during the preparation process. In addition, smaller grains are more difficult to detect, resulting in a grain size bias.

Table 3 Condensates identified in the winds of cool evolved stars

\begin{tabular}{|c|c|c|c|}
\hline Species & Formula & Species & Formula \\
\hline Alumina & $\mathrm{Al}_{2} \mathrm{O}_{3}$ & Diopside & $\mathrm{MgCaSi}_{2} \mathrm{O}_{6}$ \\
\hline Olivine & $\mathrm{Mg}_{(2-2 x)} \mathrm{Fe}_{2 x} \mathrm{SiO}_{4}$ & Water ice & $\mathrm{H}_{2} \mathrm{O}$ \\
\hline Pyroxene & $\mathrm{Mg}_{(1-x)} \mathrm{Fe}_{x} \mathrm{SiO}_{3}$ & Metallic Fe & $\mathrm{Fe}$ \\
\hline Melilite & $\mathrm{Ca}_{2} \mathrm{Al}_{2} \mathrm{SiO}_{7}$ & Carbon & $\mathrm{C}$ \\
\hline Magnesiowüstite & $\mathrm{Mg}_{x} \mathrm{Fe}_{(1-x)} \mathrm{O}$ & Silicon Carbide & $\mathrm{SiC}$ \\
\hline Spinel & $\mathrm{MgAl}_{2} \mathrm{O}_{4}$ & Magnesium sulfide & $\mathrm{MgS}$ \\
\hline Silica & $\mathrm{SiO}_{2}$ & Hibonite & $\mathrm{CaAl}_{12} \mathrm{O}_{19}$ \\
\hline Titanium oxide & $\mathrm{TiO}_{2}$ & Titanium Carbide & $\mathrm{TiC}$ \\
\hline
\end{tabular}

AGB/RSG winds. More detailed information can be found in the Supplemental Tables $4-5$

\subsection{Challenging the why question}

The wealth of observations has guided the theoretical models and conveys the principles to which the models must adhere. The ISO observations of circumstellar dust around AGB stars have been interpreted within the context of thermodynamic equilibrium condensation sequences (Onaka et al. 1989 , Tielens et al. 1998). Gearing the discussion toward oxygenrich environments, the silicate thermodynamic condensation sequence predicts two branches to occur (Grossman \& Larimer 1974, Tielens 1990). The condensation sequence starts with that of $\mathrm{Al}_{2} \mathrm{O}_{3}$ (around $1700 \mathrm{~K}$ ). Gas-solid reactions lead to the formation of the first silicates, melilite $\left(\mathrm{Ca}_{2} \mathrm{Al}_{2} \mathrm{SiO}_{7}\right)$ and diopside $\left(\mathrm{CaMgSi}_{2} \mathrm{O}_{6}\right)$ around $1500 \mathrm{~K}$. Most of the silicon will condense out in the form of pure $\mathrm{Mg}$-rich silicates, first forsterite $\left(\mathrm{Mg}_{2} \mathrm{SiO}_{4}\right.$, around $1440 \mathrm{~K}$ ) and later enstatite $\left(\mathrm{MgSiO}_{3}\right)$. Finally, reactions with gaseous iron will convert some enstatite into fayalite $\left(\mathrm{Fe}_{2} \mathrm{SiO}_{4}\right)$ at about $1100 \mathrm{~K}$.

Observations indicate a very low $\mathrm{Fe} / \mathrm{Mg}$ content of crystalline silicates $(\mathrm{Fe}<10 \%$; Molster et al. 1999a). This can be explained in a scenario proposed by Tielens et al. (1998) in which crystalline Mg-rich silicates form close to the photosphere. These grains are thermally 
Glass temperature: above the glass temperature atoms in an amorphous solid are very mobile (i.e. low viscosity) and can rearrange themselves in a crystalline lattice coupled to the gas via collisions and attain temperatures well above the glass temperature (of $\sim 1050 \mathrm{~K}$ ), resulting in a crystalline structure. Upon the expansion and cooling of the gas, it becomes possible for Fe to be gradually incorporated in the silicate grains. The inclusion of Fe results in a considerable increase in the near-IR absorption coefficient so that just enough Fe can be incorporated for the radiative temperature of the grain to remain below the sublimation temperature of around $800-1100 \mathrm{~K}$ with an Fe-inclusion of around $20 \%$. However, at these low temperatures the Fe-rich lattice cannot reach its energetically most favourable structure leading to the formation of amorphous Fe-rich silicates. The Fe-rich silicates efficiently absorb the stellar light in the near-IR so that the radiation pressure on the grains becomes large enough to initiate a stellar wind. The scenario proposed by Tielens et al. (1998) rests on an important conjecture, namely that efficient dust nucleation and growth actually take place close to the stellar photosphere. As discussed by Tielens (1990) this is far from obvious not only since the densities have to be high $\left(n \gtrsim 10^{13} \mathrm{~cm}^{-3}\right)$ for efficient nucleation, but also since there seems to be a lack of stable monomers.

Given the observations, thermodynamics seemed to be well obeyed, if one accounts for the 'freezing-out' of chemical reactions which occurs when the density and temperature are too low and chemical reactions cease (Tielens et al. 1998). In this way, the 'macroscopic' world of dust grains was handled under the convenient assumption of thermodynamic equilibrium. If so, one should not care about the exact pathway of dust condensation since the most stable condensates will form under the general temperature-pressure conditions and relevant element mixtures. One can compare it with the analogy of a ball rolling down a rather smooth - hill: the ball will come at rest in the valley independent of the path taken. So, the thermodynamic equilibrium principles provided an answer to the why question.

A striking disruption of this comfortable interpretation occurred in 2006, when Woitke applied an elegant deduction - by essentially solving Eq. 4. and Eq. 6 - to prove that the qualitative scenario proposed by Tielens et al. (1998) for the wind driving in O-rich AGB stars actually does not hold (Woitke 2006b). In addition, through the adoption of detailed RHD models, Woitke showed that, in fact, two dust layers are formed, an almost pure glassy $\mathrm{Al}_{2} \mathrm{O}_{3}$ layer close to the star, and a more opaque Fe-poor Mg-Fe-silicate layer further out. However, since only a small fraction of Fe can be incorporated in the silicate grains, because otherwise they become too hot, almost no mass loss occurred $\left(\dot{M} \lesssim 10^{-10} \mathrm{M}_{\odot} \mathrm{yr}^{-1}\right)$. This paper not only re-opened the quest for the driving mechanism of O-rich AGB winds, but also touches on the fundamental why question.

Various solutions have been offered to solve this dilemma. Höfner \& Andersen (2007) provided an unorthodox suggestion - their words - involving the formation of both carbon and silicate grains. This scenario implies departures from chemical equilibrium (CE), since a fraction of the carbon is not bound in gaseous $\mathrm{CO}$, but available for grain formation. One year later, Höfner (2008) provided an alternative mechanism in which the Fe-free grains can grow to sizes large enough $(\sim 200 \mathrm{~nm}-1 \mu \mathrm{m})$ close to the star for photon scattering to compensate for their low near-infrared absorption cross-sections and to trigger the onset of a stellar wind. Recent VLT-NACO and SPHERE data support the presence of large transparent grains $(\sim 0.3 \mu \mathrm{m})$ at a distance of $\sim 0.5 \mathrm{R}_{\star}$ in some AGB stars (Norris et al. 2012, Khouri et al. 2016), but these data cannot pinpoint the chemical composition of the grains. The current line of reasoning stipulates that $\mathrm{Al}_{2} \mathrm{O}_{3}$ grains can grow around $\sim 0.5 \mathrm{R}_{\star}$ from the star and reside there in a gravitationally bound dust shell (Khouri et al. 2015, Höfner et al. 2016, Höfner \& Freytag 2019). Once the temperature gets below $\sim 1200 \mathrm{~K}$ (around $4 R_{\star}$ ), silicates can form as a mantle around the aluminium-oxide cores. For grain 
sizes above $\sim 100 \mathrm{~nm}$, a dust-driven outflow can be expected owing to the operation of scattering.

Each of the proposed solutions is, however, a 'macroscopic' solution and bypasses the question of the molecular formation routes towards the predicted grain types. Although Höfner \& Andersen (2007) suggested a non-equilibrium formation route for carbon grains, they did not present a detailed non-CE study, and the abundance of available carbon was a parameter in the models. In the same vein, most modern radiation hydrodynamic wind models for O-rich AGB winds assume the first dust seeds are present, after which further growth to micrometer-sized dust grains can take place (Höfner \& Olofsson|2018). Hence, the conjecture posed by Tielens (1990) is still open some thirty years later: we still assume in the models that efficient dust nucleation occurs close to the star, although we now acknowledge the shortcomings of thermodynamic equilibrium to understand dust nucleation.

Leaving behind the comfortable landscape of thermodynamic equilibrium, one enters a new gigantic playground in which the rules of the game are not always very clear, and sometimes even undefined. Given the earlier discussion on the challenges of gas-phase chemical kinetics (Section 5.1), it is to be acknowledged that a proper description of dust nucleation is a fundamentally unattainable endeavour. The reaction rate coefficients entering the description of cluster growth are often unknown; the knowledge of the geometric configurations of the energetically low-lying structures is often lacking (Bromley et al. 2016. Gobrecht et al. 2017, 2018, Boulangier et al. 2019). The same applies to the description of grain growth and destruction in which case the adsorption and desorption coefficients are often only approximately known since the atomistic details of this process are unknown (Cuppen et al. 2017). In aiming to answer the why question, we have to enter this kinetics playground. Unlike the situation of thermodynamic equilibrium, for which the path is unimportant, an understanding of the path(s) taken toward dust nucleation will be central in our quest. In fact, this addition of specific paths implies an important change in the topology of the landscape. In TE the topology is convergent toward the most stable solutions, the thermodynamic sink. Turned around, this convergent TE solution in the forward direction becomes divergent in the backward direction prohibiting one from going back in time and addressing the why question. Complicating the situation in a non-CE sense implies that we move away from this forward convergent/backward divergent topology, and that we can seek the real roots of mass loss.

\subsection{Dust nucleation: top-down versus bottom-up}

The realistic description of dust formation and growth requires a treatment based on reaction kinetics, which is practically impossible since it would require the solution of a set of order $10^{9}$ rate equations for which most of the reaction rate coefficients are unknown and potentially not even critical. This task is considerably simplified by applying a two-step process in which first suitable seed particles have to form from the gas phase (the nucleation process; see the sidebar titled Nucleation), and in a second step the seed nuclei form the substrate to which molecules are added (Gail \& Sedlmayr 2013). During the last decade, we have witnessed considerable progress in our understanding of the formation of this first seed nuclei using first principles, i.e. following a bottom-up approach. After delving through this section, however, the reader will realise that the situation is still far from satisfactory. 
5.3.1. Some theoretical aspects. Various levels of theory exist for the description of nucleation. One can broadly classify them as the top-down and bottom-up approaches, with some variants in between; a schematized representation is shown in Figure 10. In order to understand the challenges in this field, I have summarized the main principles behind the various approaches in the Supplemental Text. The key points for consideration are summarized here. Before doing so, a general note is appropriate. The term non-equilibrium chemistry, so frequently used in the literature, may convey some information but is devoid of specific information. Only when delving through the detailed mathematical derivations, does it often become clear which physical quantity is not adhering to an equilibrium condition.

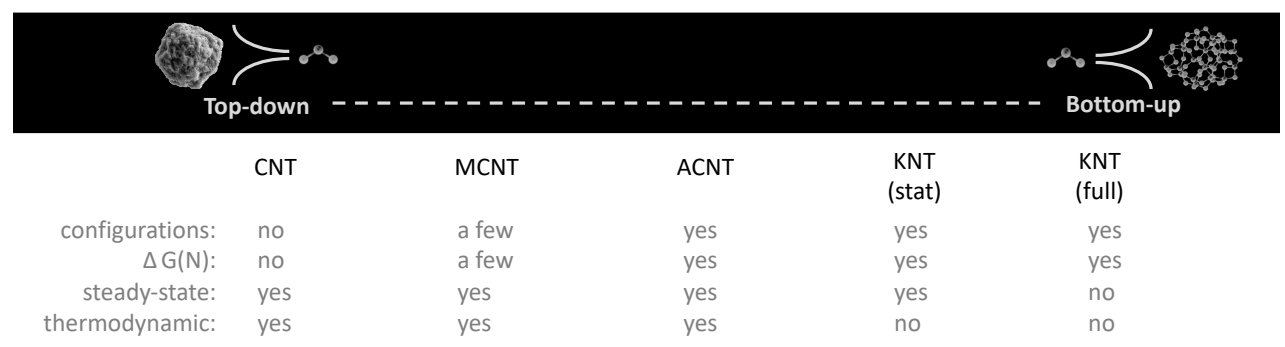

Figure 10

Schematic representation of the different levels of theory used for the calculation of the nucleation rate. The different approaches include the classical nucleation theory (CNT), the modified classical nucleation theory (MCNT), the atomistic classical nucleation theory (ACNT), and the kinetic nucleation theory (KNT). Within KNT, the full time-resolved kinetic model can be solved ('full') or a stationary approach can be pursued $(\mathrm{d}(n, t) / \mathrm{d} t=0$; 'stat'). The different rows indicate if ground-state configurations are calculated using density functional theory (DFT), including the calculation of $\Delta G(N)$, if the nucleation rate is calculated under the assumption of steady-state, and if thermodynamic quantities are needed - in which case 'no' indicates that the nucleation rate can in principle be calculated without recourse to these quantities, although rarely all rate efficiencies are available for this situation to occur.

The challenge of the bottom-up approach is that one needs the geometric configurations with the lowest potential energy (i.e. the global minima) and their respective binding energies for each cluster of size $N$ forming along the pathway toward the macroscopic solid compound. In a first step, one determines a good approximation of the geometric configuration of the molecular clusters by searching the potential energy surfaces for the increasingly complex space of possible arrangements. This first step can already be quite CPU demanding. The candidate configurations are then used as the initial configurations in density functional theory (DFT) calculations to find the lowest energy structures. (See the sidebar titled Density functional theory.) For these structures, the thermodynamic properties (entropy, enthalpy of formation $\Delta_{f} H$, and Gibbs free energy of formation $\Delta_{f} G$ ) are then computed. The Gibbs free energies of formation are then used to calculate $\Delta G(N)$, which is the free energy change associated with the formation of a cluster of size $N$ from the saturated vapour. Using the most modern quantum chemical codes, the DFT method currently allows systems containing some 50 atoms to be calculated (Gobrecht et al. 2018). Owing to the computational challenges, only a few systems of relevance for astrophysical research have been computed at this level of theory, notable examples include $(\mathrm{SiO})_{N}(N=1-20$; Bromley et al. 2016), $\left(\mathrm{TiO}_{2}\right)_{N}(N=1-38$; Jeong et al. 2000, Lee et al. 2015, Lamiel-Garcia et al. 2017. Boulangier et al. 2019), $(\mathrm{C})_{N}\left(N=1-99 ;\right.$ Mauney et al. 2015), $\left(\mathrm{Al}_{2} \mathrm{O}_{3}\right)_{N}$ 


\section{Density functional theory}

To derive the cluster configurations, one needs to solve the Hamiltonian of a system of $N_{e}$ interacting electrons and fixed nuclei for the many-body eigenstate $\Psi_{k}, H\left|\Psi_{k}\right\rangle=E_{k}\left|\Psi_{k}\right\rangle$. Here $H$ denotes the Hamiltonian, $E_{k}$ the energy of the system, and $\Psi_{k}$ the wave function. The basis for density functional theory (DFT) is the Hohenberg-Kohn theorem (Hohenberg \& Kohn 1964), which states that the ground-state energy for an electronic system is a functional of the electron density. Hence, in DFT calculations, the electron density rather than the wave function is used to describe the system. This reduces the dimensions of the system drastically, from $3 N_{e}$ variables to only 3 , the three spatial coordinates. As a result of the Hohenberg-Kohn theorems, the ground-state energy functional $E[n]$ is stationary with respect to the number density $n$. Determination of the ground-state number density $n_{0}(\mathbf{r})$ is then a solution to the constrained variational equation

$$
\frac{\delta}{\delta n(\mathbf{r})}\left[E[n(\mathbf{r})]-\Lambda\left(\int \mathrm{d} \mathbf{r} n(\mathbf{r})-N_{e}\right)\right]=0
$$

with $\mathbf{r}$ the space coordinate and $\Lambda$ the mathematical variable Lagrange multiplier (Kristyan 2013).

$\left(N=1-8 ;\right.$ Li \& Cheng 2012, Gobrecht et al. 2018. Boulangier et al. 2019), and $(\mathrm{MgO})_{N}$ $(N=1-10$; Chen et al. 2014, Boulangier et al. 2019); each of them being proposed as dust precursor candidates in stellar winds.

Given the general lack of quantum chemical data on the cluster properties, various simplifications have been pursued along the path (see Figure 10. In classical nucleation theory (CNT), one uses the capillary approximation to represent the potentially unknown $\Delta G(N)$ via the surface tension of the bulk material, $\sigma_{\infty}$ (see Eq. 62, ). This approximation is only valid for $N \gg 1$ and is also based on the assumption of thermal and chemical equilibrium. It also bypasses the well-known fact that at the nanoscale quantum effects become important, causing the nano-sized particles to behave very differently from their bulk counterpart. Modified classical nucleation theory (MCNT) aims to improve CNT by linking the macroscopic solid bulk and microscopic cluster properties. Using DFT, the cluster properties of a few of the smallest clusters are calculated. The derived $\Delta G(N) / N$ values are then fitted using an analytical formula (see Eq. 73.) to allow extrapolation to larger values of $N$. That way, one accounts for the curvature of the small clusters. For values of $N \rightarrow 1$, the atomistic version (ACNT) is recommended, in which the binding energies as calculated from DFT are explicitly used (Eq. 78).

In the full time-resolved kinetic model, one solves the full time-dependent set of coupled ordinary differential equations describing the rates of change in numbers densities of each cluster of size $N, \mathrm{~d} n(N, t) / \mathrm{d} t$; see Eq. 50 . If the reaction efficiencies are known, the growth and evaporation time scale can be calculated directly, without additional need of the thermodynamic quantities. However, the growth efficiencies are often uncertain and the evaporation efficiencies are mostly unknown. Therefore, methods have been developed based on kinetic nucleation theory (KNT) and thermodynamic arguments (see Supplemental Text). Within KNT, CNT, MCNT, and ACNT it is possible to formulate the stationary nucleation rate, $J_{\star}(t)$, assuming that the cluster densities remain constant over time, i.e. $\mathrm{d} n(N, t) / \mathrm{d} t=0$. Assuming that the growth of clusters only occurs via monomer
Binding energy: energy required to separate constituent atoms/molecules from a system of particles

Gibbs free energy: $\Delta G$, part of the heat of a reaction that can be converted to useful work; if $\Delta G<0$ the reaction is spontaneous, otherwise the reaction is non-spontaneous

Thermodynamic relations: at constant temperature $\Delta G=\Delta H-T \Delta S$, with $H$ the enthalpy, $T$ the temperature and $S$ the entropy of the system 
addition within the context of homogeneous nucleation, $J_{\star}(t)$ can be written as

$$
J_{\star}(t) \simeq z\left(N_{\star}\right) f\left(N_{\star}, t\right) \stackrel{\circ}{n}\left(N_{\star}\right),
$$

where $\stackrel{\circ}{n}(N)$ is the equilibrium density of a cluster of size $N$, and $f(N, t)$ is the monomer attachment rate (see Eq. 60 and Eq. 68). In the framework of CNT, MCNT, and ACNT $z$ is the kinetic Zel'dovich factor which takes a different value for each level of theory (see Eq. 71, Eq. 74 and Eq. 81, but $z=1$ within KNT. $N_{\star}$ is the critical cluster size where $\stackrel{\circ}{n}$ reaches its absolute minimum. The growth reaction of this cluster of size $N_{\star}$ is the bottleneck of the chemical network and hence is the rate limiting step.

For each level of theory, in addition one needs reaction rate coefficients for the calculation of attachment rate $f(N, t)$. One of the most detailed methods (used in astrophysical research) is based on the Rice-Ramsperger-Kassel-Markus (RRKM) theory of chemical activity, employing a solution of the master equation based on the inverse Laplace transform method (Bromley et al. 2016, in the case of $\left.(\mathrm{SiO})_{N}\right)$. In the absence of accurate reaction rates, the rate can be approximated by using the geometrical cross-section and the Maxwell-Boltzmann distribution, so that

$$
k=\pi\left(r_{a}+r_{b}\right)^{2} \sqrt{\frac{8 k T}{\pi \mu}}\left(1+\frac{E_{a}}{k T}\right) \exp \left(-\frac{E_{a}}{k T}\right),
$$

with $r_{a}$ and $r_{b}$ the radii of the colliding species, $\mu$ the reduced mass of the system, and $E_{a}$ the activation energy which is assumed to be zero if unknown (Boulangier et al. 2019). When dealing with large clusters $N \gg 1$, this reaction rate is often denoted as the sticking coefficient $\gamma$ - i.e., the probability that an incoming monomer binds to the target cluster. While in principle it can differ from unity, it is a customary assumption in classical nucleation theory (CNT, MCNT, and ACNT) to assume $\gamma=1$.

5.3.2. Recent theoretical outcomes. Given these various levels of theory, one wonders if taking the bottom-up directive of full kinetic modelling is like taking a sledgehammer to crack a nut. The nucleation process can, mutatis mutandis, be modelled using CNT, the theory which is currently most often used in astrophysical problems. Only a few authors address a comparison between the different approaches (Jeong et al. 2003, Goumans \& Bromley 2012, Mauney et al. 2015, Bromley et al. 2016, Lee et al. 2018). These outcomes are summarized, in a somewhat encyclopedic way, in the Supplemental Text (Section 7.3.5. Before jumping to the bottom line of these comparisons, it is important to realize that each answer given should be interpreted within the context of the particular thermodynamical properties of the medium modelled.

Given the discussion in Section 7.3.5, it seems that resorting to ACNT is sufficient to study dust nucleation in stellar outflows. On the condition that large enough $N$ are reached in the DFT calculations, ACNT yields a fast method to compute the stationary nucleation rate. The only concern then is whether steady state is reached in the pulsationdominated region where dust nucleation is thought to occur. This point has been addressed by Boulangier et al. (2019) who developed a full kinetic model to study the nucleation of $\left(\mathrm{TiO}_{2}\right)_{N},(\mathrm{SiO})_{N},(\mathrm{MgO})_{N}$, and $\left(\mathrm{Al}_{2} \mathrm{O}_{3}\right)_{N}$ in O-rich AGB CSEs. Moreover, they extended the model by including not only monomer interaction, but also polymer interaction. Their results show that cluster growth is time dependent, but for some species the largest clusters are formed in only a few hours, while others take more than $\sim 50$ days - i.e., a significant 
fraction of the AGB/RSG pulsation period during which the thermodynamic properties can change drastically.

Assuming the monomers are present, the full kinetic models of Boulangier et al. (2019) favour $\mathrm{Al}_{2} \mathrm{O}_{3}$ as the primary dust precursor candidate owing to the high temperature at which nucleation can occur $(\sim 1800 \mathrm{~K})$. However, if one starts from an atomic mixture, an acute problem arises because the concentration of the $\mathrm{Al}_{2} \mathrm{O}_{3}$ monomer is almost negligible in the relevant temperature range, and most of the aluminium is in the atomic form. These results suggest that among the possible candidates, $\mathrm{TiO}_{2}$ clusters are the only possible dust precursors. However, the kinetic temperature corresponding to the spatial regions where dust is found is roughly $1500-2000 \mathrm{~K}$ (Norris et al. 2012, Khouri et al. 2016), well above the formation temperature of $\left(\mathrm{TiO}_{2}\right)_{N}=10$ which is around $1000-1200 \mathrm{~K}$. Moreover, a significantly larger fraction of presolar grains of AGB origin contain $\mathrm{Al}_{2} \mathrm{O}_{3}$ rather than $\mathrm{TiO}_{2}$ grains (Choi et al. 1998, Stroud et al. 2004, Bose et al. 2010a), although the expected smaller size of $\mathrm{TiO}_{2}$ grains makes them more difficult to detect. So, alternative routes are now being explored to determine whether larger $\left(\mathrm{Al}_{2} \mathrm{O}_{3}\right)_{n}$ clusters can form bypassing the monomer, and using for example the $\left(\mathrm{Al}_{2} \mathrm{O}_{3}\right)_{2}$ dimer as the main building block, where the dimer is formed from oxidation reactions of the abundant $\mathrm{Al}_{2} \mathrm{O}_{2}$ molecule with $\mathrm{AlO}$, $\mathrm{AlOH}, \mathrm{OH}$, and $\mathrm{H}_{2} \mathrm{O}$ (Gobrecht et al. in prep.). Another possibility might be that large clusters can exist at higher kinetic temperatures than currently suggested by the model calculations, because of thermo-ionic electron emission (Demyk et al. 2004), or possibly radiative cooling (potentially via recurrent fluorescence or vibrational radiative cooling) rather than dissociating. The Nobel-prize winning physicist Richard Feynman famously used as the title of his talk presented to the American Physical Society in Pasadena on December 29, 1959 'There's Plenty of Room at the Bottom'. This statement remains a stellar example of physics prognostication; much of that room has yet to be explored.

\subsection{Discover the path by tracing the route}

So this is where we are. In 2020, we still remain in the realm of the argument formulated by Tielens in 1990, and this despite considerable progress in observations, theoretical models, quantum chemical calculations, and laboratory experiments. The non-CE path followed during dust formation in O-rich environments has still not been identified. The path leading from bottom to up is not merely conceptual — i.e., a gedanken experiment - but rather it is a genuine path that the chemical system adheres to. It is the topology invoked by that path that allows us to reverse the time axis. And actually, we do not need to discover dozens of paths, but one is sufficient since the winner takes it all: if for a given chemical mixture several reaction chains lead to different condensation products, then the reaction chain with the highest nucleation rate wins. Once seeds of one kind start to form, other thermally stable materials will condense on the surface of these seeds, rather than forming seeds themselves. One might question if the quest for this path is too ambitious a target. My answer to this question is 'no'. Thanks to the impressive increase in HPC facilities, it appears we have the beginnings of a solution to this tantalising problem. And although nature is allowed the prerogative of thinking out of the box, even the conventional methods have only been vaguely explored. We have a few indications on the complex quantum chemical route map, but divergent thinking might be needed to identify the correct direction. I use the words 'divergent thinking' because of the huge clumps of dust that are detected in the close vicinity of RSG stars (Cannon et al. 2020): circumstances are far from ideal for dust 
to form under conditions of chromospheric and pulsation activity in a low density region, but far from optimal is clearly not a synonym for impossible. Rather like extremofiles an Earth indicating that life is not vulnerable but persistent, the same seems to hold for dust nucleation.

Not only will quantum chemical calculations help astronomers to discover the path, a new take on understanding circumstellar chemistry was recently launched with the ambitious Stardust project (https://nanocosmos.iff.csic.es/). By building a novel ultra-high vacuum machine combining atomic gas aggregation with in situ characterization techniques, the aim is to reproduce and characterize the bottom-up dust formation process under conditions similar to those of evolved stars. The prime focus of the project is currently on carbon chemistry. By feeding the reactions with individual $\mathrm{C}$ atoms and $\mathrm{H}_{2}$ molecules, it recently has been shown that aromatic species and fullerenes do not form efficiently under these conditions (Martínez et al. 2020). Furthermore, surface reactions can be studied with Stardust. This reveals that reactions on surfaces can process the deposited material and lead to the formation of aromatic systems (in this case benzene, $\mathrm{C}_{6} \mathrm{H}_{6}$, and naphthalene, $\mathrm{C}_{10} \mathrm{H}_{6}$, one of the smallest PAHs), similar to what might be happening in CSEs. However, it should also be noted that the results of Martínez et al. (2020) do not rule out gas-phase pathways for forming aromatic molecules. Although one can conclude from experimental results subject to restricted conditions which reactions are possible, it is logically unsound to conclude which reactions are not possible, particularly over a time span of millions of years within an environment where temperature and density change by orders of magnitude. The phrase by Charles Dickens (1837) in his first novel The Pickwick Papers is particularly apt: 'never say never'.

Moreover, current observational techniques allow one to tackle the problem from an empirical perspective. We are at an important point of history and are now able to spatially resolve the surfaces of stars other than the Sun and to follow the change of the 3D structures through time, and hence gain access to an incredible amount of detail in a 4-dimensional spacetime. Using for example ALMA, we can follow the change in the radial abundance structure of molecules thought to be the building blocks of dust grains ( $\mathrm{TiO}, \mathrm{TiO}_{2}, \mathrm{SiO}$, $\mathrm{AlO}, \mathrm{AlOH}$; Kamiński et al. 2017, Decin et al. 2017, Danilovich et al. 2020) to constrain the fraction of molecules left after dust formation. The challenges here are situated in (1) the lack of collisional rates for the species under study, (2) the need of radiative excitation rates (Einstein A-coefficients) for highly excited states, and (3) the non-spherical and timevarying geometry of the stellar winds (Danilovich et al. 2020). But again, these challenges are within reach thanks to the modern HPC facilities. In collaboration with software engineers, we are on the verge of developing highly efficient algorithms for analysing these data (de Mijolla et al. 2019, De Ceuster et al. 2020ba). The ALMA observatory is not the only one to offer some resolution on this issue. The strongest constraints are obtained from a multi-wavelength multi-instrument setup allowing for contemporaneous observations, a strategy increasingly endorsed by intergovernmental organisations in astronomy. How else would we be able to explain the great dimming of Betelgeuse, the famous red supergiant in the constellation of Orion that is visible with the naked eye and that started fading in December 2019? Not only astronomers, but also the broad public ${ }^{2}$ were wondering if

\footnotetext{
${ }^{2}$ The ESO press-release - https://www.eso.org/public/news/eso2003/ — was by far ESO's most popular press release of 2020 , the study being covered in media outlets with a combined reach of over 2300 million. 
Betelgeuse's dimming meant it was about to explode. Like all red supergiants, Betelgeuse will one day go supernova. One of the currently most favoured working hypothesis for this dimming is the ejection or instant formation of a huge dust clump (Cotton et al. 2020 Safonov et al. 2020, Montargès et al., in prep.); again the seemingly impossible becomes possible, although this might not be exactly what the public has hoped for. The principle by which dust clouds arise turns out to be extraordinarily effective but still obscure. It is clear that a fascinating scientific journey awaits us.

\section{Epilogue}

The mathematical logic of living in a world existing of 4 (classical) spacetime dimensions has guided me through the writing of this review: 1 section was devoted to introducing the reader to the $1 \mathrm{D}$ world of cool ageing stars, 3 sections to three different levels of $3 \mathrm{D}$ complexities; $1+3=4$. These $3 \mathrm{D}$ spatial complexities steer the $4 \mathrm{D}$ path through spacetime of any star.

This discourse brings us naturally to reflections on the next events that might occur on the $4 \mathrm{D}$ path, not only the future of science in this field, but also the future of the stars themselves. A 4-dimensional future in which the time scale of evolution of the 3D complexities cannot be considered as being 'secular' and phenomena are not stationary; $\partial / \partial t \neq 0$. There are two prominent changes which I foresee occurring, the first one within reach of the next few years, the other which might take substantially longer. First, there is the inclusion of binarity in both the forward theoretical and the retrieval modelling of the mass-loss rates of cool ageing stars. This reorientation from a single-star perspective to the inclusion of (sub-)stellar companions will address the how question in a profound way. Second, the exploration of the 3D reality of gas-phase clusters for our understanding of extraterrestrial dust formation is ripe with promise. This is an area within the field of astrochemistry where the why and how question meet each other in an intimate way. For these two advances to occur, both the bottom-up and top-down approach should be pursued in a collaborative effort between astrophysicists, laboratory experimentalists, and quantum chemists. In particular the bottom-up methodology might induce considerable progress in the field given the expected revolution in HPC facilities. I therefore express here the explicit desire for a more intense collaboration with computational experts and HPC facilities. Often we are too addicted to software codes inherited from the past, which are not adapted to current computer performances. That attitude limits the rate of progress in the field, and the rate of improving our understanding of the how and why questions.

This address to the how and why questions is only one small piece in a highly intricate puzzle. And just as you have the medium-sized Golden Russets and the large Haralsons apples, you have why and Why questions. I reserve the capital Why questions for these deeply grounded origin's questions of which the (known) sample size is just one: 'Why did life emerge?' and 'Why did the Universe come about?'. The why question addressed here enters a statistically very different domain with billion of stars exhibiting similar characteristics of 'old and simple', and at the same time of lively and creative as extraterrestrial laboratories. The link between the whys and the Whys can be expressed through the metaphor with the Russian dolls, where each doll gives birth to a smaller doll, logically connected, seemingly separate, but never independent. This brings me to the very first sentences of this review: the Why?, why?, and how? questions driving the curiosity of the human race. 


\section{DISCLOSURE STATEMENT}

The author is not aware of any affiliations, memberships, funding, or financial holdings that might be perceived as affecting the objectivity of this review.

\section{ACKNOWLEDGMENTS}

The author acknowledges the work sessions with the postdoctoral researchers and PhD students during the past few years in Leuven, in particular within the context of the team L.E.E.N. ('Low mass Evolved stars and their ENvironments') meetings, and the various discussions with the colleagues in the ATOMIUM consortium (https://fys.kuleuven.be/ster/research-projects/aerosol/atomium/atomium). The author is grateful for constructive feedback on drafts of the manuscript from Carl Gottlieb, Tom Millar, David Gobrecht, Rens Waters, and the editor Ewine van Dishoeck. C. Gielen is thanked for having produced Figure 3 H.-P. Gail for Figure 4 and J. Bolte and F. De Ceuster for Figure 8. The author received funding from the European Research Council (ERC) under the European Union's Horizon 2020 research and innovation programme (grant agreements No. 646758: AEROSOL with PI L. Decin) and the KU Leuven C1 excellence grant MAESTRO C16/17/007 (PI L. Decin). The author acknowledges the UK Science and Technology Facilities Council (SFTC) IRIS for provision of high-performance computing facilities. This work was partly performed using the Cambridge Service for Data Driven Discovery (CSD3), part of which is operated by the University of Cambridge Research Computing on behalf of the STFC DiRAC HPC Facility. The DiRAC component of CSD3 was funded by BEIS capital funding via STFC capital grants ST/P002307/1 and ST/R002452/1 and STFC operations grant ST/R00689X/1.

\section{LITERATURE CITED}

Adam C, Ohnaka K. 2019. A\&A 628:A132

Agúndez M, Cernicharo J, Guélin M. 2010. ApJ 724:L133-L136

Agúndez M, Cernicharo J, Guélin M. 2014. A\&A 570:A45

Akerman CJ, Carigi L, Nissen PE, Pettini M, Asplund M. 2004. A\&A 414:931-942

Alfvén H. 1942. Nat 150:405-406

Amari S, Anders A, Virag A, Zinner E. 1990. Nat 345:238-240

Arndt TU, Fleischer AJ, Sedlmayr E. 1997. A\&A 327:614-619

Arroyo-Torres B, Wittkowski M, Chiavassa A, Scholz M, Freytag B, et al. 2015. A\&A 575:A50

Auer LH, Woolf NJ. 1965. ApJ 142:182

Balick B, Frank A. 2002. ARA\&A 40:439-486

Baud B, Habing HJ. 1983. A\&A 127:73-83

Beasor ER, Davies B, Smith N, van Loon JT, Gehrz RD, Figer DF. 2020. MNRAS 492:5994-6006

Begemann B, Dorschner J, Henning T, Mutschke H, Gürtler J, et al. 1997. ApJ 476:199-208

Begemann B, Dorschner J, Henning T, Mutschke H, Thamm E. 1994. ApJ 423:L71

Bennett PD. 2010. Chromospheres and Winds of Red Supergiants: An Empirical Look at Outer

Atmospheric Structure, vol. 425 of Astronomical Society of the Pacific Conference Series. 181

Bergeat J, Chevallier L. 2005. A\&A 429:235-246

Bernatowicz TJ, Amari S, Zinner EK, Lewis RS. 1991. ApJ 373:L73

Bertie JE, Labbé HJ, Whalley E. 1969. J. Chem. Phys. 50:4501-4520

Bladh S, Liljegren S, Höfner S, Aringer B, Marigo P. 2019. A\&A 626:A100

Blöcker T. 1995. A\&A 297:727

Boersma C, Hony S, Tielens AGGM. 2006. A\&A 447:213-220 
Bondi H, Hoyle F. 1944. MNRAS 104:273

Bose M, Floss C, Stadermann FJ. 2010a. ApJ 714:1624-1636

Bose M, Floss C, Stadermann FJ, Stroud RM, Speck AK. 2010b. The Origin of Presolar Silica Grains in AGB Stars. In Lunar and Planetary Science Conference, Lunar and Planetary Science Conference

Bose M, Floss C, Stadermann FJ, Stroud RM, Speck AK. 2012. Geochim. Cosmochim. Acta 93:77101

Boulangier J. 2019. Developing a self-consistent AGB wind model. Ph.D. thesis, KU Leuven, Department of Physics and Astronomy

Boulangier J, Gobrecht D, Decin L, de Koter A, Yates J. 2019. MNRAS 489:4890-4911

Bowen GH. 1988. ApJ 329:299

Bromley ST, Gómez Martín JC, Plane JMC. 2016. Physical Chemistry Chemical Physics (Incorporating Faraday Transactions) 18:26913-26922

Bujarrabal V, Alcolea J, Mikołajewska J, Castro-Carrizo A, Ramstedt S. 2018. A\&A 616:L3

Bujarrabal V, Castro-Carrizo A, Alcolea J, Sánchez Contreras C. 2001. A\&A 377:868-897

Bujarrabal V, Castro-Carrizo A, Alcolea J, Santand er-García M, van Winckel H, Sánchez Contreras C. 2016. A\&A 593:A92

Bujarrabal V, Fuente A, Omont A. 1994. A\&A 285:247-271

Buscher DF, Haniff CA, Baldwin JE, Warner PJ. 1990. MNRAS 245:7P

Cameron AGW. 1973. Interstellar Grains in Museums? In Interstellar Dust and Related Topics, eds. JM Greenberg, $\mathrm{HC}$ van de Hulst, vol. 52 of IAU Symposium

Cannon E, Montargès M, de Koter A, Decin L, Min M, et al. 2020. MNRAS subm.

Cernicharo J, Cabezas C, Pardo JR, Agúndez M, Bermúdez C, et al. 2019a. A\&A 630:L2

Cernicharo J, Marcelino N, Agúndez M, Guélin M. 2015. A\&A 575:A91

Cernicharo J, Velilla-Prieto L, Agúndez M, Pardo JR, Fonfría JP, et al. 2019b. A\&A 627:L4

Chen M, Felmy AR, Dixon DA. 2014. Journal of Physical Chemistry A 118:3136-3146

Chen Z, Frank A, Blackman EG, Nordhaus J, Carroll-Nellenback J. 2017. MNRAS 468:4465-4477

Chen Z, Ivanova N, Carroll-Nellenback J. 2020. ApJ 892:110

Cherchneff I. 2006. $A \& A$ 456:1001-1012

Cherchneff I. 2011. $A \& A$ 526:L11

Chiappini C, Romano D, Matteucci F. 2003. MNRAS 339:63-81

Choi BG, Huss GR, Wasserburg GJ, Gallino R. 1998. Science 282:1284

Choi BG, Wasserburg GJ, Huss GR. 1999. ApJ 522:L133-L136

Cohen M, Van Winckel H, Bond HE, Gull TR. 2004. AJ 127:2362-2377

Cotton DV, Bailey J, Horta AD, Norris BRM, Lomax JR. 2020. Research Notes of the American Astronomical Society 4:39

Cox NLJ, Kerschbaum F, van Marle AJ, Decin L, Ladjal D, et al. 2012. A\&A 537:A35

Cuppen HM, Walsh C, Lamberts T, Semenov D, Garrod RT, et al. 2017. Space Sci. Rev. 212:1-58

Curtis HD. 1918. Publications of Lick Observatory 13:9-42

Danilovich T, De Beck E, Black JH, Olofsson H, Justtanont K. 2016. A\&A 588:A119

Danilovich T, Gottlieb CA, Decin L, Richards AMS, Lee KLK, et al. 2020. ApJ subm.

Danilovich T, Teyssier D, Justtanont K, Olofsson H, Cerrigone L, et al. 2015. A\&A 581:A60

Davies B, Beasor ER. 2020a. MNRAS 496:L142-L146

Davies B, Beasor ER. 2020b. MNRAS 493:468-476

Davis AM. 2011. Proceedings of the National Academy of Science 108:19142-19146

De Beck E, Decin L, de Koter A, Justtanont K, Verhoelst T, et al. 2010. A\&A 523:A18

De Beck E, Lombaert R, Agúndez M, Daniel F, Decin L, et al. 2012. A\&A 539:A108

De Ceuster F, Bolte J, Homan W, Maes S, Malfait J, et al. 2020a. MNRAS subm.

De Ceuster F, Homan W, Yates J, Decin L, Boyle P, Hetherington J. 2020b. MNRAS 492:1812-1826

de Jager C, Nieuwenhuijzen H, van der Hucht KA. 1988. A\&AS 72:259-289

de Mijolla D, Viti S, Holdship J, Manolopoulou I, Yates J. 2019. A\&A 630:A117 
de Saint-Exupéry A. 1943. Le Petit Prince. Gallimard

Decin L, Cherchneff I, Hony S, Dehaes S, De Breuck C, Menten KM. 2008. A\&A 480:431-438

Decin L, Cox NLJ, Royer P, Van Marle AJ, Vandenbussche B, et al. 2012. A\&A 548:A113

Decin L, Danilovich T, Gobrecht D, Plane JMC, Richards AMS, et al. 2018a. ApJ 855:113

Decin L, De Beck E, Brünken S, Müller HSP, Menten KM, et al. 2010. A\&A 516:A69

Decin L, Homan W, Danilovich T, de Koter A, Engels D, et al. 2019. Nature Astronomy 3:408-415

Decin L, Hony S, de Koter A, Justtanont K, Tielens AGGM, Waters LBFM. 2006. A\&A 456:549-563

Decin L, Montargès M, Richards AMS, Gottlieb CA, Homan W, et al. 2020. Science in press

Decin L, Richards AMS, Danilovich T, Homan W, Nuth JA. 2018b. A\&A 615:A28

Decin L, Richards AMS, Millar TJ, Baudry A, De Beck E, et al. 2016. A\&A 592:A76

Decin L, Richards AMS, Neufeld D, Steffen W, Melnick G, Lombaert R. 2015. A\&A 574:A5

Decin L, Richards AMS, Waters LBFM, Danilovich T, Gobrecht D, et al. 2017. A\&A 608:A55

Decin L, Royer P, Cox NLJ, Vandenbussche B, Ottensamer R, et al. 2011. A\&A 534:A1

Demyk K, van Heijnsbergen D, von Helden G, Meijer G. 2004. A\&A 420:547-552

Deutsch AJ. 1956. ApJ 123:210

Dickens C. 1837. The Posthumous Papers of the Pickwick Club, Containing a Faithful Record of the Perambulations, Perils, Travels, Adventures and Sporting Transactions of the Corresponding Members. Chapman \& Hall

Dominik C, Sedlmayr E, Gail HP. 1993. A\&A 277:578

Dorschner J, Begemann B, Henning T, Jaeger C, Mutschke H. 1995. A\& A 300:503

Dyck HM, Benson JA, Ridgway ST, Dixon DJ. 1992. AJ 104:1982

Eggleton PP. 1983. ApJ 268:368-369

Einstein A. 1905. Annalen der Physik 323:639-641

Einstein A. 1915. Sitzungsberichte der Königlich Preußischen Akademie der Wissenschaften (Berlin :844-847

Ekström S, Georgy C, Eggenberger P, Meynet G, Mowlavi N, et al. 2012. A\&A 537:A146

El Mellah I, Bolte J, Decin L, Homan W, Keppens R. 2020. A\&A in press

Eriksson K, Nowotny W, Höfner S, Aringer B, Wachter A. 2014. A\&A 566:A95

Feast MW. 1992. Pulsation and instability amongst the most luminous stars. In Instabilities in Evolved Super- and Hypergiants, eds. C de Jager, H Nieuwenhuijzen

Feynman RP. 1959. There's Plenty of Room at the Bottom

Floss C, Stadermann FJ, Bose M. 2008. ApJ 672:1266-1271

Flower DR, Pineau des Forêts G. 2015. A\&A 578:A63

Ford KES, Neufeld DA, Goldsmith PF, Melnick GJ. 2003. ApJ 589:430-438

Ford KES, Neufeld DA, Schilke P, Melnick GJ. 2004. ApJ 614:990-1006

Fox MW, Wood PR. 1982. ApJ 259:198-212

Freytag B, Höfner S, Liljegren S. 2019. 3D modelling of AGB stars with CO5BOLD. In IAU Symposium, eds. F Kerschbaum, M Groenewegen, H Olofsson, vol. 343 of IAU Symposium

Freytag B, Liljegren S, Höfner S. 2017. A\&A 600:A137

Freytag B, Steffen M, Dorch B. 2002. Astronomische Nachrichten 323:213-219

Fulton B. 2019. Frequency of Gaseous Planets Beyond the Ice Line. Paper presented at the meeting of Extreme Solar Systems IV, August 19-23, 2019, Reykjavik, Iceland

Fulton BJ, Petigura EA. 2018. AJ 156:264

Gail HP, Sedlmayr E. 1999. A\&A 347:594-616

Gail HP, Sedlmayr E. 2013. Physics and Chemistry of Circumstellar Dust Shells

García-Segura G, Langer N, Różyczka M, Franco J. 1999. ApJ 517:767-781

Gauger A, Gail HP, Sedlmayr E. 1990. A\&A 235:345-361

Gehrz RD, Woolf NJ. 1971. ApJ 165:285

Gillett FC, Low FJ, Stein WA. 1968. ApJ 154:677

Gilliland RL, Dupree AK. 1996. ApJ 463:L29

Gilman RC. 1969. ApJ 155:L185 
Gilra DP. 1973. Dust Particles and Molecules in the Extended Atmospheres of Carbon Stars. In Interstellar Dust and Related Topics, eds. JM Greenberg, HC van de Hulst, vol. 52 of IAU Symposium

Glaccum W. 1995. Infrared dust features of late-type stars and planetary nebulae., vol. 73 of Astronomical Society of the Pacific Conference Series. 395-396

Glass IS, Evans TL. 1981. Nat 291:303-304

Glassgold AE. 1999. Circumstellar Chemistry of AGB Winds. In Asymptotic Giant Branch Stars, eds. T Le Bertre, A Lebre, C Waelkens, vol. 191 of IAU Symposium

Gobrecht D, Cherchneff I, Sarangi A, Plane JMC, Bromley ST. 2016. A\&A 585:A6

Gobrecht D, Cristallo S, Piersanti L, Bromley ST. 2017. ApJ 840:117

Gobrecht D, Decin L, Cristallo S, Bromley ST. 2018. Chemical Physics Letters 711:138-147

Godard B, Pineau des Forêts G, Lesaffre P, Lehmann A, Gusdorf A, Falgarone E. 2019. A\&A 622:A100

Goderis S, Chakrabarti R, Debaille V, Kodolányi J. 2016. Jour. Anal. At. Spectrom. 31:841

Goebel JH, Moseley SH. 1985. ApJ 290:L35-L39

Goldman SR, van Loon JT, Zijlstra AA, Green JA, Wood PR, et al. 2017. MNRAS 465:403-433

Goldreich P, Scoville N. 1976. ApJ 205:144-154

Gottlieb CA, Decin L, Richards AMS, Wallström SHJ, Millar TJ, et al. 2020. A\&A subm.

Goumans TPM, Bromley ST. 2012. MNRAS 420:3344-3349

Groenewegen MAT. 2014. A\&A 561:L11

Groenewegen MAT, Marigo P. 2004. Synthetic AGB Evolution. 105-148

Groenewegen MAT, Sloan GC, Soszyński I, Petersen EA. 2009. A\&A 506:1277-1296

Groenewegen MAT, Vlemmings WHT, Marigo P, Sloan GC, Decin L, et al. 2016. A\&A 596:A50

Groh JH, Meynet G, Georgy C, Ekström S. 2013. A\&A 558:A131

Grossman L, Larimer JW. 1974. Reviews of Geophysics and Space Physics 12:71-101

Guélin M, Patel NA, Bremer M, Cernicharo J, Castro-Carrizo A, et al. 2018. A\&A 610:A4

Guerrero MA, Chu YH, Manchado A, Kwitter KB. 2003. AJ 125:3213-3221

Gustafsson B, Höfner S. 2003. Atmospheres of AGB Stars. In Asymptotic giant branch stars

Haniff CA, Buscher DF. 1998. A\&A 334:L5-L8

Henning T. 2010. ARA\&A 48:21-46

Henning T, Begemann B, Mutschke H, Dorschner J. 1995. A\&AS 112:143

Henning T, Mutschke H. 2001. Spectrochimica Acta 57:815-824

Henning T, Stognienko R. 1996. A\&A 311:291-303

Heras AM, Hony S. 2005. A\&A 439:171-182

Hertzsprung E. 1905. Zeitschrift Fur Wissenschaftliche Photographie 3:442-449

Hertzsprung E. 1911. Publikationen des Astrophysikalischen Observatoriums zu Potsdam 22:A1A 40.1

Hinkle KH, Aringer B, Lebzelter T, Martin CL, Ridgway ST. 2000. A\&A 363:1065-1080

Höfner S. 2008. A\&A 491:L1-L4

Höfner S, Andersen AC. 2007. A\&A 465:L39-L42

Höfner S, Bladh S, Aringer B, Ahuja R. 2016. A\&A 594:A108

Höfner S, Feuchtinger MU, Dorfi EA. 1995. A\&A 297:815

Höfner S, Freytag B. 2019. A\&A 623:A158

Höfner S, Olofsson H. 2018. A\&AR 26:1

Hohenberg P, Kohn W. 1964. Physical Review 136:864-871

Homan W, Danilovich T, Decin L, de Koter A, Nuth J, Van de Sande M. 2018a. A\&A 614:A113

Homan W, Richards A, Decin L, de Koter A, Kervella P. 2018b. A\&A 616:A34

Hony S, Heras AM, Molster FJ, Smolders K. 2009. A\&A 501:609-617

Hoyle F, Lyttleton RA. 1939. Proceedings of the Cambridge Philosophical Society 35:405

Hoyle F, Wickramasinghe NC. 1962. MNRAS 124:417

Iben I. J. 1984. ApJ 277:333-354 
Ivanova N, Justham S, Chen X, De Marco O, Fryer CL, et al. 2013. A\&AR 21:59

Jaeger C, Molster FJ, Dorschner J, Henning T, Mutschke H, Waters LBFM. 1998. A\&A 339:904-916

Jaeger C, Mutschke H, Begemann B, Dorschner J, Henning T. 1994. A\& A 292:641-655

Jäger C, Il'in VB, Henning T, Mutschke H, Fabian D, et al. 2003. J. Quant. Spectrosc. Radiat. Transfer 79-80:765

Jeffers SV, Min M, Waters LBFM, Canovas H, Pols OR, et al. 2014. A\&A 572:A3

Jeong KS, Chang C, Sedlmayr E, Sülzle D. 2000. Journal of Physics B Atomic Molecular Physics 33:3417-3430

Jeong KS, Winters JM, Le Bertre T, Sedlmayr E. 2003. A\&A 407:191-206

Josselin E, Plez B. 2007. A\&A 469:671-680

Justtanont K. 1992. Mass loss from evolved late type stars. Ph.D. thesis, University of London, University College London (United Kingdom)

Justtanont K, Feuchtgruber H, de Jong T, Cami J, Waters LBFM, et al. 1998. A\&A 330:L17-L20

Kamiński T. 2019. A\&A 627:A114

Kamiński T, Müller HSP, Schmidt MR, Cherchneff I, Wong KT, et al. 2017. A\&A 599:A59

Karovicova I, Wittkowski M, Ohnaka K, Boboltz DA, Fossat E, Scholz M. 2013. A\&A 560:A75

Karovska M, Nisenson P, Beletic J. 1993. ApJ 402:311

Kee N, Sundqvist J, Decin L, de Koter A, Sana H. 2020. A\&A subm.

Kemper F, de Koter A, Waters LBFM, Bouwman J, Tielens AGGM. 2002. A\&A 384:585-593

Kervella P, Gallenne A, Remage Evans N, Szabados L, Arenou F, et al. 2019. A\&A 623:A116

Kervella P, Homan W, Richards AMS, Decin L, McDonald I, et al. 2016. A\&A 596:A92

Khouri T, Maercker M, Waters LBFM, Vlemmings WHT, Kervella P, et al. 2016. A\&A 591:A70

Khouri T, Waters LBFM, de Koter A, Decin L, Min M, et al. 2015. A\&A 577:A114

Kim H, Liu SY, Hirano N, Zhao-Geisler R, Trejo A, et al. 2015. ApJ 814:61

Kim H, Liu SY, Taam RE. 2019. ApJS 243:35

Kim H, Taam RE. 2012. ApJ 759:59

Kitamura R, Pilon L, Jonasz M. 2007. Appl. Opt. 46:8118-8133

Kochanek CS, Khan R, Dai X. 2012. ApJ 759:20

Koike C, Kaito C, Yamamoto T, Shibai H, Kimura S, Suto H. 1995. Icarus 114:203-214

Koike C, Shibai H, Tuchiyama A. 1993. MNRAS 264:654

Koike C, Tsuchiyama A, Shibai H, Suto H, Tanabé T, et al. 2000. A\&A 363:1115-1122

Kristyan S. 2013. Journal of Theoretical and Applied Physics 7:61

Kudritzki RP, Reimers D. 1978. A\&A 70:227-239

Lagadec E, Zijlstra AA. 2008. MNRAS 390:L59-L63

Lamers HJGLM, Cassinelli JP. 1999. Introduction to Stellar Winds

Lamiel-Garcia O, Cuko A, Calatayud M, Illas F, Bromley ST. 2017. Nanoscale 9:1049

Lee G, Helling C, Giles H, Bromley ST. 2015. A\&A 575:A11

Lee GKH, Blecic J, Helling C. 2018. A\&A 614:A126

Lesaffre P, Pineau des Forêts G, Godard B, Guillard P, Boulanger F, Falgarone E. 2013. A\&A 550:A106

Lewis RS, Ming T, Wacker JF, Anders E, Steel E. 1987. Nat 326:160-162

Li R, Cheng L. 2012. Comput. Theor. Chem 996:125

Li X, Millar TJ, Walsh C, Heays AN, van Dishoeck EF. 2014. A\&A 568:A111

Liljegren S, Höfner S, Freytag B, Bladh S. 2018. A\&A 619:A47

Liu ZW, Stancliffe RJ, Abate C, Matrozis E. 2017. ApJ 846:117

Lodders K, Amari S. 2005. Chemie der Erde / Geochemistry 65:93-166

Lombaert R, Decin L, Royer P, de Koter A, Cox NLJ, et al. 2016. A\&A 588:A124

Maercker M, Mohamed S, Vlemmings WHT, Ramstedt S, Groenewegen MAT, et al. 2012. Nat 490:232-234

Martin PG, Rogers C. 1987. ApJ 322:374

Martínez L, Santoro G, Merino P, Accolla M, Lauwaet K, et al. 2020. Nature Astronomy 4:97-105 
Mastrodemos N, Morris M. 1999. ApJ 523:357-380

Matsuura M, Sloan GC, Zijlstra AA, Wood PR, Harris JG, et al. 2007. Infrared Molecular Bands of Carbon-Rich Stars in Nearby Galaxies. In Why Galaxies Care About AGB Stars: Their Importance as Actors and Probes, eds. F Kerschbaum, C Charbonnel, RF Wing, vol. 378 of Astronomical Society of the Pacific Conference Series

Mauney C, Buongiorno Nardelli M, Lazzati D. 2015. ApJ 800:30

Mauron N, Huggins PJ. 1999. A\&A 349:203-208

Mauron N, Huggins PJ. 2006. A\&A 452:257-268

Maury AC, Pickering EC. 1897. Annals of Harvard College Observatory 28:1-128

McDonald I, Trabucchi M. 2019. MNRAS 484:4678-4682

McDonald I, Zijlstra AA. 2015. MNRAS 448:502-521

McDonald I, Zijlstra AA. 2016. ApJ 823:L38

McGuire BA. 2018. ApJS 239:17

Melnick GJ, Neufeld DA, Ford KES, Hollenbach DJ, Ashby MLN. 2001. Nat 412:160-163

Menut JL, Gendron E, Schartmann M, Tuthill P, Lopez B, et al. 2007. MNRAS 376:L6-L10

Mihalas D, Hummer DG. 1974. ApJS 28:343-372

Miszalski B, Acker A, Moffat AFJ, Parker QA, Udalski A. 2009. A\&A 496:813-825

Moe M, Di Stefano R. 2017. ApJS 230:15

Molster FJ, Waters LBFM, Tielens AGGM, Barlow MJ. 2002a. A\&A 382:184-221

Molster FJ, Waters LBFM, Tielens AGGM, Barlow MJ. 2002b. A\&A 382:184-221

Molster FJ, Waters LBFM, Trams NR, Van Winckel H, Decin L, et al. 1999a. A\&A 350:163-180

Molster FJ, Yamamura I, Waters LBFM, Tielens AGGM, de Graauw T, et al. 1999b. Nat 401:563565

Montargès M, Homan W, Keller D, Clementel N, Shetye S, et al. 2019. MNRAS 485:2417-2430

Mutschke H, Begemann B, Dorschner J, Guertler J, Gustafson B, et al. 1998. A\&A 333:188-198

Mutschke H, Posch T, Fabian D, Dorschner J. 2002. A\&A 392:1047-1052

Neri R, Kahane C, Lucas R, Bujarrabal V, Loup C. 1998. A\&AS 130:1-64

Neugent KF, Levesque EM, Massey P, Morrell NI. 2019. ApJ 875:124

Nielsen EL, De Rosa RJ, Macintosh B, Wang JJ, Ruffio JB, et al. 2019. AJ 158:13

Nieuwenhuijzen H, de Jager C. 1990. A\&A 231:134-136

Nittler LR, Alexander CMO, Gallino R, Hoppe P, Nguyen AN, et al. 2008. ApJ 682:1450-1478

Nittler LR, Ciesla F. 2016. ARA\&A 54:53-93

Norris BR, Tuthill PG, Ireland MJ, Lacour S, Zijlstra AA, et al. 2012. Nat 484:220-222

O'Dell CR, Balick B, Hajian AR, Henney WJ, Burkert A. 2002. AJ 123:3329-3347

O'Dell CR, McCullough PR, Meixner M. 2004. AJ 128:2339-2356

O'Gorman E, Kervella P, Harper GM, Richards AMS, Decin L, et al. 2017. A\&A 602:L10

Ohnaka K. 2014. A\&A 568:A17

Ohnaka K, Driebe T, Hofmann KH, Leinert C, Morel S, et al. 2006. A\&A 445:1015-1029

Ohnaka K, Weigelt G, Hofmann KH. 2017a. A\&A 597:A20

Ohnaka K, Weigelt G, Hofmann KH. 2017b. Nat 548:310-312

Olofsson H. 2005. Molecular Abundances in AGB Circumstellar Envelopes. In Astrochemistry: Recent Successes and Current Challenges, eds. DC Lis, GA Blake, E Herbst, vol. 231 of IAU Symposium

Onaka T, de Jong T, Willems FJ. 1989. A\&A 218:169-179

Paczyński B. 1970. Acta Astronomica 20:47

Paczyński B. 1971. ARA\&A 9:183

Paladini C, Baron F, Jorissen A, Le Bouquin JB, Freytag B, et al. 2018. Nat 553:310-312

Parker EN. 1958. ApJ 128:664

Parker EN. 1960. ApJ 132:821

Parker QA, Acker A, Frew DJ, Hartley M, Peyaud AEJ, et al. 2006. MNRAS 373:79-94

Patzer A. 1998. Non-equilibrium effects on chemistry and dust formation in circumstellar outflows. 
Ph.D. thesis, Technical University of Berlin

Patzer ABC, Gauger A, Sedlmayr E. 1998. A\&A 337:847-858

Pitman KM, Hofmeister AM, Corman AB, Speck AK. 2008. A\&A 483:661-672

Podsiadlowski P, Mohamed S. 2007. Baltic Astronomy 16:26-33

Posch T, Kerschbaum F, Fabian D, Mutschke H, Dorschner J, et al. 2003. ApJS 149:437-445

Posch T, Kerschbaum F, Mutschke H, Dorschner J, Jäger C. 2002. A\&A 393:L7-L10

Posch T, Kerschbaum F, Mutschke H, Fabian D, Dorschner J, Hron J. 1999. A\& A 352:609-618

Preibisch T, Ossenkopf V, Yorke HW, Henning T. 1993. A\&A 279:577-588

Ramstedt S, Maercker M, Olofsson G, Olofsson H, Schöier FL. 2011. A\&A 531:A148

Ramstedt S, Mohamed S, Olander T, Vlemmings WHT, Khouri T, Liljegren S. 2018. A\&A 616:A61

Ramstedt S, Mohamed S, Vlemmings WHT, Maercker M, Montez R, et al. 2014. A\&A 570:L14

Ramstedt S, Schöier FL, Olofsson H, Lundgren AA. 2008. A\&A 487:645-657

Randall SK, Trejo A, Humphreys EML, Kim H, Wittkowski M, et al. 2020. A\&A 636:A123

Reid N, Tinney C, Mould J. 1990. ApJ 348:98

Reimers D. 1975. Memoires of the Societe Royale des Sciences de Liege 8:369-382

Renzini A. 1981. Red giants as precursors of planetary nebulae, vol. 88 of Astrophysics and Space Science Library. 431-446

Renzini A, Fusi Pecci F. 1988. ARA\&A 26:199-244

Ryde N, Eriksson K, Gustafsson B, Lindqvist M, Olofsson H. 1998. Ap\&SS 255:301-302

Sabach E, Soker N. 2018. MNRAS 479:2249-2255

Safonov B, Dodin A, Burlak M, Goliguzova M, Fedoteva A, et al. 2020. arXiv e-prints :arXiv:2005.05215

Sahai R, Morris MR, Villar GG. 2011. AJ 141:134

Saladino MI, Pols OR. 2019. A\&A 629:A103

Saladino MI, Pols OR, van der Helm E, Pelupessy I, Portegies Zwart S. 2018. A\&A 618:A50

Salasnich B, Bressan A, Chiosi C. 1999. A\&A 342:131-152

Sana H, de Mink SE, de Koter A, Langer N, Evans CJ, et al. 2012. Science 337:444

Schöier FL, Olofsson H, Lundgren AA. 2006. A\& A 454:247-255

Schöier FL, Ramstedt S, Olofsson H, Lindqvist M, Bieging JH, Marvel KB. 2013. A\&A 550:A78

Schöier FL, Ryde N, Olofsson H. 2002. A\&A 391:577-586

Schrettle F, Kant C, Lunkenheimer P, Mayr F, Deisenhofer J, Loidl A. 2012. Eur. Phys. Jour. B 85:164

Schröder KP, Smith RC. 2008. MNRAS 386:155-163

Schwarzschild M. 1975. ApJ 195:137-144

Scicluna P, Siebenmorgen R, Wesson R, Blommaert JADL, Kasper M, et al. 2015. A\&A 584:L10

Shawl SJ. 1972. Observations and Models of Polarization in Late - Stars. Ph.D. thesis, THE UNIVERSITY OF TEXAS AT AUSTIN.

Simis YJW, Icke V, Dominik C. 2001. A\&A 371:205-221

Smartt SJ, Eldridge JJ, Crockett RM, Maund JR. 2009. MNRAS 395:1409-1437

Smolders K, Acke B, Verhoelst T, Blommaert JADL, Decin L, et al. 2010. A\&A 514:L1

Soker N. 1998. ApJ 496:833-841

Soker N. 2001. ApJ 558:157-164

Soker N, Harpaz A. 1992. PASP 104:923

Solomon P, Jefferts KB, Penzias AA, Wilson RW. 1971. ApJ 163:L53

Srinivasan S, Meixner M, Leitherer C, Vijh U, Volk K, et al. 2009. AJ 137:4810-4823

Straniero O, Chieffi A, Limongi M, Busso M, Gallino R, Arlandini C. 1997. ApJ 478:332-339

Stroud RM, Nittler LR, Alexand er CMO. 2004. Science 305:1455-1457

Su KYL, Chu YH, Rieke GH, Huggins PJ, Gruendl R, et al. 2007. ApJ 657:L41-L45

Takigawa A, Stroud RM, Nittler LR, Alexand er CMO. 2014. A Titanium Oxide Grain Within a Presolar Corundum. In 77th Annual Meeting of the Meteoritical Society, vol. 77

Tielens AGGM. 1990. Towards a Circumstellar Silicate Mineralogy. In From Miras to Planetary 
Nebulae: Which Path for Stellar Evolution?, eds. MO Mennessier, A Omont

Tielens AGGM. 2005. The Physics and Chemistry of the Interstellar Medium

Tielens AGGM, Waters LBFM, Molster FJ, Justtanont K. 1998. Ap\&SS 255:415-426

Tikhonov VV, Boyarskii DA, Polyakova ON. 2018. Advances in Space Research 62:2692-2700

Trabucchi M, Wood PR, Montalbán J, Marigo P, Pastorelli G, Girardi L. 2017. ApJ 847:139

Trabucchi M, Wood PR, Montalbán J, Marigo P, Pastorelli G, Girardi L. 2019. MNRAS 482:929-

949

Tropf WJ, Thomas ME. 1991. Aluminum Oxynitride (ALON) Spinel. In Handbook of Optical Constants of Solids II, ed. ED Palik

Ueta T, Murakawa K, Meixner M. 2007. AJ 133:1345-1360

Van de Sande M, Decin L, Lombaert R, Khouri T, de Koter A, et al. 2018a. A\&A 609:A63

Van de Sande M, Millar TJ. 2019. ApJ 873:36

Van de Sande M, Sundqvist JO, Millar TJ, Keller D, Homan W, et al. 2018b. A\&A 616:A106

van Loon JT, Cioni MRL, Zijlstra AA, Loup C. 2005. A\&A 438:273-289

van Marle AJ, Meliani Z, Keppens R, Decin L. 2011. ApJ 734:L26

Vassiliadis E, Wood PR. 1993. ApJ 413:641

Velilla Prieto L, Cernicharo J, Quintana-Lacaci G, Agúndez M, Castro-Carrizo A, et al. 2015. ApJ 805:L13

Verhoelst T, van der Zypen N, Hony S, Decin L, Cami J, Eriksson K. 2009. A\&A 498:127-138

Wachter A, Schröder KP, Winters JM, Arndt TU, Sedlmayr E. 2002. A\&A 384:452-459

Wagenhuber J, Groenewegen MAT. 1998. A\&A 340:183-195

Walmswell JJ, Eldridge JJ. 2012. MNRAS 419:2054-2062

Warren SG. 1984. Appl. Opt. 23:1206-1225

Waters LBFM, Molster FJ, de Jong T, Beintema DA, Waelkens C, et al. 1996. A\&A 315:L361-L364

Waters LBFM, Molster FJ, Hony S, Kemper F, Yamamura I, et al. 2000. ISO Spectroscopy of Circumstellar Dust. In Thermal Emission Spectroscopy and Analysis of Dust, Disks, and Regoliths, eds. ML Sitko, AL Sprague, DK Lynch, vol. 196 of Astronomical Society of the Pacific Conference Series

Weaver H, Williams DRW, Dieter NH, Lum WT. 1965. Nat 208:29-31

Weigelt G, Balega Y, Bloecker T, Fleischer AJ, Osterbart R, Winters JM. 1998. A\&A 333:L51-L54

Weinberg S. 1994. Dreams of a Final Theory. Vintage: New York

Weymann R. 1962a. ApJ 136:476

Weymann R. 1962b. ApJ 136:844

Wickramasinghe NC, Donn BD, Stecher TP. 1966. ApJ 146:590

Wiegert J, Groenewegen MAT, Jorissen A, Decin L, Danilovich T. 2020. A\&A 642:A142

Wildt R. 1933. Zeitschrift für Astrophysik 6:345

Willacy K, Cherchneff I. 1998. A\&A 330:676-684

Willacy K, Millar TJ. 1997. A\&A 324:237-248

Willson LA. 2000. ARA\&A 38:573-611

Wilson WJ, Barrett AH. 1968. Science 161:778-779

Wittgenstein L. 1921. Tractatus Logico-Philosophicus. Annalen der Naturphilosphie

Wittkowski M, Hofmann KH, Höfner S, Le Bouquin JB, Nowotny W, et al. 2017. A\&A 601:A3

Woitke P. 2006a. A\&A 452:537-549

Woitke P. 2006b. A\&A 460:L9-L12

Wong KT, Kamiński T, Menten KM, Wyrowski F. 2016. A\&A 590:A127

Wood PR. 1990. Mira variables: pulsation, mass loss and evolution. In Confrontation Between Stellar Pulsation and Evolution, eds. C Cacciari, G Clementini, vol. 11 of Astronomical Society of the Pacific Conference Series

Wood PR. 2015. MNRAS 448:3829-3843

Wood PR, Alcock C, Allsman RA, Alves D, Axelrod TS, et al. 1999. MACHO observations of LMC red giants: Mira and semi-regular pulsators, and contact and semi-detached binaries. 
In Asymptotic Giant Branch Stars, eds. T Le Bertre, A Lebre, C Waelkens, vol. 191 of IAU Symposium

Woolf NJ, Ney EP. 1969. ApJ 155:L181

Woosley SE, Heger A. 2012. ApJ 752:32

Yoon SC, Cantiello M. 2010. ApJ 717:L62-L65

Zhang B, Reid MJ, Menten KM, Zheng XW. 2012. ApJ 744:23

Zijlstra AA, Lagadec E, Sloan G, Matsuura M. 2009. The AGB Superwind in Nearby Galaxies. In The Biggest, Baddest, Coolest Stars, eds. DG Luttermoser, BJ Smith, RE Stencel, vol. 412 of Astronomical Society of the Pacific Conference Series 


\section{SUPPLEMENTAL ONLINE MATERIAL}

\subsection{Mass-loss rate prescriptions}

The first empirical mass-loss rate prescription for cool ageing stars was derived in 1975 by Reimers (1975)

$$
\dot{M}_{\mathrm{R}}=4 \times 10^{-13} \eta L / g R,
$$

with $\dot{M}$ the mass-loss rate in units of $\mathrm{M}_{\odot} \mathrm{yr}^{-1}, \eta$ a unitless parameter of the order of unity, and the stellar luminosity $L$, gravity $g$, and radius $R$ in solar units; see also Eq. 11 This relation was derived for RSG stars, but later was often applied to AGB stars by adapting the $\eta$-parameter. Kudritzki \& Reimers (1978) calibrated the $\eta$-parameter for RSG stars and arrived at $\eta \simeq 1.375$; Renzini \& Fusi Pecci (1988) derived $\eta \simeq 0.4$ for AGB stars. Since then, various other empirical, semi-empirical, and theoretical mass-loss rate prescriptions have been derived, such as the ones mentioned in Section 2.2.3 In this section, I review in chronological order some of these parametric relations often used in the AGB and RSG community, in particular for stellar evolution and population synthesis modelling. With the aim of comparing various mass-loss prescriptions for their explicit dependence on the fundamental parameters $\mathrm{L}_{\star}$ and $\mathrm{T}_{\text {eff }}$, Reimers' relation is rewritten as

$$
\log \dot{M}_{\mathrm{R}}=-4.876+\log \eta+1.5 \log \mathrm{L}_{\star}-2 \log \mathrm{T}_{\text {eff }}-2 \log \mathrm{M}_{\star},
$$

where $\log$ refers to the base-10 logarithm, and $\mathrm{M}_{\star}$ is in solar units.

de Jager et al. (1988) collected mass-loss rates determined for 271 Galactic stars of spectral types $\mathrm{O}$ through $\mathrm{M}$. The mass-loss rates could be reproduced by a single empirical formula dependent on $\mathrm{T}_{\text {eff }}$ and $\mathrm{L}_{\star}$

$$
\log \dot{M}_{\mathrm{J} 88}=-8.158+1.769 \log \mathrm{L}_{\star}-1.676 \log \mathrm{T}_{\mathrm{eff}} .
$$

The original de Jager formulation did not account for the stellar mass. An improved relation was therefore proposed by Nieuwenhuijzen \& de Jager (1990)

$$
\log \dot{M}_{\mathrm{NJ} 90}=-7.93+1.64 \log \mathrm{L}_{\star}-1.61 \log \mathrm{T}_{\text {eff }}+0.16 \log \mathrm{M}_{\star},
$$

but it can be seen that the dependence on the stellar mass is weak.

Vassiliadis \& Wood (1993) combined an empirical relation between the mass-loss rate and the pulsation period ( $P$, in days) for Mira-type variables

$$
\begin{array}{lll}
\log \dot{M}_{\mathrm{VW} 93}=-11.4+0.0123 P & \left(\text { for } \mathrm{M}_{\star} \leq 2.5 \mathrm{M}_{\odot}\right) & 27 . \\
\log \dot{M}_{\mathrm{VW} 93}=-11.4+0.0125\left[P-100\left(\mathrm{M}_{\star}-2.5\right)\right] & \left(\text { for } \mathrm{M}_{\star}>2.5 \mathrm{M}_{\odot}\right) & 28 .
\end{array}
$$

with a theoretical period-mass-radius relation (or period-mass-luminosity-effective temperature relation)

$$
\log P=12.52+0.97 \log \mathrm{L}_{\star}-3.88 \log \mathrm{T}_{\mathrm{eff}}-0.9 \log \mathrm{M}_{\star}
$$

to obtain a semi-empirical mass-loss rate formula for stars with periods between $\sim 300$ 800 days. For larger periods, the mass-loss rate is given by the single-scattering radiation pressure limit $\dot{M}=\mathrm{L}_{\star} / c v_{e}$, where the expansion velocity, $v_{e}$ (in $\mathrm{km} \mathrm{s}^{-1}$ ), was calculated using

$$
v_{e}=-13.5+0.056 P \text {. }
$$


Blöcker (1995) used a modification of the Reimers' law for his theoretical AGB evolutionary calculations, by multiplying the Reimers' rate by $L^{2.7}$ and adding a dependence on the mass at the zero-age-main-sequence (ZAMS) as $M_{\mathrm{ZAMS}}^{-2.1}$. The modifications were guided by theoretical mass-loss rate calculations for fundamental mode Mira-type pulsators for which the period-mass-radius relation was taken as

$$
\log P=-1.92+0.93 \log \mathrm{L}_{\star}-3.72 \log \mathrm{T}_{\mathrm{eff}}-0.73 \log \mathrm{M}_{\star} .
$$

By setting, somewhat arbitrarily, $M_{\text {ZAMS }}$ to the actual total mass, the following theoretical relation was obtained

$$
\log \dot{M}_{\mathrm{B} 95}=-13.19+\log \eta+3.7 \log \mathrm{L}_{\star}-2 \log \mathrm{T}_{\mathrm{eff}}-3.1 \log \mathrm{M}_{\star} .
$$

Using the period-luminosity relation as derived by Feast (1992) for 15 RSG stars in the Large Magellanic Cloud (LMC) and mass-loss rates derived by Reid et al. (1990), Salasnich et al. (1999) derived an empirical relation

$$
\log \dot{M}_{\mathrm{S} 99}=-14.5+2.1 \log \mathrm{L}_{\star}
$$

Using the same methodology discussed in Section 2.2.3, Wachter et al. (2002) presented an improved mass-loss rate description for carbon-rich AGB stare. The dependence of the mass-loss rate on the pulsation period in the model has been accounted for by applying an observed period-luminosity relation. The resulting semi-empirical relation is

$$
\log \dot{M}=8.86+2.47 \log \mathrm{L}_{\star}-6.81 \log \mathrm{T}_{\mathrm{eff}}-1.95 \log \mathrm{M}_{\star} .
$$

In 2005, van Loon et al. (2005) analysed the optical spectra and infrared photometric data of a sample of dust-enshrouded RSG and O-rich AGB stars in the LMC to derive an empirical mass-loss rate formula, under the assumption of a gas-to-dust ratio, $\psi=$ $\rho_{\text {gas }} / \rho_{\text {dust }}$, of 500 :

$$
\log \dot{M}_{\mathrm{vL} 05}=12.478+1.05 \log \mathrm{L}_{\star}-6.3 \log \mathrm{T}_{\mathrm{eff}} .
$$

In the same year, Bergeat \& Chevallier (2005) derived an empirical mass-loss rate formula for carbon-rich AGB stars by extracting the mass-loss rate, expansion velocities, and dustto-gas density ratios from millimeter observations reported in the literature. Distances and luminosities previously estimated from Hipparcos data, masses from pulsations, $\mathrm{C} / \mathrm{O}$ abundance ratios from spectroscopy, and effective temperatures from a new homogeneous scale were used. The derived empirical relation is dependent on the effective temperature:

$$
\begin{array}{lcc}
\log \dot{M}_{\mathrm{B} 05}=24.93-9.20 \log \mathrm{T}_{\text {eff }} & \left(\mathrm{T}_{\text {eff }}<2400 \mathrm{~K}\right) & 36 . \\
\log \dot{M}_{\mathrm{B} 05}=-3.6-0.8 \log \mathrm{T}_{\text {eff }} & \left(2400 \mathrm{~K}<\mathrm{T}_{\text {eff }}<2900 \mathrm{~K}\right) & 37 . \\
\log \dot{M}_{\mathrm{B} 05}=20.65-7.83 \log \mathrm{T}_{\text {eff }} & \left(2900 \mathrm{~K}<\mathrm{T}_{\text {eff }}\right) & 38
\end{array}
$$

Srinivasan et al. (2009) used Spitzer Space Telescope data to relate the excess emission at 8 and $24 \mu \mathrm{m}$ from evolved AGB stars in the LMC to their mass-loss rate. The derived empirical relations are

$$
\begin{array}{ll}
\log \dot{M}_{\mathrm{S} 09}=-14.2+1.7 \log \mathrm{L}_{\star} & (\mathrm{C} / \mathrm{O}<1) \\
\log \dot{M}_{\mathrm{S} 09}=-14.96+1.87 \log \mathrm{L}_{\star} & (\mathrm{C} / \mathrm{O}>1) .
\end{array}
$$


Goldman et al. (2017) used a survey of $1612 \mathrm{MHz}$ OH maser observations of AGB and RSG stars in the LMC. This sample of sub-solar metallicity OH/IR stars was supplemented with data from the galactic centre and galactic bulge to derive a new empirical mass-loss rate prescription which depends on luminosity, pulsation period, and gas-to-dust ratio $\psi$ :

$$
\log \dot{M}_{\mathrm{G} 17}=-10.6+0.9 \log \mathrm{L}_{\star}+0.75 \log P-0.03 \log (\psi / 200) .
$$

By neglecting the weak dependence on the gas-to-dust ratio and adopting the same periodradius-mass relation as Vassiliadis \& Wood (1993), Eq. 41, turns into

$$
\log \dot{M}_{\mathrm{G} 17}=-1.28+1.62 \log \mathrm{L}_{\star}-2.91 \log \mathrm{T}_{\mathrm{eff}}-0.675 \log \mathrm{M}_{\star} .
$$

Lately, Beasor et al. (2020) measured the mass-loss rate and luminosities for RSG stars that reside in two clusters where the age and initial mass, $M_{\text {ini }}$, are known. By combining the results with those of clusters with a range of ages, mass-loss rate prescriptions are derived that show an explicit dependence on the initial mass

$$
\log \dot{M}_{\mathrm{B} 20}=\left(-26.4-0.23 M_{\text {ini }}\right)+4.8 \log \mathrm{L}_{\star} .
$$

Recently, Kee et al. (2020) have studied the role of vigorous atmospheric turbulence in initiating and determining the mass-loss rate of RSG stars, following both an analytical and numerical approach. They provide the first theoretical and fully analytic mass-loss rate prescription for RSG stars. They derived that

$$
\dot{M}_{\mathrm{K} 20}=\dot{M}_{\text {an }}\left(\frac{v_{\text {turb }} /\left(17 \mathrm{~km} \mathrm{~s}^{-1}\right)}{v_{\mathrm{esc}}\left(\mathrm{M}_{\star}, \mathrm{R}_{\star}\right) /\left(60 \mathrm{~km} \mathrm{~s}^{-1}\right)}\right)^{1.3},
$$

where $v_{\text {turb }}$ and $v_{\text {esc }}$ denote the turbulent and escape velocity, respectively. $\dot{M}_{\text {an }}$ is given by

$$
\dot{M}_{\text {an }}=4 \pi \rho\left(R_{\mathrm{p}, \bmod }\right) \sqrt{c_{s}^{2}+v_{\text {turb }}^{2}} R_{\mathrm{p}, \bmod }^{2},
$$

with $c_{s}$ the sound speed and $R_{\mathrm{p}, \text { mod }}$ the modified Parker radius

$$
R_{\mathrm{p}, \bmod }=\frac{G \mathrm{M}_{\star}\left(1-\Gamma_{\mathrm{Edd}}\right)}{2\left(c_{s}^{2}+v_{\mathrm{turb}}^{2}\right)},
$$

with $\Gamma_{\text {Edd }}$ the Eddington factor, $\Gamma_{\text {Edd }}=\kappa \mathrm{L}_{\star} /\left(4 \pi G \mathrm{M}_{\star} c\right)$, and $\kappa$ the flux weighted mean opacity taken to be $0.01 \mathrm{~cm}^{2} \mathrm{~g}^{-1}$. The density at the modified Parker radius, $\rho\left(R_{\mathrm{p}, \text { mod }}\right)$, is computed from hydrostatic stratification up to $R_{\mathrm{p}, \bmod }$ so that

$$
\rho\left(R_{\mathrm{p}, \bmod }\right)=\frac{4}{3} \frac{R_{\mathrm{p}, \bmod }}{\kappa R_{\star}^{2}} \frac{\exp \left[-\frac{2 R_{\mathrm{p}, \mathrm{mod}}}{R_{\star}}+\frac{3}{2}\right]}{1-\exp \left[-\frac{2 R_{\mathrm{p}, \bmod }}{R_{\star}}\right]} .
$$

We here use a turbulent velocity of $18.2 \mathrm{~km} \mathrm{~s}^{-1}$, which is the mean turbulent velocity required for their theoretical model to reproduce observationally inferred gas mass-loss rates.

Each of the above mass-loss rate prescriptions shows an explicit dependence on the luminosity, $\dot{M} \propto \mathrm{L}_{\star}{ }^{\alpha_{L}}$, with regression coefficient $\alpha_{L}$ ranging between $0.9-4.8$. For AGB stars, the semi-empirical formulae of Blöcker (1995) and Vassiliadis \& Wood (1993) have the steepest dependence on $\mathrm{L}_{\star}$; in the first case $\alpha_{L}=3.7$ and in the latter case the mass-loss 
rate is exponentially dependent on $\mathrm{L}_{\star}$. The recently derived RSG mass-loss rate prescription by Beasor et al. (2020) displays a very steep dependence on the luminosity of $\alpha_{L}=4.8$ when compared with those of de Jager et al. (1988) and Nieuwenhuijzen \& de Jager (1990) which are also valid for RSG stars.

To illustrate the range of AGB mass-loss rates and their dependence on the luminosity, a set of fundamental stellar parameters was calculated for values of the hydrogen-depleted core mass, $M_{c}$, ranging between $0.56-1.4 \mathrm{M}_{\odot}$. The luminosity was calculated using the Paczysnki core-mass-luminosity (CML) relation (Paczyński 1970):

$$
\mathrm{L}_{\star}=59250\left(M_{c}-0.522\right)
$$

with both quantities in solar units. Paczynski's relation is valid for $M_{c}>0.57 \mathrm{M}_{\odot}$. Over the years, continual upgrades in the input physics and more detailed stellar evolution calculations resulted in various other CML relations being proposed (see, e.g. Groenewegen \& Marigo 2004). For values of $M_{c}<0.68 \mathrm{M}_{\odot}$, we have opted to rely an the CML relation derived by Wagenhuber \& Groenewegen (1998), where the core mass limit is chosen to guarantee a smooth transition between both CML relations. For a fixed effective temperature or stellar mass, the other fundamental parameter can be determined from the Iben (1984) radius-luminosity-mass relation for evolving AGB stars

$$
\log \mathrm{R}_{\star}=\log (312)+0.68 \log \left(\frac{\mathrm{L}_{\star}}{10^{4}}\right)-0.31 \cdot \mathcal{S} \log \left(\frac{\mathrm{M}_{\star}}{1.175}\right)+0.088 \log \left(\frac{Z}{0.001}\right)-0.52 \log \left(\frac{l}{H_{p}}\right),
$$

with $Z$ the initial mass abundance of elements heavier than helium, $l / H_{p}$ the ratio of mixing length to pressure scale height, and the dimensionless parameter $\mathcal{S}=0$ for $\mathrm{M}_{\star} \leq 1.175 \mathrm{M}_{\odot}$ and $\mathcal{S}=1$ otherwise. We here take $Z=0.02$ and assume $l / H_{p}=1$. For a stellar mass of $2 \mathrm{M}_{\odot}$ or an effective temperature of $2800 \mathrm{~K}$, the various mass-loss rate relations discussed here are shown in the upper panels of Figure 5

For the RSG stars, we follow the procedure of Kee et al. (2020) for an effective temperature of $3500 \mathrm{~K}$. The radius is computed from the luminosity-radius-temperature relation $\mathrm{L}_{\star}=4 \pi \mathrm{R}_{\star}^{2} T_{\text {eff }}^{4}$. For the bottom right panel of Figure 5 in which only the effective temperature is fixed, the mass is computed from the mass-luminosity relation $\mathrm{M}_{\star}=(L / 15.5)^{1 / 3}$, following Kee et al. 2020).

The impact of a particular choice of mass-loss rate prescription on stellar evolution calculations is shown in Figure 6. A set of simplified evolutionary tracks has been constructed for stars with an AGB mass at the first thermal pulse of $(0.8,1.0,1.2,1.4,1.6,2.0$, $2.5,3) \mathrm{M}_{\odot}$. The corresponding core mass at the first thermal pulse was calculated using Eq. 13 of Wagenhuber \& Groenewegen (1998), and the luminosity was derived from the core-mass-luminosity (CML) relation described above. The stars were assumed to be Miratype variables with fundamental mode period given by Eq. 29. The core mass increases owing to nuclear burning where the rate is given by $\dot{M}_{c}=1.02 \times 10^{-11} \mathrm{~L}_{\star}$. The evolution of mass and luminosity for solar composition stars is shown in Figure 6 . The CML relation implies that $\mathrm{dL}_{\star} / \mathrm{d} t \propto \mathrm{d} M_{c} / \mathrm{d} t$, and since $\mathrm{L}_{\star} \propto$ the hydrogen fusion rate $\propto \mathrm{d} M_{c} / \mathrm{d} t$, the rate of change of the abscissa is $\mathrm{d} \log \mathrm{L}_{\star} / \mathrm{d} t \sim 59250 \cdot 1.02 \times 10^{-11} \mathrm{yr}^{-1}$ or $0.605 \mathrm{Myr}^{-1}$ (as indicated by the black arrow in the lower right panel of Figure 6. The remnant mass for the planetary nebula nucleus or white dwarf is indicated by the intersection of the evolutionary track with the CML relation. 


\subsection{Condensates identified in the winds of cool evolved stars}

Table 4 and Table 5 give more detailed information about the dust condensates identified in the winds of cool ageing stars, including references to the optical constants and to identifications from observations or presolar grains.

\subsection{Theory of dust nucleation}

Following the cluster model of nucleation, atoms and molecules undergo a sequence of association and recombination reactions to form polyatomic molecules of ever-increasing size, the gas-phase clusters (see Figure 7). In general, association reactions can be divived in two types - radiative association (which is difficult to detect in the laboratory) and collisional association (often 'association' is used as a shorthand) - that describe what happens to the collisional intermediate, $\mathrm{AB}^{\star}$ :

(1) $\mathrm{A}+\mathrm{B} \rightarrow \mathrm{AB}^{\star} \rightarrow \mathrm{AB}+$ photon - the intermediate either stabilises to $\mathrm{AB}$ by emitting a photon, or returns to reactants $\mathrm{A}$ and $\mathrm{B}$

(2) $\mathrm{A}+\mathrm{B} \rightarrow \mathrm{AB}^{\star}+\mathrm{M} \rightarrow \mathrm{AB}+\mathrm{M}$ - the intermediate stabilises through collision with a third body $\mathrm{M}$ or returns to reactants.

In order for reaction (2) to proceed to $A B$, the density of $M$ must be high, around $10^{13} \mathrm{~cm}^{-3}$, typically, so this process is usually negligible in the ISM. At densities lower than $\sim 10^{13} \mathrm{~cm}^{-3}$, reaction (1) can occur but whether it does efficiently depends on whether the lifetime of the complex $\mathrm{AB}^{\star}$ is longer than the radiative time scale. If so, then the complex can emit a photon and stabilise, if not it falls back to reactants. Generally, the larger the complex and the lower the temperature, the longer it lives and the more chance it has to emit a photon.

Dust formation occurs at high density and temperature under conditions under which one would expect collisional association reactions to be feasible. They may dominate over normal two-body reactions at high density. In this regard, collisional association reactions are acting essentially like three-body reactions.

In what follows, we consider both the kinetic theory and the classical thermodynamic theory of nucleation to describe the nucleation process. This section is mainly based on Patzer et al. (1998), Gail \& Sedlmayr (2013), and Mauney et al. (2015).

7.3.1. Kinetic nucleation theory. The master equation for the particle number density $n(N, t)$ of a cluster of size $N$ is

$$
\frac{\mathrm{d} n(N, t)}{\mathrm{d} t}=\sum_{i=1}^{I} J_{i}^{c}(N, t)-\sum_{i=1}^{I} J_{i}^{c}(N+i, t)
$$

where $i$ denotes the $i$ th geometrical configuration of a molecule (henceforth called $i$-mer), $I$ the maximum molecular $i$-mer contributing, and $J_{i}^{c}(N, t)$ the effective flux (or transition rate) for the growth of the particle of size $N-i$ to size $N$. The effective transition rate is given by

$$
J_{i}^{c}(N, t)=\sum_{r_{i}-1}^{R_{i}}\left(\frac{n(N-i, t)}{\tau_{\mathrm{gr}}\left(r_{i}, N-i, t\right)}-\frac{n(N, t)}{\tau_{\mathrm{ev}}\left(r_{i}, N, t\right)}\right)
$$


Table 4 Condensates identified in the winds of cool evolved stars using infrared data

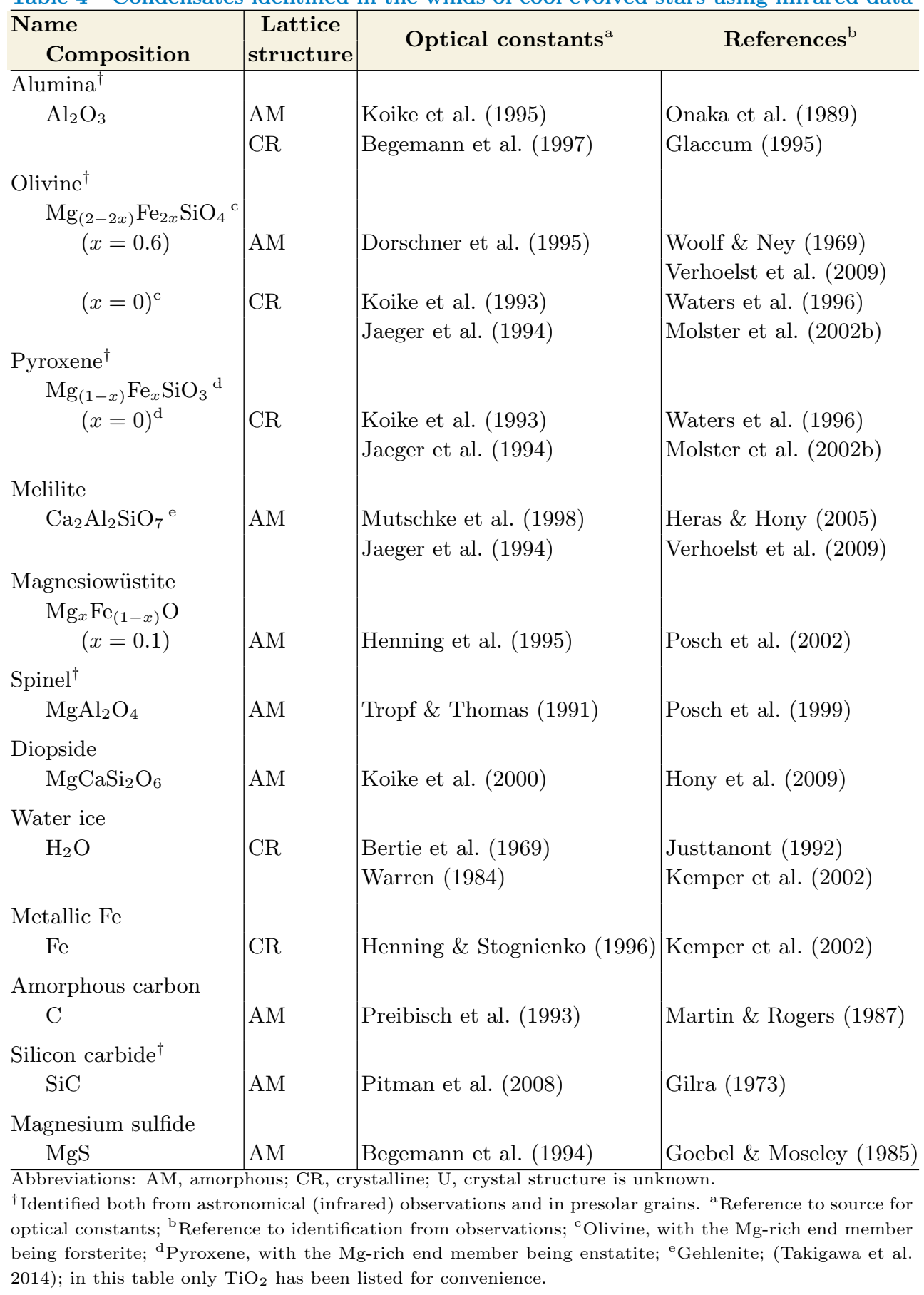


Table 5 Condensates only identified in presolar grains with AGB/RSG origin

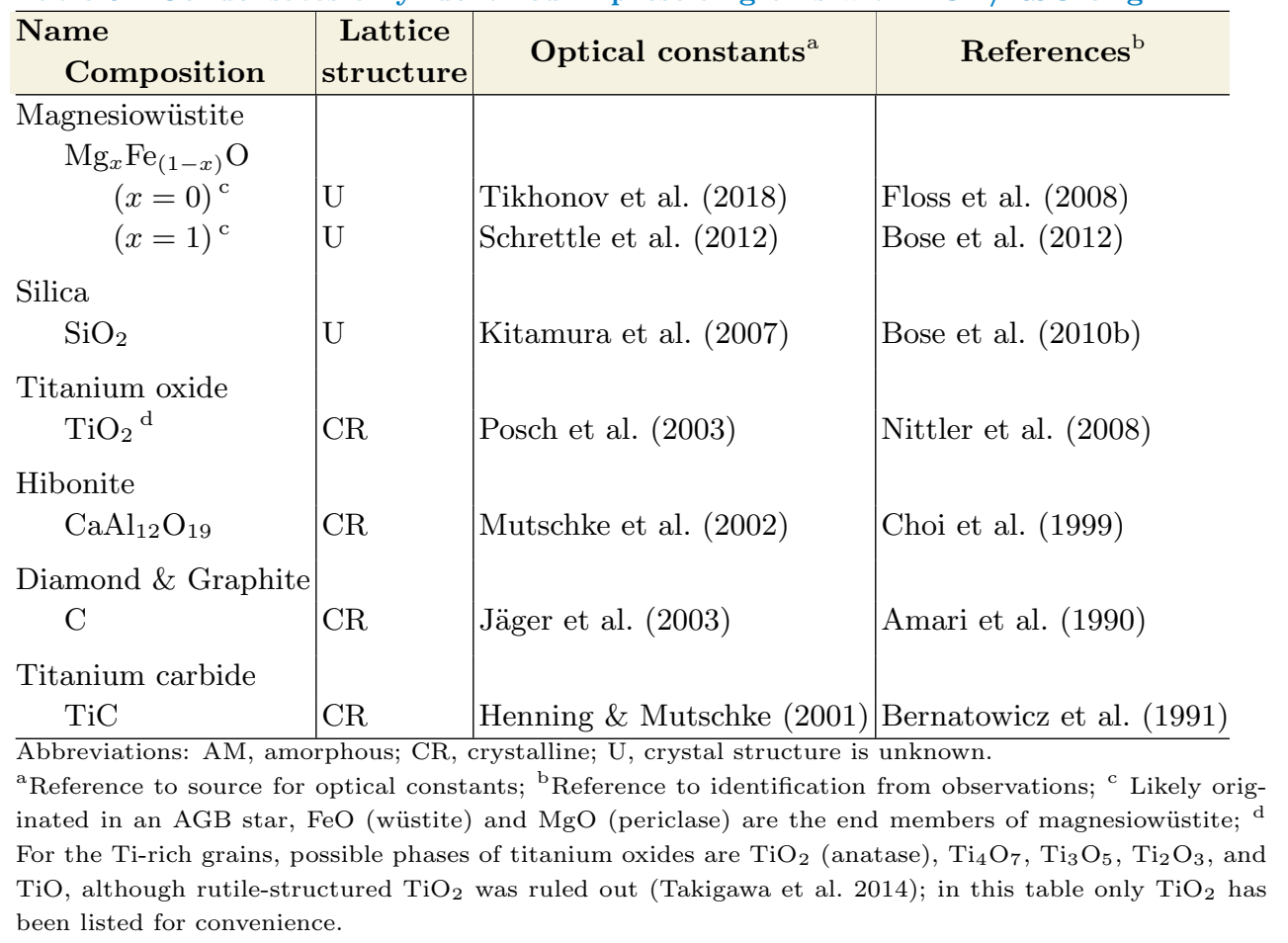

with $r_{i}$ denotes a chemical reaction in which the $i$-mer is involved. The time scale of the growth reaction from the cluster of size $N-i$ to size $N, \tau_{\mathrm{gr}}\left(r_{i}, N-i, t\right)$ is given by

$$
\tau_{\mathrm{gr}}^{-1}\left(r_{i}, N-i, t\right)=A(N-i) \alpha\left(r_{i}, N-i\right) v_{\mathrm{rel}}\left(n_{f}\left(r_{i}\right), N-i\right) n_{f}\left(r_{i}, t\right)
$$

and the evaporation time scale leading from size $N$ to size $N-i$ by

$$
\tau_{\mathrm{ev}}^{-1}\left(r_{i}, N, t\right)=A(N) \beta(r, N) v_{\mathrm{rel}}\left(n_{r}\left(r_{i}\right), N\right) n_{r}\left(r_{i}, t\right),
$$

where $A(N)$ is the surface area of the cluster of size $N, \alpha$ and $\beta$ the reaction coefficients for the forward and backward process for reaction $r_{i}$ respectively, $n_{f}\left(r_{i}\right)$ and $n_{r}\left(r_{i}\right)$ the number density of the molecule of the growth (forward) and the evaporation (reverse) process for reaction $r_{i}$, and $v_{\text {rel }}=\sqrt{k T / 2 \pi \mu} \approx \sqrt{k T / 2 \pi m_{1}}$ the average relative velocity between the molecule of mass $m_{1}$ and the cluster of mass $m_{c}$ with $\mu=1 /\left(1 / m_{1}+1 / m_{c}\right)$. The reaction rates are determined from chemical kinetic theory or laboratory measurements.

Homogeneous nucleation is the process in which a single kind of gas-phase species is involved, while heterogeneous nucleation implies the action of several gas-phase species. They are principally the same process, but from a theoretical point of view, homogeneous nucleation allows a simpler mathematical treatment of the process. In that case, Eq. 51, is described by one of the $r_{i}$ reactions.

If the reaction efficiencies were all known, the growth and evaporation time scales could be calculated directly from Eqs. 52,53 and the transition rate could be derived without recourse to thermodynamic arguments. However, the growth efficiencies are often uncertain 
and the evaporation coefficients $\beta$ are mostly unknown. Therefore, in order to describe the nucleation process under the conditions of chemical non-equilibrium in the gas-phase and of thermal non-equilibrium between the gas and the clusters or dust particle, methods have been develop based on kinetic nucleation theory and thermodynamic arguments.

One therefore needs to define a reference equilibrium state which is characterized by thermal equilibrium with equal temperatures of the solid, the clusters and the gas, by phase equilibrium between the monomers as well as the $i$-mers and the bulk solid, and by simultaneous chemical equilibrium in the gas phase, i.e. it is a state of local thermodynamic equilibrium (LTE). All quantities referring to the LTE-state will be denoted with an ${ }^{\circ}$, For the LTE-state, the internal temperature of the $N$-clusters, $T_{d}(N)$, is equal to the equilibrium temperature, $\stackrel{\circ}{T}=T_{d}(N)$. Local thermodynamic equilibrium between the gas phase and clusters implies that the principle of detailed balance holds for the growth processes and their respective reverse reactions, and thus that the effective transition rate equals zero for all cluster size, $J_{i}^{c}(N, t)=0$. It follows that in LTE

$$
\frac{\stackrel{\circ}{n}(N-i)}{\stackrel{\circ}{\mathrm{gr}}_{\mathrm{gr}}\left(r_{i}, N-i\right)}=\frac{\stackrel{\circ}{n}(N)}{\stackrel{\circ}{\mathrm{evv}}_{\mathrm{e}}\left(r_{i}, N\right)} .
$$

The equilibrium particle densities can be determined from the law of mass action

$$
\left(\frac{\stackrel{\circ}{n}(N-i) \stackrel{\circ}{n}_{f}\left(r_{i}\right)}{\stackrel{\circ}{n}(N) \stackrel{\circ}{n}_{r}\left(r_{i}\right)}\right)=\exp \left(\frac{\Delta_{r} G^{\ominus}\left(r_{i}, N, T_{d}(N)\right)}{R T_{d}(N)},\right)
$$

with $R$ the gas constant and where the Gibbs free energy of the reaction $\Delta_{r} G^{\ominus}\left(r_{i}, N, T_{d}(N)\right)$ is given by the standard molar Gibbs free energy of formation of all reaction participants at temperature $T_{d}(N)$ in the reference state ' $\theta$ '; see Eq. (7) in Patzer et al. (1998). These Gibbs free energies of formation are determined from (complex) chemical modelling.

In what follows, we will derive the stationary nucleation rate for a situation of a homogeneous, homomolecular process in thermal equilibrium. For a stationary flow, $\mathrm{d} n(N, t) / \mathrm{d} t=0$. From Eq. 50 it follows that the transition rate for clusters with $N \geq 2$ are equal in size space at time $t, J_{1}^{c}(N, t)=J_{1}^{c}(N+1, t) \equiv J_{\star}(t) . J_{\star}(t)$ is the size-independent rate of cluster formation. It then can be shown

$$
J_{\star}(t)=\left[\sum_{N=1}^{N_{\max }}\left(\frac{\tau_{\mathrm{gr}}\left(r_{1}, N, t\right)}{\stackrel{\circ}{n}(N)}\right)\right]^{-1},
$$

with

$$
\tau_{\mathrm{gr}}^{-1}\left(r_{1}, N, t\right)=A(N) \alpha(N) v_{\mathrm{rel}}(n(1), N) n(1, t) .
$$

Consequently, the task of calculation the rate of cluster formation implies the determination of the growth time scales, $\tau_{\mathrm{gr}}$, and the determination of the thermodynamic properties of the clusters from equilibrium thermodynamics.

For a process in thermal equilibrium with the gas and further assuming that the temperature of the cluster does not depend on size, $T_{d}(N)=T_{g}=T$, the equilibrium cluster size distribution can be expressed by a Boltzmann-like distribution

$$
\stackrel{\circ}{n}(N)=\stackrel{\circ}{n}(1) \exp \left(-\frac{\Delta G(N)}{R T}\right),
$$


with $\stackrel{\circ}{n}(1)$ the equilibrium distribution of the monomer corresponding to the vapour saturation pressure $p_{\text {sat }}(T) . \Delta G(N)$ denotes the free energy change associated with the formation of a cluster of size $N$ from the saturated vapour, which is related to the standard molar Gibbs free energy of formation of the $N$-cluster $\Delta_{f} G^{\ominus}(N)$ by

$$
\Delta G(N)=\Delta_{f} G^{\ominus}(N)+R T \ln \left(\frac{p_{\text {sat }}(T)}{p^{\ominus}}\right)-N \Delta_{f} G_{1}^{\ominus}(s),
$$

where $\Delta_{f} G_{1}^{\ominus}(s)$ is the standard molar Gibbs free energy of formation of the solid phase and $p^{\ominus}$ is the pressure of the standard state.

The time scale $\tau_{g r}\left(r_{1}, N, t\right)$ on the right hand size of Eq. 56] grows with increasing cluster size $N$ less than exponentially; for spherical grains it is $\propto N^{2 / 3}$. This implies that for sufficiently large $N$, the terms of the sum become negligible. The size $N_{\max }$ then denotes the maximum cluster size for which all $N$ above $N_{\max }$ do not contribute to the sum. Eq. 56 can be further simplified by realising that there exists some critical size $N_{\star}$ where $\stackrel{\circ}{n}$ attains its absolute minimum. The growth reaction of this cluster of size $N_{\star}$ is the bottleneck of the chemical network. It is energetically favourable for clusters of size $N=N_{\star}+1$ to continue to form larger clusters that eventually form a 'macroscopic' dust grain. We hence may approximate the whole sum by the largest terms, so that

$$
J_{\star}(t) \simeq \frac{\stackrel{\circ}{n}\left(N_{\star}\right)}{\tau_{\mathrm{gr}}\left(r_{1}, N_{\star}, t\right)}=\stackrel{\circ}{n}\left(N_{\star}\right) n(1, t) A\left(N_{\star}\right) \alpha\left(N_{\star}\right) v_{\mathrm{rel}} \equiv \stackrel{\circ}{n}\left(N_{\star}\right) f\left(N_{\star}, t\right),
$$

where $f\left(N_{\star}, t\right)$ is the monomer attachment rate and the reaction $r_{1}$ is the rate determined by the reaction

$$
M_{N_{\star}}+M_{1} \rightarrow M_{N_{\star}+1}
$$

In general, truncating the summation in Eq. 56, at $N_{\max }=2 N_{\star}$ has negligible effect on the result. Note that Eq. 61 inherently assumes that the addition of a monomer, $A_{1}$, is the rate limiting step. This implies that the ratio of association with the least abundant cluster along the chain of reactions determines the rate at which macroscopic particles form. For that reason, $J_{\star}$ is also called the rate of seed formation or the nucleation rate. For the example of $(\mathrm{SiO})_{n}$, the critical cluster size is $N_{\star}=4-6$ for temperatures slightly below 630 K (Gail \& Sedlmayr 2013, Bromley et al. 2016) .

7.3.2. Classical nucleation theory. For species for which no thermochemical data are available, one often resorts to classical nucleation theory (CNT), an effective theoretical tool originally developed in the early 20 th century and based on the concepts of classical continuum theory. It refers to a state of constrained equilibrium between the clusters and the supersaturated vapor at the same supersaturation ratio $S$ and temperature $T$. Although widely used in the astronomical community, it can only be applied if (1) $\Delta G(N)$ can be approximated by macroscopic parameters such as the surface tension of the bulk solid, (2) the cluster are in thermal equilibrium, (3) small clusters are present in equilibrium concentrations, and (4) chemical equilibrium prevails in the gas phase.

CNT uses the capillary approximation to represent the potentially unknown $\Delta G(N)$ via the surface tension of the bulk material, $\sigma_{\infty}$, as measured in the laboratory:

$$
\frac{\Delta G(N)}{R T}=-N \ln (S)+\sigma_{\infty} A(N)
$$


Assuming the monomers to be small spheres of radius $a_{1}, A(N)=4 \pi a_{1}^{2}$ and we can write that

$$
\frac{4}{3} \pi a_{1}^{3}=N V_{1}
$$

with $V_{1}$ the volume corresponding to one monomer in the bulk solid. Under this assumption

$$
\frac{\Delta G(N)}{R T}=-N \ln (S)+\sigma_{\infty}(36 \pi)^{1 / 3} N^{2 / 3} V_{1}^{2 / 3}
$$

or

$$
\frac{\Delta G(N)}{R T}=-N \ln (S)+\theta_{\infty} N_{\star}^{2 / 3} \text { with } \theta_{\infty}=\frac{4 \pi a_{1}^{2} \sigma_{\infty}}{R T} .
$$

Clearly, the capillary approximation is inadequate for small values of $N$ and where a spherical approximation of the cluster geometry is poor. Moreover, it does not yield the correct limit $\Delta G(N) \rightarrow 0$ for $N \rightarrow 1$. Therefore, a modification has been proposed to allow for a consistent formulation

$$
\frac{\Delta G(N)}{R T}=-(N-1) \ln (S)+\theta_{\infty}\left(N_{\star}^{2 / 3}-1\right) .
$$

Since CNT refers to a constrained equilibrium, the equilibrium particle density of the monomer in this state is equal to the monomer particle density in the actual situation, $\stackrel{\circ}{n}(1)=n(1, t)$. For a homogeneous and homomolecular process, the CNT stationary nucleation rate for the critical cluster of size $N_{\star}$ is given by

$$
J_{\star}(t)=z\left(N_{\star}\right) \gamma A\left(N_{\star}\right)(n(1, t))^{2} v_{\text {rel }} \exp \left(-\Delta G_{\star} / k T\right),
$$

67.

where $z$ is the Zel'dovich factor (see below), $\gamma$ the sticking coefficient (the probability that an incoming monomer binds to the target cluster, often assumed to be unity in CNT) and $\Delta G_{\star}=\Delta G\left(N_{\star}\right)$. Using Eq. 58 and writing the monomer attachment rate for a cluster of size $N$ as

$$
f(N, t)=\gamma A(N) n(1, t) v_{\mathrm{rel}},
$$

Eq. 67. can be written in a more concise way

$$
J_{\star}(t)=z\left(N_{\star}\right) f\left(N_{\star}, t\right) \stackrel{\circ}{n}\left(N_{\star}\right),
$$

hence resembling Eq. 60, except for the correction factor $z$. The kinetic Zel'dovich factor $z$ accounts for the loss of nuclei during their Brownian motion, and in the classical theory it takes the form of

$$
z\left(N_{\star}\right)=\left(\frac{1}{2 \pi R T}\left|\frac{\partial^{2} \Delta G(N)}{\partial N^{2}}\right|_{N_{\star}}\right)^{1 / 2} .
$$

It is straightforward to derive that

$$
z\left(N_{\star}\right)=\left(\left(\frac{2 V_{1}}{9 \pi}\right)^{2 / 3} \frac{\sigma_{\infty}}{R T} \frac{1}{N_{\star}^{4 / 3}}\right)^{1 / 2}
$$

The minimum value of $\stackrel{\circ}{n}(N)$ is encountered where $\partial \Delta G / \partial N=0$; the corresponding value of $N$ (i.e., the critical cluster size $N_{\star}$ ) is then

$$
N_{\star}=\left(\frac{8 \pi}{3}\left(\frac{3 V_{1}}{4 \pi}\right)^{2 / 3} \frac{\sigma_{\infty}}{\ln S}\right)^{3} .
$$


The critical size of refractory grains is usually small owing to the large temperatures at which nucleation occurs. This implies that the capillary approximation can destroy the accuracy of the results since the nucleation rate depends exponentially on the energy of cluster formation, which in this case, is only poorly approximated via the surface tension of the bulk.

7.3.3. Modified classical nucleation theory. Aiming to improve CNT, modified classical nucleation theory (MCNT) links the macroscopic solid bulk and microscopic cluster properties by accounting for the curvature on the surface for small clusters. As a first step, one obtains the thermochemical properties of some small clusters, for example via density functional theory (DFT) and the calculation of the Gibbs free energy. This input is then used to approximate the bulk surface tension, $\sigma_{\infty}$, by fitting the following equation

$$
\frac{\Delta G(N)}{k T}=\theta_{\infty} \frac{N-1}{(N-1)^{1 / 3}+N_{f}^{1 / 3}} .
$$

to the available data. The parameter $N_{f}$ is a fitting factor defined as the (integer) monomer cluster size where the surface energy is reduced to half of the bulk value, or is left as a free parameter in the fitting procedure (see, for example, Fig. 2 in Lee et al. 2015). Eq. 73 can then be used to calculate the Gibbs free energies of formation and partial pressures for arbitrary values of $N$. The Zel'dovich factor then reduces to

$$
z\left(N_{\star}\right)=\left(\frac{\theta_{\infty}}{9 \pi\left(N_{\star}-1\right)^{4 / 3}} \frac{1+2\left(\frac{N_{f}}{N_{\star}-1}\right)^{1 / 3}}{\left(1+\left(\frac{N_{f}}{N_{\star}-1}\right)^{1 / 3}\right)^{3}}\right)^{1 / 2} .
$$

where the critical cluster size $N_{\star}$ is

$$
N_{*}-1=\frac{N_{*, \infty}}{8}\left(1+\sqrt{1+2\left(\frac{N_{f}}{N_{*, \infty}}\right)^{1 / 3}}-2\left(\frac{N_{f}}{N_{*, \infty}}\right)^{1 / 3}\right)^{3},
$$

and

$$
N_{*, \infty}=\left(\frac{\frac{2}{3} \theta_{\infty}}{\ln S(T)}\right)^{3}
$$

7.3.4. Atomistic classical nucleation theory. The capillary approximation in CNT offers a reasonable description of nucleation when $n \gg 1$. In the case of $n \rightarrow 1$, the more exact atomistic model is required. One therefore constructs the free energy of a cluster of size $N$ as

$$
G(N)=G_{V}(N)+G_{S}(N),
$$

where $G_{V}(N)$ is referred to as the volume term, $G_{V}(N)=-N k T \ln (S)$, and $G_{S}(N)$ as the surface term. In the atomistic case, one uses

$$
G_{\mathrm{S}}(N)=\lambda N-E_{b}(N)
$$

where $E_{b}(N)$ is the binding energy of a cluster of size $N$ and $\lambda N$ represents the binding energy of $N$ monomers in the bulk solid phase with bulk cohesive energy $\lambda$. The binding energy is given by

$$
E_{b}(N)=N E_{1}-E_{t}
$$




\section{Nucleation rates from models}

The latest radiation hydrodynamical models for O-rich winds of Höfner et al. (2016) indicate that the fractional seed particle abundance, $n_{d} / n_{\mathrm{H}}$, should be above $\sim 10^{-16}$ for a dust-driven wind of mass-loss rate $1 \times 10^{-7} \mathrm{M}_{\odot} \mathrm{yr}^{-1}$ to be initiated. Winds of mass-loss rate $7.8 \times 10^{-5} \mathrm{M}_{\odot} \mathrm{yr}^{-1}$ are generated for $n_{d} / n_{\mathrm{H}}=10^{-15}$ (Bladh et al. 2019). After further grain growth, the final $n_{d} / n_{\mathrm{H}}$ ratio is around $10^{-12}-10^{-13}$ for C-rich winds (see Figure 4). With a typical AGB pulsation period ranging between $10^{7}-10^{8}$ sec, this translates into a stationary nucleation rate $J_{\star} / n_{\mathrm{H}} \approx n_{d} /\left(n_{\mathrm{H}} \Delta t\right)$ of $\sim 10^{-19}-10^{-24} \mathrm{~s}^{-1}$.

with $E_{t}$ the total energy and $E_{1}$ the single monomer total energy. The difficulty lies in determining $E_{b}(N)$ which - similar to the kinetic description - is the result of complex chemical and quantum mechanical interactions within the molecule and requires DFT calculations. If DFT calculations are available, the number densities are calculated as

$$
n(N)=n(1) \exp \left(-\frac{G(N)}{k T}\right)
$$

and Eq.69] yields the stationary nucleation rate. Recourse to the kinetic formulation, shows that the Zel'dovich factor then can be given in terms of $n(N)$ :

$$
z\left(N_{\star}\right)=\left(\sum_{N=1}^{N_{\max }} \frac{n\left(N_{\star}\right)}{n(N)}\right)^{-1}
$$

where $N_{\max }>N_{\star}$ is a reasonable cut-off of the summation. The atomistic formulation of CNT (ACNT) hence also accounts for clusters with irregular shapes.

7.3.5. Comparing the various levels of theory. Starting at the lowest level of complexity, it first should be noted that publications are not always clear as to whether CNT or MCNT was used as the modelling approach and hence if the bulk or a fictitious surface tension was used to represent the Gibbs free energies. Aiming to unravel the role of various nucleation candidates in O-rich AGB winds, Jeong et al. (2003) have pursued a mixed approach with $\mathrm{TiO}_{2}$ studied at the level of MCNT based on Jeong et al. (2000), and $\mathrm{Fe}, \mathrm{SiO}_{2}, \mathrm{SiO}$, $\mathrm{TiO}$, and $\mathrm{Al}_{2} \mathrm{O}_{3}$ at the level of CNT. Of the candidate materials considered, only $\mathrm{TiO}$ and $\mathrm{TiO}_{2}$ reach nucleation rates greater than $\sim 10^{-24} \mathrm{~s}^{-1}$ for the typical pressures in the dust condensation region of $10^{-3}-10^{-1} \mathrm{dyn} \mathrm{cm}^{-2}$ (Höfner et al. 2016). (See the sidebar titled Nucleation rates from models.) A problem, however, is that the kinetic temperature should be lower than $\sim 1200 \mathrm{~K}$ for the $\mathrm{TiO} / \mathrm{TiO}_{2}$ nucleation to start, which is below the typical kinetic temperatures of $\sim 1500-1800 \mathrm{~K}$ in the dust formation region.

Lee et al. (2018) used the small cluster thermochemical data of $\left(\mathrm{TiO}_{2}\right)_{N}(N=1-10)$ to study the stationary nucleation rate following both a MCNT and KNT approach. For regions where the supersaturation ratio $S \gg 1$ both results agree within one order of magnitude. However, as the values of the gas temperature and pressure reach the $S=1$ equilibrium zone, larger differences between both methods occur, reaching four orders of magnitude. This difference is caused by limitations in both MCNT and KNT. For $S \rightarrow 1$, KNT reaches its limits since the critical cluster size $N_{\star}$ increases rapidly in this regime. By truncating 
Eq. 56] at $N=10$, an unphysical KNT nucleation rate can be produced in regions of $S \lesssim 1$. This argues for further computational chemistry and experimentally based investigations into the small cluster properties for sufficiently large $N$.

Mauney et al. (2015) computed the thermochemical properties of $(\mathrm{C})_{N}(N=1-99)$ and used that input to compare CNT, ACNT, and KNT stationary nucleation rates. By considering large cluster geometries, they were able to capture $N_{\star}$ across a wide range of temperatures and pressures for carbon nucleation. The ACNT and KNT rates agree to very high order. This is a particular useful result for numerical modelling since the prescription of ACNT is much faster than KNT. However, compared to KNT (and hence ACNT), the CNT rates are much faster at temperatures relevant for dust nucleation in stellar winds. For low values of the supersaturation ratio, a difference of 60 orders of magnitude is deduced due to the much larger critical size in CNT. For values of the supersaturation rate above $\sim 7$ the CNT and KNT approach tend to agree.

Trying to understand under which conditions $(\mathrm{SiO})_{N}$ nucleation occurs, Bromley et al. (2016) followed a bottom-up kinetic modelling approach and have compared the ACNT and KNT stationary nucleation rates and performed a full time-resolved kinetic model. For

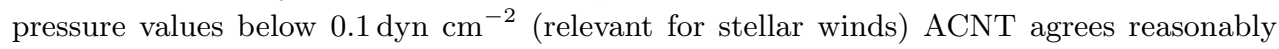
well with KNT, but overestimates $J_{\star}$ by up to a factor 1000 for higher pressure regions. The KNT stationary and full kinetic model agree well between $300-620 \mathrm{~K}$ for pressures

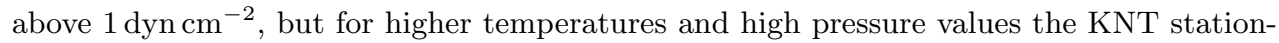
ary approach predicts higher nucleation rates. This difference arises because the KNT stationary approach assumes chemical steady-state which is less valid at higher temperatures where the dissociation rates of some $(\mathrm{SiO})_{N}$ clusters become very fast. Independent of the model approach, the kinetics of $\mathrm{SiO}$ are much too slow to explain dust formation above $1000 \mathrm{~K}$ under the conditions of stellar outflows. Goumans \& Bromley (2012) followed a KNT approach to study the heteromolecular nucleation of $\mathrm{Mg}, \mathrm{SiO}$, and $\mathrm{H}_{2} \mathrm{O}$ to form silicate clusters with an enstatite stoichiometry $\left(\mathrm{MgSiO}_{3}\right)_{2}$, but found the nucleation to be

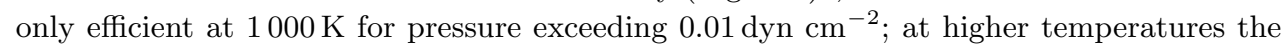
small clusters become thermodynamically unstable.

Supersaturation ratio: defined as the ratio $p_{1} / p_{\text {vap }}$ with $p_{1}$ the partial pressure of the monomers and $p_{\text {vap }}$ the total vapour pressure. This definition is only useful for homogeneous nucleation. 